\title{
Terahertz Ultra-Massive MIMO-Based Aeronautical Communications in Space-Air-Ground Integrated Networks
}

\author{
Anwen Liao, Zhen Gao, Member, IEEE, Dongming Wang, Member, IEEE, Hua Wang, Hao Yin, \\ Derrick Wing Kwan Ng, Fellow, IEEE, and Mohamed-Slim Alouini, Fellow, IEEE
}

\begin{abstract}
The emerging space-air-ground integrated network has attracted intensive research and necessitates reliable and efficient aeronautical communications. This paper investigates terahertz Ultra-Massive (UM)-MIMO-based aeronautical communications and proposes an effective channel estimation and tracking scheme, which can solve the performance degradation problem caused by the unique triple delay-beam-Doppler squint effects of aeronautical terahertz UM-MIMO channels. Specifically, based on the rough angle estimates acquired from navigation information, an initial aeronautical link is established, where the delay-beam squint at transceiver can be significantly mitigated by employing a Grouping True-Time Delay Unit (GTTDU) module (e.g., the designed Rotman lens-based GTTDU module). According to the proposed prior-aided iterative angle estimation algorithm, azimuth/elevation angles can be estimated, and these angles are adopted to achieve precise beam-alignment and refine GTTDU module for further eliminating delay-beam squint. Doppler shifts can be subsequently estimated using the proposed prior-aided iterative Doppler shift estimation algorithm. On this basis, path delays and channel gains can be estimated accurately, where the Doppler squint can be effectively attenuated via compensation process. For data transmission, a data-aided decision-directed based channel tracking algorithm is developed to track the beam-aligned effective channels. When the dataaided channel tracking is invalid, angles will be re-estimated at the pilot-aided channel tracking stage with an equivalent sparse digital array, where angle ambiguity can be resolved based on the previously estimated angles. The simulation results and the derived Cramér-Rao lower bounds verify the effectiveness of our solution.
\end{abstract}

Index Terms-Terahertz communications, aeronautical communications, ultra-massive MIMO, channel estimation and tracking, space-air-ground integrated network.

The codes and some other associated materials of this work may be available at https://gaozhen16.github.io.

A. Liao, Z. Gao, and H. Wang are with School of Information and Electronics, Beijing Institute of Technology, Beijing 100081, China (E-mails: \{liaoanwen, gaozhen16,wanghua\}@ bit.edu.cn).

D. Wang is with the National Mobile Communications Research Laboratory, Southeast University, Nanjing 210096, China (e-mail: wangdm@seu.edu.cn).

H. Yin is with Institute of China Electronic System Engineering Corporation, Beijing 100141, China (E-mail: yinhao@cashq.ac.cn).

D. W. K. Ng is with the School of Electrical Engineering and Telecommunications, University of New South Wales, Sydney, NSW 2052, Australia (e-mail: w.k.ng@unsw.edu.au).

M.-S. Alouini is with the Electrical Engineering Program, Division of Physical Sciences and Engineering, King Abdullah University of Science and Technology, Thuwal, Makkah Province, Saudi Arabia (E-mail: slim.alouini@kaust.edu.sa).

\section{INTRODUCTION}

Terahertz (THz) communication is expected to play a pivotal role in the future Sixth Generation (6G) wireless systems, which promise to provide ubiquitous connectivity with broader and deeper coverage [1]. THz-band (spectrum ranges from 0.1 to $10 \mathrm{THz}$ ) is envisioned to offer significantly larger bandwidths than millimeter-Wave (mmWave) for supporting up to tens of Gigahertz $(\mathrm{GHz})$ ultra-broadband and Terabit per second (Tbps) ultra-high peak data rate [2]-[4]. Meanwhile, $\mathrm{THz}$ communications can be conducive to realize the UltraMassive Multiple-Input Multiple-Output (UM-MIMO)-based transceivers equipped with tens of thousands of antennas (even the Uniform Planar Array (UPA) with size of $1024 \times 1024$ [5]), which can effectively combat the severe path loss of $\mathrm{THz}$ signals and further extend the communication range using beamforming techniques [6]-[8]. Therefore, THz UM-MIMO technique has been emerging as a promising candidate for the $6 \mathrm{G}$ mobile communication systems [1]. However, due to the severe atmospheric molecular absorption (such as water vapor) and rain attenuation [8], [9], the applications of $\mathrm{THz}$ communications are restricted to short-link distance [10][12]. Fortunately, those atmospheric molecule absorption and rain attenuation mainly occur in the troposphere, and these negative factors can be largely mitigated due to the negligible absorption in the stratosphere and above [13]-[15].

On the other hand, the ambitious $6 \mathrm{G}$ is poised to seamlessly integrate space-air networks with terrestrial mobile cellular networks. Against this background, the concept of Space-AirGround Integrated Network (SAGIN) is conceived and has attracted intensive research [16], [17]. As shown in Fig. 1, a typical SAGIN consists of three layers including spaceborne, airborne, and terrestrial networks [17]. The Geostationary Earth Orbit (GEO), Medium Earth Orbit (MEO), and Low Earth Orbit (LEO) satellites that operate at different altitudes constitute the spaceborne network. In the airborne network, aerial Base Stations (BSs) such as balloons and airships can jointly serve various aircrafts and Unmanned Aerial Vehicles (UAVs). In particular, numerous LEO satellites, aerial BSs, aircrafts, and UAVs can constitute the aeronautical ad hoc network to achieve the goal of "Internet above the clouds" [18], [19], which necessitates THz UM-MIMO technique to support 


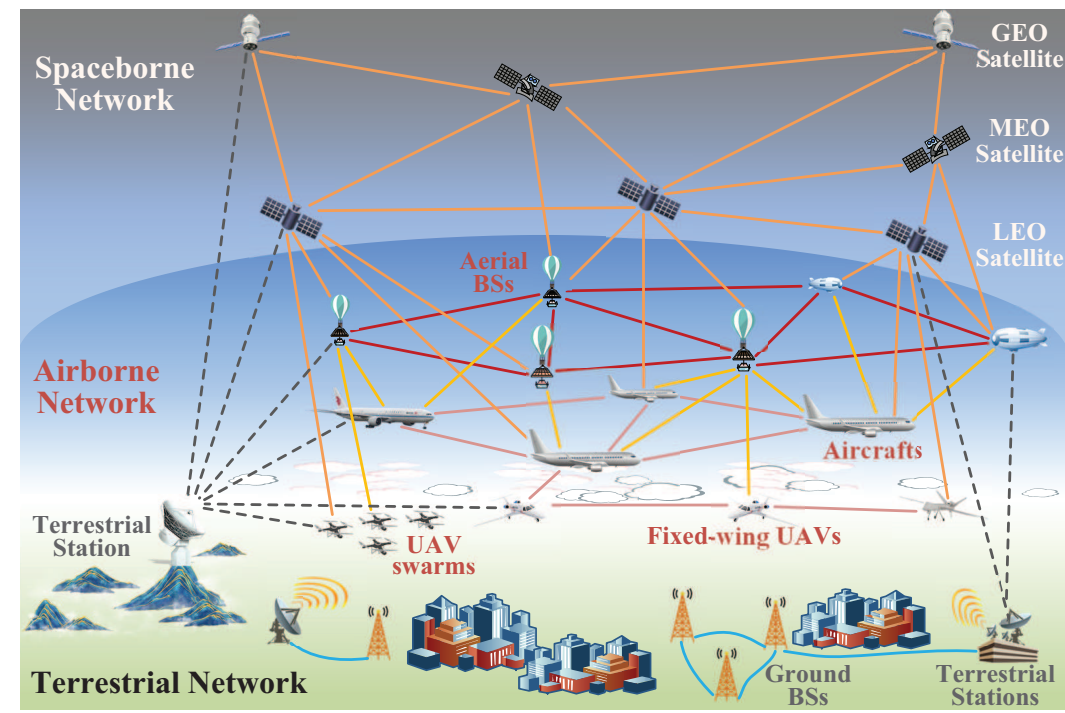

Fig. 1. Typical SAGIN includes spaceborne, airborne, and terrestrial networks, where numerous LEO satellites, aerial BSs, aircrafts, and UAVs together constitute the aeronautical ad hoc network [17], [19].

the reliable and efficient aeronautical communications ${ }^{1}$.

To guarantee the Quality-of-Service (QoS) for THz UMMIMO-based aeronautical communications, reliable Channel State Information (CSI) acquisition at the transceiver is indispensable [20]. However, due to the high-speed mobility of flying aircrafts/UAVs and the wobbles of aerial BSs, these aerial communication links exhibit the dramatically fast timevarying fading characteristics, which make accurate channel estimation and tracking rather challenging. To acquire the accurate estimate of fast time-varying channel, some channel estimation and tracking schemes [21]-[23] were proposed to reduce the training overhead caused by frequent channel estimation. In [21], a data-aided channel tracking scheme is proposed to estimate and track the partial channel coefficients of angle domain channels using lens antenna array. By exploiting the sparsity of the virtual channel vector in angle domain, the virtual channel parameters based on first order auto regressive model were estimated and tracked using the expectation maximization-based sparse Bayesian learning framework in [22], [23]. Moreover, by acquiring the dominant channel parameters including the Angle of Arrivals/Departures (AoAs/AoDs), Doppler shifts, and channel gains, rather than the complete MIMO channel matrix, some multi-stage channel estimation solutions were proposed in [24], [25] enabling fast channel tracking for narrow-band mmWave MIMO systems. Note that these schemes above just consider the channel estimation and tracking for common mmWave systems. In [26], a priori-aided $\mathrm{THz}$ channel tracking scheme with low pilot overhead was proposed to predict and track the physical direction of Line-of-Sight (LoS) component of the timevarying massive MIMO channels in THz beamspace domain. For the dynamic indoor short-range $\mathrm{THz}$ communications, the

\footnotetext{
${ }^{1}$ In general, civil aircrafts spend most of their flight time at the bottom of the stratosphere, where the relatively stable flight state is convenient for the establishment of $\mathrm{THz}$ communication links. Therefore, the aeronautical communications studied in this paper can be mainly aimed at the aircrafts flighted at the stratospheric.
}

authors in [27] proposed an AoA estimation method based on Markov process and Bayesian inference, where the forwardbackward algorithm is implemented to carry out the Bayesian inference.

However, the aforementioned channel estimation solutions are difficult to be applied to the aeronautical THz UM-MIMO systems due to the unprecedentedly ultra-large array aperture, ultra-broad band, and ultra-high velocity. Compared with the sub-6 GHz or mmWave massive MIMO systems with limited aperture and bandwidth, the aeronautical THz UM-MIMO channels present the unique triple delay-beam-Doppler squint effects. To be specific, adopting the UPA form, the UMMIMO arrays mounted on the transceiver of aerial BSs or aircraft can be equipped with up to hundreds of antennas in the single horizontal or vertical dimension, resulting in the ultra-large array aperture even in a small physical size. If the direction of arrival is not perpendicular to the array, we can observe different propagation delays at different antennas for the same received signal filling this array aperture. Moreover, this delay gap can be as large as multiple symbol periods due to the usage of ultra-broadband $\mathrm{THz}$ communications. This indicates that the inter-symbol-interference can be nonnegligible even for the LoS link, and this phenomenon is termed as the delay squint effect of THz UM-MIMO (also named as spatial-frequency wideband effects in [28], [29] and aperture fill time effect in radar systems [30]), which is an inevitable challenge for THz UM-MIMO systems. Meanwhile, this delay squint effect can further introduce the beam squint effect, where the beam direction is a function of the operating frequency. This is primarily because radio waves at different frequencies would accumulate different phases given the same transmission distance, while the adjacent antenna spacing is designed according to the central carrier frequency. Hence, beam squint effect would pose undesired beam directions for the signals at marginal carrier frequencies. Furthermore, the high-speed mobility of aeronautical communications causes 




Fig. 2. A real-time flight tracking snapshot of civil aircrafts in south China, where the aircrafts generally fly along their fixed routes ${ }^{2}$.

large Doppler shift and the Doppler shift is also frequencydependent for aeronautical THz UM-MIMO with very large bandwidth. This phenomenon is called Doppler squint effect. Therefore, the aeronautical THz UM-MIMO systems present triple delay-beam-Doppler squint effects. However, recent researches mainly focus on the impact of beam squint effect on mmWave or $\mathrm{THz}$ systems [31]-[35]. To be specific, the impact of beam squint on compressive subspace estimation and the optimality of frequency-flat beamforming was studied in [31]. By projecting all frequencies to the central frequency and constructing the common analog Transmit Precoding (TPC) matrix for all subcarriers, several hybrid TPC schemes were proposed in [32] to design the analog and digital TPC matrices and mitigate the beam squint effect. The channel estimation schemes were proposed to exploit the characteristics of mmWave channels affected by beam squint for estimating the wideband mmWave massive MIMO channels [33]-[35], where the beam squint effect is not mitigated. To sum up, the triple squint effects are seldom considered in state-of-the-art channel estimation and hybrid beamforming solutions [21][29], [31]-[35] and can dramatically degrade the data transmission performance of $\mathrm{THz}$ UM-MIMO-based aeronautical communications. Consequently, an efficient signal processing paradigm for channel estimation and data transmission is invoked for enabling aeronautical THz UM-MIMO technique.

In this paper, we mainly investigate the THz UM-MIMObased aeronautical communication links connecting aircraft and aerial $\mathrm{BSs}$ in $\mathrm{SAGIN}^{3}$, where the practical triple squint effects of aeronautical THz UM-MIMO channel with LoS link will be considered. Specifically, for the airborne network in

\footnotetext{
${ }^{2}$ This real-time snapshot can be found on the website URL link: https://flightadsb.variflight.com/tracker/112.761836,29.084716/6.

${ }^{3}$ The proposed signal processing solution can also be applied to the space-space/space-air links between the UAVs and multiple aerial BSs, or between aircrafts/UAVs and multiple LEO satellites, etc, and the transmission links between the terrestrial stations built on high-altitude mountains and space-air networks. Furthermore, the research on space-ground or air-ground communications in SAGIN is beyond the scope of this paper, and it may be an important research direction of future work.
}

Fig. 1, the trajectories of aircrafts are usually regular along their fixed routes, as shown in Fig. 2. Based on this fact, the aerial BSs can be deployed near these trajectories to ensure that multiple aircrafts or UAVs can communicate with multiple aerial BSs for constituting the aeronautical ad hoc network. Since there are few other scatterers in the stratosphere except high altitude platforms for $\mathrm{THz}$ aeronautical communications, we mainly focus on the THz UM-MIMO channel with only LoS component between the aerial BS and the aircraft in this paper. More specifically, we consider that multiple aerial BSs can jointly serve a high-speed mobile aircraft through respective $\mathrm{THz}$ LoS links, and different aerial BSs can be cooperated via $\mathrm{THz}$ backbone links connecting different aerial BSs or the air-to-ground backbone links. To combat the multipath effect at the receiver of aircraft caused by multiple $\mathrm{THz}$ LoS links, the Orthogonal Frequency-Division Multiplexing (OFDM) technique will be applied to this aeronautical communication system ${ }^{4}$. Among the $\mathrm{THz}$ links aforementioned, the THz UM-MIMO-based aeronautical communication links connecting the aircrafts and aerial BSs are the most challenging to be established due to their fast time-varying fading characteristics. On the one hand, by exploiting the prior information (e.g., positioning, flight speed and direction, and posture information) at aerial BSs and aircrafts, some rough channel parameter estimates (e.g., angle and Doppler shift) can be acquired for facilitating the link establishment. On the other hand, these rough channel parameter estimates are not accurate enough for data transmission. Particularly, due to the exceedingly long link distance and extremely narrow beamwidth of aeronautical THz UM-MIMO, a slight deviation of angle parameter resulted from the positioning accuracy error and the posture rotation of antenna arrays mounted on transceiver would lead to the undesired beam pointing. Therefore, how to effectively leverage the prior information above to establish and track the fast time-varying links is vital for $\mathrm{THz}$ UM-MIMO-based aeronautical communications.

The proposed channel estimation and tracking solution can be divided into three stages, including the initial channel estimation for link establishment, data-aided channel tracking, and pilot-aided channel tracking. The frame structure is shown in Fig. 3, and the details are presented as follows:

- At the initial channel estimation stage, by utilizing the rough angle estimates acquired according to the positioning and flight posture information, the rough transmit beamforming and receive combining can be achieved to establish the $\mathrm{THz}$ UM-MIMO link, where the impact of delay-beam squint effects on both the transmitter and receiver can be significantly mitigated by employing a Grouping True-Time Delay Unit (GTTDU) module with low hardware cost.

- After the link establishment, the fine estimates of azimuth/elevation angles at both the transmitter and receiver,

${ }^{4}$ To meet the high quality-of-service requirement for hundreds of people in the aircraft simultaneously, the relatively complicated high-order modulation methods, i.e., OFDM and Quadrature Amplitude Modulation (QAM), can be utilized to enhance the data transmission rate and throughput in this paper. Moreover, due to the high Peak-to-Average Power Ratio (PAPR) in OFDM systems, Discrete Fourier Transform-Spread-OFDM (DFT-S-OFDM) technique is also the potential alternative for THz UM-MIMO-based aeronautical communication systems. 


\begin{tabular}{|c|c|c|c|c|}
\hline \multicolumn{2}{|c|}{$\begin{array}{c}\text { Aided with rough angle } \\
\text { estimates at BSs and aircraft }\end{array}$} & \multicolumn{2}{|c|}{$\begin{array}{c}\text { Fine Doppler } \\
\text { compensation }\end{array}$} \\
\hline $\begin{array}{c}\text { Fine angle } \\
\text { estimation at BSs }\end{array}$ & $\begin{array}{c}\text { Fine angle } \\
\text { estimation at aircraft }\end{array}$ & $\begin{array}{c}\text { Fine Doppler shift } \\
\text { estimation }\end{array}$ & $\begin{array}{c}\text { Path delay } \\
\text { estimation }\end{array}$ \\
\hline \begin{tabular}{|c|c|c|c|} 
Initial channel estimation \\
\hline $\begin{array}{c}\text { Data-aided } \\
\text { channel tracking }\end{array}$
\end{tabular} & $\begin{array}{c}\text { Data-aided } \\
\text { channel tracking }\end{array}$ & $\begin{array}{c}\text { Data-aided } \\
\text { channel tracking }\end{array}$ & $\cdots$ & $\cdots$ \\
\hline \begin{tabular}{|c|c|c|c|}
\hline $\begin{array}{c}\text { Angle tracking } \\
\text { at BSs }\end{array}$ & $\begin{array}{c}\text { Angle tracking } \\
\text { at aircraft }\end{array}$ & $\begin{array}{c}\text { Doppler shift } \\
\text { estimation }\end{array}$ & $\begin{array}{c}\text { Path delay } \\
\text { estimation }\end{array}$ \\
\hline
\end{tabular}
\end{tabular}

Fig. 3. Frame structure of the proposed channel estimation and tracking solution.

Doppler shifts, and path delays at the receiver are then obtained, where the rough Doppler shift estimates are utilized to compensate the received signals for improved parameter estimation. For the fine azimuth/elevation angle estimation, the UM hybrid array can be equivalently considered as a low-dimensional fully-digital array by employing a reconfigurable Radio Frequency (RF) selection network with dedicated connection pattern. In this way, the accurate estimates of azimuth/elevation angles at BSs and aircraft can be separately acquired using the proposed prior-aided iterative angle estimation algorithm. These fine angle estimates can be used not only to achieve the more precise beam alignment, but also to refine the GTTDU module at the transceiver for further eliminating the delay-beam squint effects. Meanwhile, thanks to the large beam alignment gain and the sufficient receive Signal-toNoise Ratio (SNR), the Doppler shifts can be accurately estimated based on the proposed prior-aided iterative Doppler shift estimation algorithm, where the Doppler squint effect can be attenuated vastly by compensating the received signals with the rough Doppler shift estimates. On this basis, path delays and channel gains can be estimated subsequently, where Doppler squint effect can be also attenuated vastly via fine compensation process.

- At the data transmission stage, a Data-Aided DecisionDirected (DADD)-based channel tracking algorithm is developed to track the beam-aligned effective channels, where the correctly decoded data will be regarded as the known signals to estimate channel coefficients.

- The pilot-aided channel tracking is proposed when the data-aided channel tracking is ineffective. At this stage, an equivalent fully-digital sparse array will be formed by reconfiguring the connection pattern of the RF selection network, where the angle ambiguity issue derived from sparse array can be addressed with the aid of the previously estimated angles at the receiver. Once the precise beam alignment is achieved again, the Doppler shift and path delay estimation can be executed similar to the initial channel estimation stage, and then the transceiver will enter the data transmission stage again.

The main contributions of our proposed scheme are summarized as the following aspects:

- $\mathrm{THz}$ UM-MIMO-based aeronautical communication channels exhibit the huge spatial dimension and very fast time-variability. To reduce the training overhead, we propose a parametric channel estimation and tracking solution. At the stages of initial channel estimation and pilot-aided channel tracking, by exploiting the proposed prior-aided iterative angle and Doppler shift estimation algorithms, the proposed solution can acquire the fine estimates of channel angles, Doppler shifts, and path delays, whereby some rough channel parameter estimates are leveraged to improve the estimated accuracy and reduce the pilot overhead. At the data transmission stage, to further save the pilot overhead, the proposed DADDbased channel tracking algorithm can reliably track the fast time-varying channel gains of the effective beamaligned link.

- The proposed scheme can effectively overcome the unique triple delay-beam-Doppler squint effects of aeronautical THz UM-MIMO communications. Note that this triple squint effects are rarely observed and investigated in the sub-6 $\mathrm{GHz}$ or mmWave massive MIMO systems due to the limited aperture and bandwidth. To cope with the delay-beam squint effects, we propose the low-cost GTTDU module at the transceiver, which can compensate the signal transmission delays at different antenna group with the aid of navigation information. In this way, the delay-beam squint effects can be significantly mitigated and the sufficient receive SNR can be guaranteed to establish the THz link. Also, the designed Rotman lensbased GTTDU module in Section VIII provides a feasible implementation architecture of the tunable TTD module based Phase Shift Network (PSN), which would be a potential direction for the future research work. Furthermore, by utilizing the proposed prior-aided iterative angle and Doppler shift estimation algorithms to further mitigate the impact of beam and Doppler squint effects, the fine angle and Doppler shift estimates can be acquired for the following data transmission.

- We introduce a reconfigurable RF selection network to obtain the equivalent low-dimensional fully-digital array by designing the dedicated connection pattern. On this basis, the robust array signal processing techniques such as Two-Dimensional Unitary ESPRIT (TDU-ESPRIT) [36], [37] can be utilized to accurately estimate and track the azimuth/elevation angles at the transceiver. Particu- 
larly, by reconfiguring the connection pattern of the RF selection network, the equivalent fully-digital sparse array can be obtained for improved angle estimation accuracy at the pilot-aided channel tracking stage, where angle ambiguity issue can be addressed well based on the previously estimated angles.

- The Cramér-Rao Lower Bounds (CRLBs) of dominant channel parameters are derived based on the effective received signal models. Particularly, at the pilot-aided channel tracking stage, the CRLBs of angles are derived to theoretically verify the improved estimation accuracy by employing the sparse array. Simulations results have the good tightness with the analytical CRLBs, which testifies the good performance of the proposed scheme.

The remainder of this paper is organized as follows. Section II introduces the system model, including the signal transmission and channel models with triple squint effects. The initial channel parameter estimation stage, including the estimations of azimuth/elevation angles at BSs and aircraft, Doppler shifts, path delays, and channel gains, is illustrated in Section III. The DADD-based channel tracking and the pilotaided channel tracking methods are proposed in Sections IV and $\mathrm{V}$, respectively. Section VI presents the performance analysis on CRLB and computational complexity. The numerical evaluations is given in Section VII. Finally, Section VIII concludes this paper.

Throughout this paper, boldface lower and upper-case symbols denote column vectors and matrices, respectively. $(\cdot)^{*}$, $(\cdot)^{\mathrm{T}},(\cdot)^{\mathrm{H}},(\cdot)^{-1}$, and $|\cdot|$ denote the conjugate, transpose, Hermitian transpose, matrix inversion, and modulus operators, respectively. $\|\boldsymbol{a}\|_{2}$ and $\|\boldsymbol{A}\|_{F}$ are the $\ell_{2}$-norm of $\boldsymbol{a}$ and the Frobenius norm of $\boldsymbol{A}$, respectively. The Kronecker and Hadamard product operations are denoted by $\otimes$ and $\circ$, respectively. $\langle\boldsymbol{a}, \boldsymbol{b}\rangle$ expresses the inner product of vectors $\boldsymbol{a}$ and $\boldsymbol{b} . \mathbf{0}_{n}$ and $\boldsymbol{I}_{n}$ denote the vector of size $n$ with all the elements being 0 and the $n \times n$ identity matrix, respectively. $|\mathcal{Q}|_{c}$ is the cardinality of the set $\mathcal{Q}$, and $\{\mathcal{Q}\}_{n}$ denotes the $n$th element of the ordered set $\mathcal{Q} .[a]_{\mathcal{Q}}$ denotes the subvector containing the elements of $\boldsymbol{a}$ indexed in the ordered set $\mathcal{Q}$. $[\boldsymbol{a}]_{m}$ and $[\boldsymbol{A}]_{m, n}$ denotes the $m$ th element of $\boldsymbol{a}$ and the $m$ th-row and the $n$ th-column element of $\boldsymbol{A}$, respectively. $\operatorname{diag}(\boldsymbol{a})$ is the diagonal matrix with the elements of $\boldsymbol{a}$ at its diagonal entries. $\partial(\cdot)$ and $\partial^{2}(\cdot)$ are the first- and second-order partial derivative operations, respectively. Finally, $\mathbb{E}(\cdot)$ and $\Re\{\cdot\}$ denote the expectation and real part of the argument, respectively.

\section{SySTEM MODEL}

In this section, we will formulate the signal transmission and channel models with LoS link for THz UM-MIMO-based aeronautical communications, where the full-dimensional UMMIMO channel model using UPAs involves azimuth and elevation angles [37], [38]. Fig. 4(a) depicts the specific scenario that $L$ aerial BSs jointly serve an aircraft through respective $\mathrm{THz}$ LoS links. The aerial BSs and aircraft adopt the hybrid beamforming structure with a sub-connected PSN [4], [9], where the sub-connected PSNs at BSs can be simplified as analog beamforming to serve the assigned aircraft. The specific configurations of these antenna arrays are as follows. The total number of antennas at BS arrays is $N_{\mathrm{BS}}=N_{\mathrm{BS}}^{\mathrm{h}} N_{\mathrm{BS}}^{\mathrm{v}}$, where $N_{\mathrm{BS}}^{\mathrm{h}}$ and $N_{\mathrm{BS}}^{\mathrm{v}}$ are the numbers of antennas in horizontal and vertical directions, respectively. Due to the sub-connected PSN adopted at aircraft, we define $\widetilde{I}_{\mathrm{AC}}^{\mathrm{h}}\left(M_{\mathrm{AC}}^{\mathrm{h}}\right)$ and $\widetilde{I}_{\mathrm{AC}}^{\mathrm{v}}$ $\left(M_{\mathrm{AC}}^{\mathrm{v}}\right)$ as the numbers of subarrays (antennas within each subarray) in horizontal and vertical directions, respectively; while $N_{\mathrm{AC}}^{\mathrm{h}}=\widetilde{I}_{\mathrm{AC}}^{\mathrm{h}} M_{\mathrm{AC}}^{\mathrm{h}}$ and $N_{\mathrm{AC}}^{\mathrm{v}}=\widetilde{I}_{\mathrm{AC}}^{\mathrm{v}} M_{\mathrm{AC}}^{\mathrm{v}}$ are the numbers of antennas in horizontal and vertical directions of array, respectively. Then, the total numbers of antennas in each subarray and the whole antenna array are $M_{\mathrm{AC}}=M_{\mathrm{AC}}^{\mathrm{h}} M_{\mathrm{AC}}^{\mathrm{v}}$ and $N_{\mathrm{AC}}=N_{\mathrm{AC}}^{\mathrm{h}} N_{\mathrm{AC}}^{\mathrm{v}}$, respectively. Clearly, the aircraft and BS are equipped with $L=\widetilde{I}_{\mathrm{AC}}^{\mathrm{h}} \widetilde{I}_{\mathrm{AC}}^{\mathrm{v}} \mathrm{RF}$ chains and only one RF chain, respectively, and each subarray and the corresponding $\mathrm{RF}$ chain mounted on aircraft are assigned to one BS.

According to the frame structure in Fig. 3, the azimuth/elevation angles at BSs and aircraft are estimated in the Uplink (UL) and Downlink (DL), respectively, and OFDM with $K$ subcarriers is adopted. The UL baseband signal $y_{\mathrm{UL}, l}^{[m]}[k]$ received by the $l$ th $\mathrm{BS}$ at the $k$ th subcarrier of the $m$ th OFDM symbol can be expressed as

$$
\begin{aligned}
y_{\mathrm{UL}, l}^{[m]}[k]= & \sqrt{P_{l}} \boldsymbol{q}_{\mathrm{RF}, l}^{\mathrm{H}} \boldsymbol{H}_{\mathrm{UL}, l}^{[m]}[k] \boldsymbol{P}_{\mathrm{RF}} \boldsymbol{P}_{\mathrm{BB}}^{[m]}[k] \boldsymbol{s}_{\mathrm{UL}}^{[m]}[k] \\
& +\boldsymbol{q}_{\mathrm{RF}, l}^{\mathrm{H}} \boldsymbol{n}_{\mathrm{UL}, l}^{[m]}[k],
\end{aligned}
$$

where $1 \leq l \leq L, 1 \leq k \leq K$, and $P_{l}$ is the transmit power. In (1), $\boldsymbol{q}_{\mathrm{RF}, l} \in \mathbb{C}^{N_{\mathrm{BS}}}$ is the analog combining vector of the $l$ th $\mathrm{BS}, \boldsymbol{P}_{\mathrm{RF}} \in \mathbb{C}^{N_{\mathrm{AC}} \times L}$ and $\boldsymbol{P}_{\mathrm{BB}}^{[m]}[k] \in \mathbb{C}^{L \times L}$ are the analog and digital precoding matrices at aircraft, respectively, while $\boldsymbol{H}_{\mathrm{UL}, l}^{[m]}[k] \in \mathbb{C}^{N_{\mathrm{BS}} \times N_{\mathrm{AC}}}$ is the UL effective baseband channel matrix, $s_{\mathrm{UL}}^{[m]}[k] \in \mathbb{C}^{L}$ is the transmitted signal vector, and $\boldsymbol{n}_{\mathrm{UL}, l}^{[m]}[k] \in \mathbb{C}^{N_{\mathrm{BS}}}$ is the complex Additive White Gaussian Noise (AWGN) vector with the covariance $\sigma_{n}^{2}$, i.e., $\boldsymbol{n}_{\mathrm{UL}, l}^{[m]}[k] \sim$ $\mathcal{C N}\left(\mathbf{0}_{N_{\mathrm{BS}}}, \sigma_{n}^{2} \boldsymbol{I}_{N_{\mathrm{BS}}}\right)$. Similarly, the DL baseband signal vector $\boldsymbol{y}_{\mathrm{DL}}^{[n]}[k] \in \mathbb{C}^{L}$ received by aircraft at the $k$ th subcarrier of the $n$th OFDM symbol is given by

$$
\begin{aligned}
\boldsymbol{y}_{\mathrm{DL}}^{[n]}[k]= & \left(\boldsymbol{W}_{\mathrm{BB}}^{[n]}[k]\right)^{\mathrm{H}} \boldsymbol{W}_{\mathrm{RF}}^{\mathrm{H}}\left(\sum_{l=1}^{L} \sqrt{P_{l}} \boldsymbol{H}_{\mathrm{DL}, l}^{[n]}[k] \boldsymbol{f}_{\mathrm{RF}, l} s_{\mathrm{DL}, l}^{[n]}[k]\right. \\
& \left.+\boldsymbol{n}_{\mathrm{DL}}^{[n]}[k]\right),
\end{aligned}
$$

where $\boldsymbol{W}_{\mathrm{RF}} \in \mathbb{C}^{N_{\mathrm{AC}} \times L}$ and $\boldsymbol{W}_{\mathrm{BB}}^{[n]}[k] \in \mathbb{C}^{L \times L}$ are the ana$\log$ and digital combining matrices at aircraft, respectively, $\boldsymbol{f}_{\mathrm{RF}, l} \in \mathbb{C}^{N_{\mathrm{BS}}}$ is the analog precoding vector of the $l$ th $\mathrm{BS}$, while $\boldsymbol{H}_{\mathrm{DL}, l}^{[n]}[k] \in \mathbb{C}^{N_{\mathrm{AC}} \times N_{\mathrm{BS}}}$ is the DL effective baseband channel matrix, and $s_{\mathrm{DL}, l}^{[n]}[k]$ and $\boldsymbol{n}_{\mathrm{DL}, l}^{[n]}[k] \in \mathbb{C}^{N_{\mathrm{AC}}}$ are the transmitted pilot signal (or the modulated/coded data) and the AWGN vector (similar to $\boldsymbol{n}_{\mathrm{UL}, l}^{[m]}[k]$ ), respectively.

To illustrate the delay squint effect of THz UM-MIMO channels, we take the antenna array at BS as an example as shown in Fig. 4(b). Specifically, the first $(1,1)$ th antenna element can be regarded as the reference point, and define $\boldsymbol{r}=\left(\sin \left(\theta_{l}^{\mathrm{BS}}\right) \cos \left(\varphi_{l}^{\mathrm{BS}}\right), \sin \left(\varphi_{l}^{\mathrm{BS}}\right), \cos \left(\theta_{l}^{\mathrm{BS}}\right) \cos \left(\varphi_{l}^{\mathrm{BS}}\right)\right)$ as the unit direction vector, where $\theta_{l}^{\mathrm{BS}}$ and $\varphi_{l}^{\mathrm{BS}}$ are the azimuth and elevation angles associated with the $l$ th BS, respectively. 


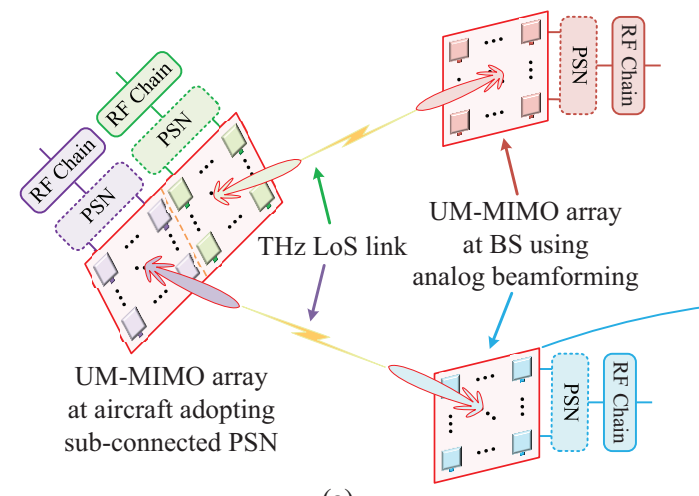

(a)

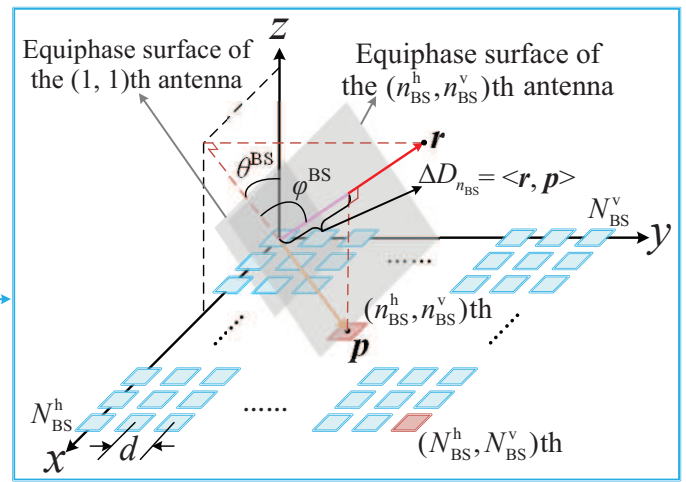

(b)

Fig. 4. The structure diagram of the antenna arrays at transceiver: (a) $L=2$ BSs that use analog beamforming communicate with aircraft adopting subconnected PSN through respective LoS links, and (b) takes the UPA at BS with size of $N_{\mathrm{BS}}^{\mathrm{h}} \times N_{\mathrm{BS}}^{\mathrm{v}}$ as an example to illustrate the delay squint effect of THz UM-MIMO array.

Defining the $\left(n_{\mathrm{BS}}^{\mathrm{h}}, n_{\mathrm{BS}}^{\mathrm{v}}\right)$ th antenna as the $n_{\mathrm{BS}}$ th antenna with $n_{\mathrm{BS}}=\left(n_{\mathrm{BS}}^{\mathrm{v}}-1\right) N_{\mathrm{BS}}^{\mathrm{h}}+n_{\mathrm{BS}}^{\mathrm{h}}$, its direction vector relative to the reference antenna is $\boldsymbol{p}=\left(\left(n_{\mathrm{BS}}^{\mathrm{h}}-1\right) d,\left(n_{\mathrm{BS}}^{\mathrm{v}}-1\right) d, 0\right)$, where $d$ denotes the adjacent antenna spacing with half-wavelength. The wave path-difference between the $n_{\mathrm{BS}}$ th antenna and the first antenna, denoted by $\Delta D_{n_{\mathrm{BS}}}$, is equal to the distance between the equiphase surfaces of these two antennas, i.e., $\Delta D_{n_{\mathrm{BS}}}=\langle\boldsymbol{r}, \boldsymbol{p}\rangle=\left(n_{\mathrm{BS}}^{\mathrm{h}}-1\right) d \sin \left(\theta_{l}^{\mathrm{BS}}\right) \cos \left(\varphi_{l}^{\mathrm{BS}}\right)+\left(n_{\mathrm{BS}}^{\mathrm{v}}-\right.$ 1) $d \sin \left(\varphi_{l}^{\mathrm{BS}}\right)$. Denoting $\tau_{l}^{\left[n_{\mathrm{BS}}\right]}$ as the transmission delay from the $n_{\mathrm{BS}}$ th antenna to the first antenna for the $l$ th BS, we can obtain $\tau_{l}^{\left[n_{\mathrm{BS}}\right]}=\Delta D_{n_{\mathrm{BS}}} / c$ with $c$ being the speed of light. Note that $\tau_{l}^{\left[n_{\mathrm{BS}}\right]}$ is related to the antenna index and the azimuth/elevation angles. When the signal direction is not perpendicular to the array and $n_{\mathrm{BS}}$ is large, $\tau_{l}^{\left[n_{\mathrm{BS}}\right]}$ can be even larger than the symbol period $T_{s}{ }^{5}$, which compels higher demands on the signal processing at the receiver, especially for the analog or hybrid beamforming architecture. Therefore, the delay squint effect needs to be taken into account for aeronautical THz UM-MIMO systems.

Considering the channel reciprocity in time division duplex systems, we focus on the formulation of DL channel matrix next. According to the channel model in [34], [39], define the DL passband channel matrix in the spatial-delay domain as $\overline{\boldsymbol{H}}_{\mathrm{DL}, l}^{(t)}(\tau) \in \mathbb{C}^{N_{\mathrm{AC}} \times N_{\mathrm{BS}}}$ at time $t$ corresponding to the $l$ th BS, whose the $\left(n_{\mathrm{AC}}, n_{\mathrm{BS}}\right)$ th element, i.e., $\left[\overline{\boldsymbol{H}}_{\mathrm{DL}, l}^{(t)}(\tau)\right]_{n_{\mathrm{AC}}, n_{\mathrm{BS}}}$, can be expressed as

$$
\begin{aligned}
& {\left[\overline{\boldsymbol{H}}_{\mathrm{DL}, l}^{(t)}(\tau)\right]_{n_{\mathrm{AC}}, n_{\mathrm{BS}}}} \\
& =\sqrt{G_{l}} \alpha_{l} e^{\mathrm{j} 2 \pi \psi_{l} t} \delta(\tau-\tau_{l}-\underbrace{\left(\tau_{l}^{\left[n_{\mathrm{AC}}\right]}+\tau_{l}^{\left[n_{\mathrm{BS}}\right]}\right)}_{\text {Delay squint }}),
\end{aligned}
$$

where $1 \leq n_{\mathrm{AC}} \leq N_{\mathrm{AC}}, 1 \leq n_{\mathrm{BS}} \leq N_{\mathrm{BS}}, G_{l}$ and $\alpha_{l} \sim$ $\mathcal{C N}\left(0, \sigma_{\alpha}^{2}\right)$ are the large-scale fading gain of communication

${ }^{5} \mathrm{We}$ consider an extreme scenario that the impinging signal comes from the diagonal direction of UPA of size $\left(n_{\mathrm{BS}}+1\right) \times\left(n_{\mathrm{BS}}+1\right)$, and those $\left(n_{\mathrm{BS}}+1\right)$ diagonal antennas consist of the Uniform Linear Array (ULA) of size $\left(n_{\mathrm{BS}}+1\right)$ with $\sqrt{2} d$ antenna spacing. When angle $\theta_{l}^{\mathrm{BS}}=60^{\circ}$, carrier frequency $f_{c}=0.1 \mathrm{THz}$, and bandwidth $f_{s}=1 \mathrm{GHz}$ for the typical $\mathrm{THz}$ UM-MIMO aeronautical communication scenario, $n_{\mathrm{BS}}=200$ antennas will make its filling time satisfy $\tau_{l}^{\left[n_{\mathrm{BS}}\right]}=\frac{\sqrt{2} n_{\mathrm{BS}} \sin \left(\theta_{l}^{\mathrm{BS}}\right)}{2 f_{c}} \approx 1.225 T_{s}$. link and the channel gain ${ }^{6}$, respectively, $\psi_{l}=\underline{v}_{l} / \lambda_{c}$ denotes the Doppler shift with $\underline{v}_{l}$ and $\lambda_{c}$ being the relative radial velocity and carrier wavelength, respectively, $f_{c}$ is the corresponding carrier frequency, $\tau_{l}^{\left[n_{\mathrm{AC}}\right]}$ denotes the transmission delay between the $n_{\mathrm{AC}}$ th antenna $\left(n_{\mathrm{AC}}=\left(n_{\mathrm{AC}}^{\mathrm{v}}-1\right) N_{\mathrm{AC}}^{\mathrm{h}}+n_{\mathrm{AC}}^{\mathrm{h}}\right.$, and it also the $\left(n_{\mathrm{AC}}^{\mathrm{h}}, n_{\mathrm{AC}}^{\mathrm{v}}\right)$ th antenna of UPA at aircraft) and its reference point, and $\delta(\cdot)$ and $\tau_{l}$ are the Dirac impulse function and the path delay, respectively. After some algebraic transformations, the DL spatial-frequency channel matrix $\boldsymbol{H}_{\mathrm{DL}, l}^{[n]}[k]$ in (2) at the $k$ th subcarrier of the $n$th OFDM symbol can be expressed as

$$
\begin{aligned}
\boldsymbol{H}_{\mathrm{DL}, l}^{[n]}[k]= & \sqrt{G_{l}} \alpha_{l} e^{\mathrm{j} 2 \pi \psi_{l, k}(n-1) T_{\mathrm{sym}}} e^{-\mathrm{j} 2 \pi\left(\frac{k-1}{K}-\frac{1}{2}\right) f_{s} \tau_{l}} \\
& \times \boldsymbol{A}_{\mathrm{DL}, l}[k],
\end{aligned}
$$

where $T_{\text {sym }}$ and $f_{s}$ denote the duration time of an OFDM symbol and system bandwidth, respectively, $\psi_{l, k}=\psi_{z, l}+$ $\frac{v_{l}}{c}\left(\frac{k-1}{K}-\frac{1}{2}\right) f_{s}$ is the frequency-dependent Doppler shift at the $k$ th subcarrier with $\psi_{z, l}$ being the Doppler shift of the central carrier frequency $f_{z}$ (wavelength $\lambda_{z}$ ) and $\frac{\underline{v}_{l}}{c}\left(\frac{k-1}{K}-\frac{1}{2}\right) f_{s}$ being the Doppler squint part due to the large bandwidth in $\mathrm{THz}$ communications, and $\boldsymbol{A}_{\mathrm{DL}, l}[k] \in \mathbb{C}^{N_{\mathrm{AC}} \times N_{\mathrm{BS}}}$ is the DL array response matrix associated with the array response vectors at aircraft and the $l$ th BS, given by

$$
\begin{aligned}
\boldsymbol{A}_{\mathrm{DL}, l}[k]= & \underbrace{\left(\boldsymbol{a}_{\mathrm{AC}}\left(\mu_{l}^{\mathrm{AC}}, \nu_{l}^{\mathrm{AC}}\right) \boldsymbol{a}_{\mathrm{BS}}^{\mathrm{H}}\left(\mu_{l}^{\mathrm{BS}}, \nu_{l}^{\mathrm{BS}}\right)\right)}_{\boldsymbol{A}_{\mathrm{DL}, l}} \\
& \circ \underbrace{\left(\overline{\boldsymbol{a}}_{\mathrm{AC}}\left(\mu_{l}^{\mathrm{AC}}, \nu_{l}^{\mathrm{AC}}, k\right) \overline{\boldsymbol{a}}_{\mathrm{BS}}^{\mathrm{H}}\left(\mu_{l}^{\mathrm{BS}}, \nu_{l}^{\mathrm{BS}}, k\right)\right)}_{\overline{\boldsymbol{A}}_{\mathrm{DL}, l}[k](\text { Beam squint component })},
\end{aligned}
$$

where $\mu_{l}^{\mathrm{AC}}=\pi \sin \left(\theta_{l}^{\mathrm{AC}}\right) \cos \left(\varphi_{l}^{\mathrm{AC}}\right) \quad\left(\mu_{l}^{\mathrm{BS}}=\right.$ $\left.\pi \sin \left(\theta_{l}^{\mathrm{BS}}\right) \cos \left(\varphi_{l}^{\mathrm{BS}}\right)\right)$ and $\nu_{l}^{\mathrm{AC}}=\pi \sin \left(\varphi_{l}^{\mathrm{AC}}\right)$ $\left(\nu_{l}^{\mathrm{BS}}=\pi \sin \left(\varphi_{l}^{\mathrm{BS}}\right)\right)$ are the horizontally and vertically virtual angles at aircraft (the $l$ th $\mathrm{BS}$ ), respectively, $\boldsymbol{A}_{\mathrm{DL}, l}$ is the conventional DL array response matrix without beam squint

${ }^{6}$ Due to the negligible frequency-dependent attenuation of $\mathrm{THz}$ communication links (e.g., atmospheric molecular absorption) in the stratosphere and above [13], [14], the channel gain $\alpha_{l}$ can be modeled as a frequency flat coefficient, which is different from the frequency-dependent channel coefficient in [39]. 
effect at aircraft and BS, and $\overline{\boldsymbol{A}}_{\mathrm{DL}, l}[k]$ is the corresponding array response squint matrix considering beam squint effect. In (5), $\boldsymbol{a}_{\mathrm{AC}}\left(\mu_{l}^{\mathrm{AC}}, \nu_{l}^{\mathrm{AC}}\right)=\boldsymbol{a}_{\mathrm{v}}\left(\nu_{l}^{\mathrm{AC}}, N_{\mathrm{AC}}^{\mathrm{v}}\right) \otimes \boldsymbol{a}_{\mathrm{h}}\left(\mu_{l}^{\mathrm{AC}}, N_{\mathrm{AC}}^{\mathrm{h}}\right)$ and $\boldsymbol{a}_{\mathrm{BS}}\left(\mu_{l}^{\mathrm{BS}}, \nu_{l}^{\mathrm{BS}}\right)=\boldsymbol{a}_{\mathrm{v}}\left(\nu_{l}^{\mathrm{BS}}, N_{\mathrm{BS}}^{\mathrm{v}}\right) \otimes \boldsymbol{a}_{\mathrm{h}}\left(\mu_{l}^{\mathrm{BS}}, N_{\mathrm{BS}}^{\mathrm{h}}\right)$ are the general array response vectors at aircraft and the $l$ th BS [37], respectively, and $\overline{\boldsymbol{a}}_{\mathrm{AC}}\left(\mu_{l}^{\mathrm{AC}}, \nu_{l}^{\mathrm{AC}}, k\right)=\overline{\boldsymbol{a}}_{\mathrm{v}}\left(\nu_{l}^{\mathrm{AC}}, N_{\mathrm{AC}}^{\mathrm{v}}, k\right) \otimes \overline{\boldsymbol{a}}_{\mathrm{h}}\left(\mu_{l}^{\mathrm{AC}}, N_{\mathrm{AC}}^{\mathrm{h}}, k\right)$ and $\overline{\boldsymbol{a}}_{\mathrm{BS}}\left(\mu_{l}^{\mathrm{BS}}, \nu_{l}^{\mathrm{BS}}, k\right)=\overline{\boldsymbol{a}}_{\mathrm{v}}\left(\nu_{l}^{\mathrm{BS}}, N_{\mathrm{BS}}^{\mathrm{v}}, k\right) \otimes \overline{\boldsymbol{a}}_{\mathrm{h}}\left(\mu_{l}^{\mathrm{BS}}, N_{\mathrm{BS}}^{\mathrm{h}}, k\right)$ are the frequency-dependent array response squint vectors, respectively. Moreover, the vectors at aircraft, i.e., the horizontal/vertical steering vectors $\boldsymbol{a}_{\mathrm{h}}\left(\mu_{l}^{\mathrm{AC}}, N_{\mathrm{AC}}^{\mathrm{h}}\right)$ and $\boldsymbol{a}_{\mathrm{v}}\left(\nu_{l}^{\mathrm{AC}}, N_{\mathrm{AC}}^{\mathrm{v}}\right)$, and the horizontal/vertical steering squint vectors $\overline{\boldsymbol{a}}_{\mathrm{h}}\left(\mu_{l}^{\mathrm{AC}}, N_{\mathrm{AC}}^{\mathrm{h}}, k\right)$ and $\overline{\boldsymbol{a}}_{\mathrm{v}}\left(\nu_{l}^{\mathrm{AC}}, N_{\mathrm{AC}}^{\mathrm{v}}, k\right)$ can be further written as

$$
\begin{aligned}
& \boldsymbol{a}_{\mathrm{h}}\left(\mu_{l}^{\mathrm{AC}}, N_{\mathrm{AC}}^{\mathrm{h}}\right)=\left[1 e^{\mathrm{j} \mu_{l}^{\mathrm{AC}}} \cdots e^{\mathrm{j}\left(N_{\mathrm{AC}}^{\mathrm{h}}-1\right) \mu_{l}^{\mathrm{AC}}}\right]^{\mathrm{T}}, \\
& \boldsymbol{a}_{\mathrm{v}}\left(\nu_{l}^{\mathrm{AC}}, N_{\mathrm{AC}}^{\mathrm{v}}\right)=\left[\begin{array}{lll}
1 e^{\mathrm{j} \nu_{l}^{\mathrm{AC}}} \cdots & e^{\mathrm{j}\left(N_{\mathrm{AC}}^{\mathrm{v}}-1\right) \nu_{l}^{\mathrm{AC}}}
\end{array}\right]^{\mathrm{T}}, \\
& \overline{\boldsymbol{a}}_{\mathrm{h}}\left(\mu_{l}^{\mathrm{AC}}, N_{\mathrm{AC}}^{\mathrm{h}}, k\right) \\
& =\left[1 e^{\mathrm{j}\left(\frac{k-1}{K}-\frac{1}{2}\right) \frac{f_{s}}{f_{z}} \mu_{l}^{\mathrm{AC}}} \cdots e^{\mathrm{j}\left(\frac{k-1}{K}-\frac{1}{2}\right) \frac{f_{s}}{f_{z}}\left(N_{\mathrm{AC}}^{\mathrm{h}}-1\right) \mu_{l}^{\mathrm{AC}}}\right]^{\mathrm{T}}, \\
& \overline{\boldsymbol{a}}_{\mathrm{v}}\left(\nu_{l}^{\mathrm{AC}}, N_{\mathrm{AC}}^{\mathrm{v}}, k\right) \\
& =\left[1 e^{\mathrm{j}\left(\frac{k-1}{K}-\frac{1}{2}\right) \frac{f_{s}}{f_{z}} \nu_{l}^{\mathrm{AC}}} \cdots e^{\mathrm{j}\left(\frac{k-1}{K}-\frac{1}{2}\right) \frac{f_{s}}{f_{z}}\left(N_{\mathrm{AC}}^{\mathrm{V}}-1\right) \nu_{l}^{\mathrm{AC}}}\right]^{\mathrm{T}} .
\end{aligned}
$$

Note that the vectors at BSs, i.e., $\boldsymbol{a}_{\mathrm{h}}\left(\mu_{l}^{\mathrm{BS}}, N_{\mathrm{BS}}^{\mathrm{h}}\right)$, $\boldsymbol{a}_{\mathrm{v}}\left(\nu_{l}^{\mathrm{BS}}, N_{\mathrm{BS}}^{\mathrm{v}}\right), \quad \overline{\boldsymbol{a}}_{\mathrm{h}}\left(\mu_{l}^{\mathrm{BS}}, N_{\mathrm{BS}}^{\mathrm{h}}, k\right), \quad$ and $\quad \overline{\boldsymbol{a}}_{\mathrm{v}}\left(\nu_{l}^{\mathrm{BS}}, N_{\mathrm{BS}}^{\mathrm{v}}, k\right)$, have the similar definitions and expressions to (6)-(9), and their details are omitted for simplicity. The detailed derivation of DL channel matrix $\boldsymbol{H}_{\mathrm{DL}, l}^{[n]}[k]$ can be found in Appendix A.

Similar to (4), the UL spatial-frequency baseband channel matrix $\boldsymbol{H}_{\mathrm{UL}, l}^{[m]}[k]$ in (1) at the $k$ th subcarrier of the $m$ th OFDM symbol corresponding to the $l$ th BS can be formulated as

$$
\boldsymbol{H}_{\mathrm{UL}, l}^{[m]}[k]=\sqrt{G_{l}} \alpha_{l} e^{\mathrm{j} 2 \pi \psi_{l, k}(m-1) T_{\mathrm{sym}}} \boldsymbol{A}_{\mathrm{UL}, l}[k],
$$

where the UL array response matrix $\boldsymbol{A}_{\mathrm{UL}, l}[k] \in \mathbb{C}^{N_{\mathrm{BS}} \times N_{\mathrm{AC}}}$ is

$$
\begin{aligned}
\boldsymbol{A}_{\mathrm{UL}, l}[k]= & \underbrace{\left(\boldsymbol{a}_{\mathrm{BS}}\left(\mu_{l}^{\mathrm{BS}}, \nu_{l}^{\mathrm{BS}}\right) \boldsymbol{a}_{\mathrm{AC}}^{\mathrm{H}}\left(\mu_{l}^{\mathrm{AC}}, \nu_{l}^{\mathrm{AC}}\right)\right)}_{\boldsymbol{A}_{\mathrm{UL}, l}} \\
& \circ \underbrace{\left(\overline{\boldsymbol{a}}_{\mathrm{BS}}\left(\mu_{l}^{\mathrm{BS}}, \nu_{l}^{\mathrm{BS}}, k\right) \overline{\boldsymbol{a}}_{\mathrm{AC}}^{\mathrm{H}}\left(\mu_{l}^{\mathrm{AC}}, \nu_{l}^{\mathrm{AC}}, k\right)\right)}_{\overline{\boldsymbol{A}}_{\mathrm{UL}, l}[k](\text { Beam squint component })} .
\end{aligned}
$$

\section{INITIAL ChANNEL Estimation}

As shown in Fig. 3, at the initial channel estimation stage, the fine azimuth/elevation angles at BSs and aircraft, Doppler shifts, and path delays are estimated successively. At this stage, according to the positioning and flight posture information acquired in aeronautical systems, some rough channel parameter estimates (e.g., angle and Doppler shift) can be utilized to establish the initial THz UM-MIMO link. Due to the positioning accuracy error and the posture rotations of antenna arrays mounted on aerial BSs and aircraft, these rough channel parameter estimates are not accurate enough for data

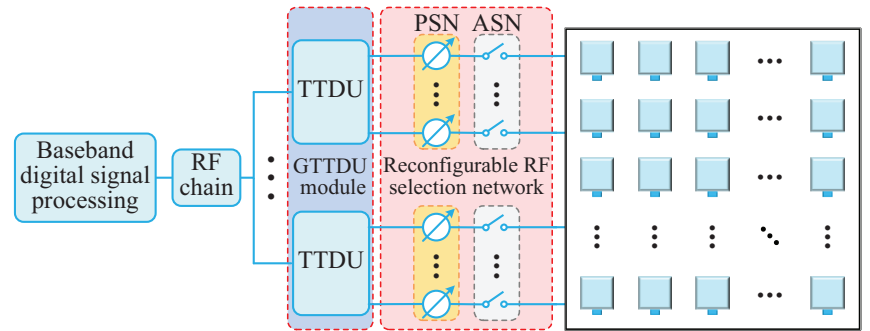

Fig. 5. The transceiver structure corresponding to one RF, where this RF chain connects with the antenna array via the GTTDU module and the reconfigurable RF selection network consisting of a sub-connected PSN and an ASN.

transmission. Therefore, the accurate acquisition of dominant channel parameters is still indispensable.

To overcome the delay-beam squint effects of THz UMMIMO array, the fully-digital array architecture with each antenna equipping a dedicated RF chain is preferred, but the involved prohibitive hardware cost and power consumption make it impracticable. Moreover, the aforementioned hybrid beamforming and channel estimation schemes [31]-[35] utilize some signal processing methods to attenuate the impact of delay-beam squint effects on the results, rather than eliminate these effects during signal transmission. Therefore, those processing methods are only suitable for the terrestrial mmWave or $\mathrm{THz}$ cellular networks with abundant scatterers, where the receiver in short-distance transmission (at most hundreds of meters) can receive the signals affected by delay-beam squint effects. However, for THz UM-MIMO-based aeronautical communication systems that rely on the long-distance transmission of LoS link (up to hundreds of kilometers) without supernumerary scatterers, the receiver will most likely fail to receive the signals at marginal carrier frequencies due to the very narrow pencil beam and (even slight) delay-beam squint effects. Except for the indispensable signal processing, the transceivers of aeronautical communication systems should be elaborately designed to eliminate the delay-beam squint effects and ensure that all carrier frequencies within effective bandwidth can establish a reliable $\mathrm{THz}$ communication link. A common treatment of delay-beam squint effects is to design the transceiver based on the TTDU module [40], [41]. The optimal TTDU module is made up of numerous true-time delay units, and each unit is assigned to its dedicated antenna [42], where the detailed designs of these tunable TTDUs can be found in [43], [44]. Nevertheless, the excessively high hardware complexity and cost of this optimal module prompt us to design a sub-optimal implementation of TTDU module, i.e., GTTDU module based transceiver structure ${ }^{7}$ as shown in Fig. 5. From Fig. 5, we observe that except for the antenna array, this transceiver structure contains a GTTDU module and a reconfigurable RF selection network involving a subconnected PSN and an Antenna Switching Network (ASN) [45], where this ASN can control the active or inactive state of the antenna elements to form different connection patterns

${ }^{7}$ Since the TTDU/GTTDU module is difficult to tackle multiple path signals in the analog domain simultaneously, the proposed transceiver structure and the subsequent solution for $\mathrm{THz}$ aeronautical communications cannot be directly applied in terrestrial vehicular communication scenarios, where the non-LoS components caused by various scatterers are ubiquitous. 


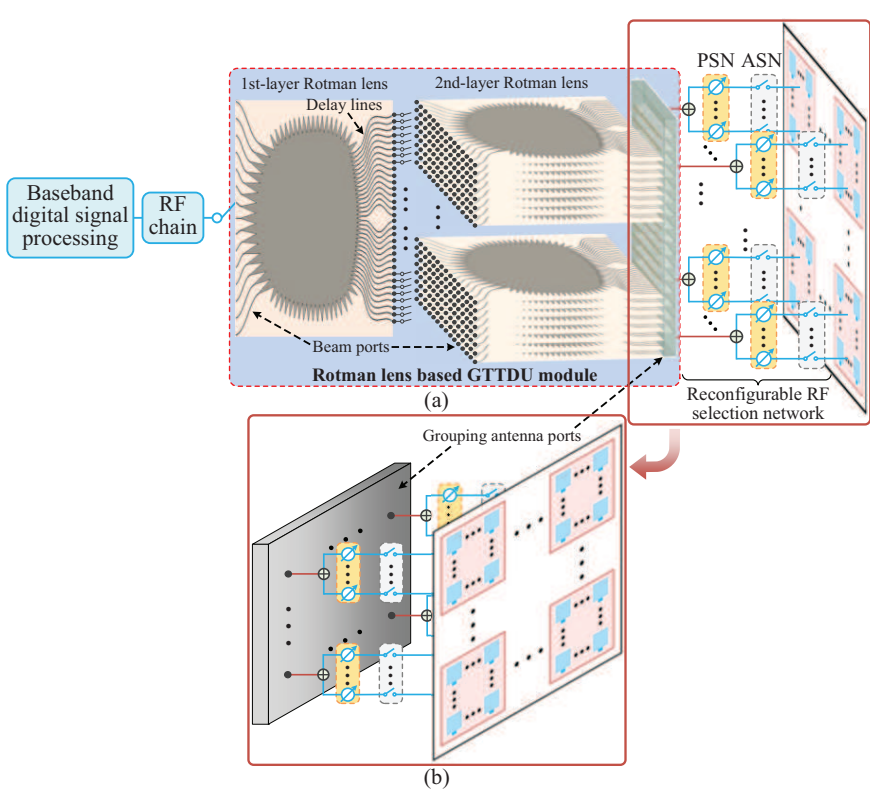

Fig. 6. (a) A feasible transceiver structure corresponding to one RF, where the Rotman lens-based GTTDU module can be utilized to implement the practical tunable TTDU module [46]; and (b) the other side elevation drawing of a part of RF front-end that includes the grouping antenna ports, reconfigurable RF selection network, and THz UM-MIMO array. The beam ports of the first-layer and second-layer Rotman lenses steer the horizontal and vertical directions, respectively. The total number of grouping antenna ports is consistent with that of antenna groups in the previous GTTDU module. This elaborated cascading two-layer Rotman lenses are equivalent to the wideband phase shifters of the tunable TTD module, which can be utilized to eliminate the beam squint effect.

of the RF selection network at the angle estimation stage. In this GTTDU module, a TTDU can be shared by a group of antennas and this imperfect hardware limitation can be handled by the subsequent signal processing algorithms well. Observe that although the delay squint effect for the whole UM array can be non-negligible, this effect for antennas within a group is mild. Hence, the GTTDU module can mitigate the delay squint effect among the antennas in different groups, and the residual phase deviations of these antennas within each group can be further eliminated using their respective phase shifters. Furthermore, to illustrate the feasibility of the transceiver designed in Fig. 5, we propose a potential implementation of transceiver structure involving the Rotman lens-based GTTDU module in Fig. 6, where the cascading two-layer Rotman lenses can be utilized to implement the full-dimensional beamforming [46]. The Rotman lens based GTTDU module is a practical photonic implementation [47], and this design employs the optical properties of electromagnetic waves to achieve the tunable TTD module [48], [49], which provides a prospective direction for our future research work.

When the acquired angle information is accurate enough, the impact of delay squint effect would be significantly mitigated using this GTTDU module. To be specific, based on the prior information acquired from navigation information, the rough estimates of azimuth and elevation angles at BSs (aircraft) can be defined as $\left\{\widetilde{\theta}_{l}^{\mathrm{BS}}\right\}_{l=1}^{L}\left(\left\{\widetilde{\theta}_{l}^{\mathrm{AC}}\right\}_{l=1}^{L}\right)$ and $\left\{\widetilde{\varphi}_{l}^{\mathrm{BS}}\right\}_{l=1}^{L}$ $\left(\left\{\widetilde{\varphi}_{l}^{\mathrm{AC}}\right\}_{l=1}^{L}\right)$, respectively, and the corresponding horizontally and vertically virtual angles are $\left\{\widetilde{\mu}_{l}^{\mathrm{BS}}\right\}_{l=1}^{L}\left(\left\{\widetilde{\mu}_{l}^{\mathrm{AC}}\right\}_{l=1}^{L}\right)$ and
$\left\{\widetilde{\nu}_{l}^{\mathrm{BS}}\right\}_{l=1}^{L}\left(\left\{\widetilde{\nu}_{l}^{\mathrm{AC}}\right\}_{l=1}^{L}\right)$, respectively. According to $\boldsymbol{H}_{\mathrm{DL}, l}^{[n]}[k]$ in (4), we present the expression of the DL channel matrix after ideal TTDU module processing in the following lemma, denoted by $\widetilde{\boldsymbol{H}}_{\mathrm{DL}, l}^{[n]}[k]$, which is proved in Appendix B.

Lemma 1: According to the rough angle estimates above, the antenna transmission delays of THz UM-MIMO arrays at BSs and aircraft can be compensated using the ideal TTDU module, and the compensated DL spatial-frequency channel matrix $\widetilde{\boldsymbol{H}}_{\mathrm{DL}, l}^{[n]}[k]$ can then be formulated as

$$
\begin{aligned}
\widetilde{\boldsymbol{H}}_{\mathrm{DL}, l}^{[n]}[k]= & \sqrt{G_{l}} \alpha_{l} e^{\mathrm{j} 2 \pi \psi_{l, k}(n-1) T_{\mathrm{sym}}} e^{-\mathrm{j} 2 \pi\left(\frac{k-1}{K}-\frac{1}{2}\right) f_{s} \tau_{l}} \\
& \times \widetilde{\boldsymbol{A}}_{\mathrm{DL}, l}[k],
\end{aligned}
$$

in which

$$
\widetilde{\boldsymbol{A}}_{\mathrm{DL}, l}[k]=\boldsymbol{A}_{\mathrm{DL}, l}[k] \circ \underbrace{\left(\overline{\boldsymbol{a}}_{\mathrm{AC}}\left(\widetilde{\mu}_{l}^{\mathrm{AC}}, \widetilde{\nu}_{l}^{\mathrm{AC}}, k\right) \overline{\boldsymbol{a}}_{\mathrm{BS}}^{\mathrm{H}}\left(\widetilde{\mu}_{l}^{\mathrm{BS}}, \widetilde{\nu}_{l}^{\mathrm{BS}}, k\right)\right)}_{\widetilde{\boldsymbol{A}}_{\mathrm{DL}, l}[k]} *
$$

By comparing $\widetilde{\bar{A}}_{\mathrm{DL}, l}[k]$ in (13) and $\overline{\boldsymbol{A}}_{\mathrm{DL}, l}[k]$ in (5), we can find that if we can acquire the perfect angle information, the beam squint effect part can be perfectly eliminated, i.e., $\widetilde{\bar{A}}_{\mathrm{DL}, l}[k]=\overline{\boldsymbol{A}}_{\mathrm{DL}, l}[k]$ and then $\widetilde{\boldsymbol{A}}_{\mathrm{DL}, l}[k]=\boldsymbol{A}_{\mathrm{DL}, l}$ when $\widetilde{\mu}_{l}^{\mathrm{AC}}=\mu_{l}^{\mathrm{AC}}, \widetilde{\nu}_{l}^{\mathrm{AC}}=\nu_{l}^{\mathrm{AC}}, \widetilde{\mu}_{l}^{\mathrm{BS}}=\mu_{l}^{\mathrm{BS}}$, and $\widetilde{\nu}_{l}^{\mathrm{BS}}=\nu_{l}^{\mathrm{BS}}$. Moreover, according to (10) and (11), the compensated UL spatial-frequency channel matrix $\widetilde{\boldsymbol{H}}_{\mathrm{UL}, l}^{[m]}[k]$ has the similar expressions, which are omitted for simplicity.

The ideal TTDU module provides a performance upperbounds for the parameter estimation or data transmission, and we can design the sub-optimal GTTDU module adopted by our solution and the corresponding signal processing algorithms to approach these upper-bounds. The practical DL/UL spatialfrequency channel matrices compensated by the GTTDU module can be derived from (12) and (13). Specifically, all antenna groups for GTTDU module have the same size, i.e., $\widetilde{M}_{\mathrm{BS}}^{\mathrm{h}} \times \widetilde{M}_{\mathrm{BS}}^{\mathrm{v}}$ at BSs and $\widetilde{M}_{\mathrm{AC}}^{\mathrm{h}} \times \widetilde{M}_{\mathrm{AC}}^{\mathrm{v}}$ at aircraft, and the central antenna in each group can be regarded as the benchmark of antenna transmission delay for designing the corresponding TTDU. Moreover, to minimize the beam squint effect caused by antenna grouping as much as possible, the phase deviations of the rest antennas in one group can be compensated using the low-cost PSN, where the phase values at central carrier are treated as the benchmark for calculating these deviations. For convenience, the effective UL and DL channel matrices compensated by the GTTDU module can be also denoted as $\widetilde{\boldsymbol{H}}_{\mathrm{UL}, l}^{[m]}[k]$ and $\widetilde{\boldsymbol{H}}_{\mathrm{DL}, l}^{[n]}[k]$, respectively.

At the initial channel estimation stage, we adopt the Orthogonal Frequency Division Multiple Access (OFDMA) to distinguish the pilot signals transmitted from different BSs and improve the accuracy of the estimated channel parameters. Hence, $K$ subcarriers can be equally assigned to $L \mathrm{BSs}$, where the alternating subcarrier index allocation with equal intervals is adopted and the ordered subcarrier index set assigned to the lth BS is $\mathcal{K}_{l}$ with $K_{l}=\left|\mathcal{K}_{l}\right|_{c}$. Moreover, the azimuth/elevation angles at BSs can be estimated in UL, while the rest of channel parameters are acquired in DL. 


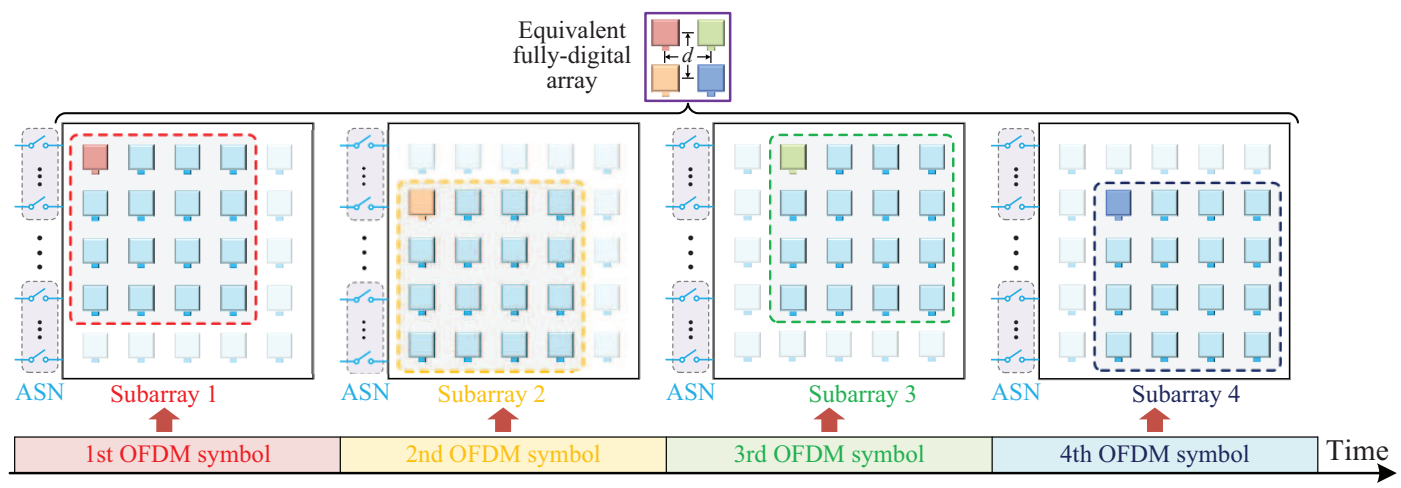

Fig. 7. The schematic diagram of subarray selection at the initial angle estimation stage, where the different antenna connection patterns can be formed by controlling the ASN of the reconfigurable RF selection network. Taking the UPA of size $5 \times 5$ as an example, this UPA can be partitioned into 4 subarrays of size $4 \times 4$, and the interval between each subarray is the width of one antenna. The same RF chain sequentially selects the corresponding subarrays in 4 successive OFDM symbols to receive signals, and these received signals will be equivalent to the signals received by a low-dimensional fully-digital array of size $2 \times 2$ with the critical antenna spacing $d$.

\section{A. Fine Angle Estimation Based on Reconfigurable RF Selec- tion Network}

1) Fine Angle Estimation at BSs: Due to the insufficient valid observation caused by the limited number of RF chains at the BSs, it is necessary to accumulate multiple OFDM symbols in the time domain to estimate the angles. To mitigate the inter-carrier interference within one OFDM symbol caused by the large Doppler shifts, the acquired rough Doppler shift estimates are first utilized to compensate the transmitted signals, so that the compensated channels of multiple OFDM symbols can be slow time-varying. By transforming the different RF connection pattern of antenna array, we observe a fact that the received signals adopting different selected subarrays only differ by one envisaged phase value if the transceiver has the same configuration, and those regular phase differences can construct the array response vector of lowdimensional fully-digital array. Taking the UPA with size of $5 \times 5$ in Fig. 7 as an example, we can select 4 subarrays of size $4 \times 4$ in 4 successive OFDM symbols to form the array response vector of equivalent fully-digital array with size of $2 \times 2$ by controlling the reconfigurable RF selection network. Specifically, we intend to use $I_{\mathrm{BS}}$ OFDM symbols to estimate the angles at BSs, where each OFDM symbol adopts a dedicated RF connection pattern (i.e., the selected subarray). By employing the rough angle estimates at aircraft and BSs, the analog precoding and combining vectors, i.e., $\boldsymbol{p}_{\mathrm{RF}, l}$ and $\boldsymbol{q}_{\mathrm{RF}, l}^{[m]}$ for $1 \leq l \leq L, 1 \leq m \leq I_{\mathrm{BS}}$, can be first designed. In terms of $\boldsymbol{p}_{\mathrm{RF}, l}$, initialize $\boldsymbol{p}_{\mathrm{RF}, l}$ as $\boldsymbol{p}_{\mathrm{RF}, l}=\mathbf{0}_{N_{\mathrm{AC}}}$, and then let $\left[\boldsymbol{p}_{\mathrm{RF}, l}\right]_{\mathcal{I}_{\mathrm{AC}, l}}=\frac{1}{\sqrt{M_{\mathrm{AC}}}}\left[\boldsymbol{a}_{\mathrm{AC}}\left(\widetilde{\mu}_{l}^{\mathrm{AC}}, \widetilde{\nu}_{l}^{\mathrm{AC}}\right)\right]_{\mathcal{I}_{\mathrm{AC}, l}}$. Here $\mathcal{I}_{\mathrm{AC}, l}$ with $M_{\mathrm{AC}}=\left|\mathcal{I}_{\mathrm{AC}, l}\right|_{c}$ denotes the antenna index of subarray assigned to the $l$ th BS, since each subarray at aircraft only communicates with its corresponding BS as shown in Fig. 4(a). To design $\left\{\boldsymbol{q}_{\mathrm{RF}, l}^{[m]}\right\}_{m=1}^{I_{\mathrm{BS}}}$, the UM-MIMO array at BS can be partitioned into $I_{\mathrm{BS}}=I_{\mathrm{BS}}^{\mathrm{h}} I_{\mathrm{BS}}^{\mathrm{v}}$ smaller subarrays to yield the array response vector of equivalent low-dimensional fully-digital array with size of $I_{\mathrm{BS}}^{\mathrm{h}} \times I_{\mathrm{BS}}^{\mathrm{v}}$, where the sizes of these smaller subarrays are $\bar{M}_{\mathrm{BS}}^{\mathrm{h}} \times \bar{M}_{\mathrm{BS}}^{\mathrm{v}}\left(\bar{M}_{\mathrm{BS}}^{\mathrm{h}}=N_{\mathrm{BS}}^{\mathrm{h}}-I_{\mathrm{BS}}^{\mathrm{h}}+1\right.$ and $\left.\bar{M}_{\mathrm{BS}}^{\mathrm{v}}=N_{\mathrm{BS}}^{\mathrm{v}}-I_{\mathrm{BS}}^{\mathrm{v}}+1\right)$ and their number of antennas is $\bar{M}_{\mathrm{BS}}=\bar{M}_{\mathrm{BS}}^{\mathrm{h}} \bar{M}_{\mathrm{BS}}^{\mathrm{v}}$. Defining $m=\left(i_{\mathrm{BS}}^{\mathrm{v}}-1\right) I_{\mathrm{BS}}^{\mathrm{h}}+i_{\mathrm{BS}}^{\mathrm{h}}$ with $i_{\mathrm{BS}}^{\mathrm{h}}$ and $i_{\mathrm{BS}}^{\mathrm{v}}$ being the $\left(i_{\mathrm{BS}}^{\mathrm{h}}, i_{\mathrm{BS}}^{\mathrm{v}}\right)$ th subarray for $1 \leq i_{\mathrm{BS}}^{\mathrm{h}} \leq I_{\mathrm{BS}}^{\mathrm{h}}$ and $1 \leq i_{\mathrm{BS}}^{\mathrm{v}} \leq I_{\mathrm{BS}}^{\mathrm{v}}$, respectively, the antenna index of the selected $m$ th subarray that corresponds to the $m$ th OFDM symbol can be denoted by $\mathcal{I}_{\mathrm{BS}}^{[m]}$ with $\bar{M}_{\mathrm{BS}}=\left|\mathcal{I}_{\mathrm{BS}}^{[m]}\right|_{c}$, so that $\boldsymbol{q}_{\mathrm{RF}, l}^{[m]}$ can be also initialized as $\boldsymbol{q}_{\mathrm{RF}, l}^{[m]}=\mathbf{0}_{N_{\mathrm{BS}}}$, and then let $\left[\boldsymbol{q}_{\mathrm{RF}, l}^{[m]}\right]_{\mathcal{I}_{\mathrm{BS}}^{[m]}}=\frac{1}{\sqrt{\bar{M}_{\mathrm{BS}}}}\left[\boldsymbol{a}_{\mathrm{BS}}\left(\widetilde{\mu}_{l}^{\mathrm{BS}}, \widetilde{\nu}_{l}^{\mathrm{BS}}\right)\right]_{\mathcal{I}_{\mathrm{BS}}^{[1]}}$ for $1 \leq m \leq I_{\mathrm{BS}}$.

According to the UL transmission model in (1), the received signal $y_{\mathrm{UL}, l}^{[m]}\left[k_{l}\right]$ at the $k_{l}$ th subcarrier of the $m$ th OFDM symbol transmitted by the $l$ th BS can be expressed as

$y_{\mathrm{UL}, l}^{[m]}\left[k_{l}\right]=\sqrt{P_{l}}\left(\boldsymbol{q}_{\mathrm{RF}, l}^{[m]}\right)^{\mathrm{H}} \widetilde{\boldsymbol{H}}_{\mathrm{UL}, l}^{[m]}\left[k_{l}\right] \boldsymbol{p}_{\mathrm{RF}, l} s_{\mathrm{UL}, l}^{[m]}\left[k_{l}\right]+n_{\mathrm{UL}, l}^{[m]}\left[k_{l}\right]$,

where $k_{l} \in \mathcal{K}_{l}, 1 \leq m \leq I_{\mathrm{BS}}, \widetilde{\boldsymbol{H}}_{\mathrm{UL}, l}^{[m]}\left[k_{l}\right]$ is the channel matrix compensated by GTTDU module and rough Doppler shift estimates, and $s_{\mathrm{UL}, l}^{[m]}\left[k_{l}\right]$ and $n_{\mathrm{UL}, l}^{[m]}\left[k_{l}\right]$ are the transmitted pilot signal and noise, respectively. By collecting the received signals at $K_{l}$ subcarriers as $\boldsymbol{y}_{\mathrm{UL}, l}^{[m]} \in \mathbb{C}^{K_{l}}$ and substituting the UL channel matrix in (10) into $\boldsymbol{y}_{\mathrm{UL}, l}^{[m]}$, we have

$$
\begin{aligned}
\boldsymbol{y}_{\mathrm{UL}, l}^{[m]}= & {\left[y_{\mathrm{UL}, l}^{[m]}\left[\left\{\mathcal{K}_{l}\right\}_{1}\right] \cdots y_{\mathrm{UL}, l}^{[m]}\left[\left\{\mathcal{K}_{l}\right\}_{K_{l}}\right]\right]^{\mathrm{T}} } \\
= & \sqrt{P_{l} G_{l}} \alpha_{l}\left(\boldsymbol{q}_{\mathrm{RF}, l}^{[m]}\right)^{\mathrm{H}} \boldsymbol{A}_{\mathrm{UL}, l} \boldsymbol{p}_{\mathrm{RF}, l} \boldsymbol{s}_{\mathrm{UL}, l}^{[m]} \circ \widetilde{\boldsymbol{y}}_{\mathrm{UL}, l}^{[m]} \\
& +\boldsymbol{n}_{\mathrm{UL}, l}^{[m]},
\end{aligned}
$$

where $\boldsymbol{s}_{\mathrm{UL}, l}^{[m]}=\left[s_{\mathrm{UL}, l}^{[m]}\left[\left\{\mathcal{K}_{l}\right\}_{1}\right] \cdots s_{\mathrm{UL}, l}^{[m]}\left[\left\{\mathcal{K}_{l}\right\}_{K_{l}}\right]\right]^{\mathrm{T}} \in \mathbb{C}^{K_{l}}, \widetilde{\boldsymbol{y}}_{\mathrm{UL}, l}^{[m]}$ is the error vector including the residual beam squint caused by inaccurate prior information, and $\boldsymbol{n}_{\mathrm{UL}, l}^{[\mathrm{m}]}$ is the corresponding noise vector. Moreover, the same transmitted pilot signals are adopted for $I_{\mathrm{BS}}$ OFDM symbol, i.e., $s_{\mathrm{UL}, l}\left[k_{l}\right]=s_{\mathrm{UL}, l}^{[m]}\left[k_{l}\right]$, and accordingly, $\boldsymbol{s}_{\mathrm{UL}, l}=s_{\mathrm{UL}, l}^{[m]}$ for $1 \leq m \leq I_{\mathrm{BS}}$. Taking the transposition of $\left\{\boldsymbol{y}_{\mathrm{UL}, l}^{[m]}\right\}_{m=1}^{I_{\mathrm{BS}}}$ received from $I_{\mathrm{BS}}$ OFDM symbols, we can stack them as $\boldsymbol{Y}_{\mathrm{UL}, l}=\left[\boldsymbol{y}_{\mathrm{UL}, l}^{[1]} \cdots \boldsymbol{y}_{\mathrm{UL}, l}^{\left[I_{\mathrm{BS}}\right]}\right]^{\mathrm{T}} \in \mathbb{C}^{I_{\mathrm{BS}} \times K_{l}}$, i.e.,

$$
\begin{aligned}
\boldsymbol{Y}_{\mathrm{UL}, l}= & \sqrt{P_{l} G_{l}} \alpha_{l}\left(\boldsymbol{Q}_{\mathrm{RF}, l}^{\mathrm{H}} \boldsymbol{A}_{\mathrm{UL}, l} \boldsymbol{p}_{\mathrm{RF}, l} \boldsymbol{s}_{\mathrm{UL}, l}^{\mathrm{T}}\right) \circ \widetilde{\boldsymbol{Y}}_{\mathrm{UL}, l} \\
& +\boldsymbol{N}_{\mathrm{UL}, l},
\end{aligned}
$$


where $\boldsymbol{Q}_{\mathrm{RF}, l}=\left[\boldsymbol{q}_{\mathrm{RF}, l}^{[1]} \cdots \boldsymbol{q}_{\mathrm{RF}, l}^{\left[I_{\mathrm{BS}}\right]}\right] \in \mathbb{C}^{N_{\mathrm{BS}} \times I_{\mathrm{BS}}}$ and $\tilde{\boldsymbol{Y}}_{\mathrm{UL}, l}=$ $\left[\widetilde{\boldsymbol{y}}_{\mathrm{UL}, l}^{[1]} \cdots \widetilde{\boldsymbol{y}}_{\mathrm{UL}, l}^{\left[I_{\mathrm{BS}}\right]}\right]$ are the analog combining and residual beam squint matrices, respectively, and $\boldsymbol{N}_{\mathrm{UL}, l}$ is the noise matrix. By utilizing this analog combining matrix $Q_{\mathrm{RF}, l}$, the array response vector of equivalent low-dimensional fullydigital array can be formed to estimate the angles at BSs using array signal processing techniques. To be specific, compared with $\left(\boldsymbol{q}_{\mathrm{RF}, l}^{[1]}\right)^{\mathrm{H}} \boldsymbol{a}_{\mathrm{BS}}\left(\mu_{l}^{\mathrm{BS}}, \nu_{l}^{\mathrm{BS}}\right)$ for $m=1$ in (15), $\left(\boldsymbol{q}_{\mathrm{RF}, l}^{[m]}\right)^{\mathrm{H}} \boldsymbol{a}_{\mathrm{BS}}\left(\mu_{l}^{\mathrm{BS}}, \nu_{l}^{\mathrm{BS}}\right)$ is multiplied by an extra phase shift $e^{\mathrm{j}\left(\left(i_{\mathrm{BS}}^{\mathrm{h}}-1\right) \mu_{l}^{\mathrm{BS}}+\left(i_{\mathrm{BS}}^{\mathrm{v}}-1\right) \nu_{l}^{\mathrm{BS}}\right)}$ for $m=\left(i_{\mathrm{BS}}^{\mathrm{v}}-1\right) I_{\mathrm{BS}}^{\mathrm{h}}+i_{\mathrm{BS}}^{\mathrm{h}}$ and $2 \leq m \leq I_{\mathrm{BS}}$. Obviously, these regular phase shifts can constitute the effective array response vector of equivalent fully-digital array with size of $I_{\mathrm{BS}}^{\mathrm{h}} \times I_{\mathrm{BS}}^{\mathrm{v}}$, i.e., $\overline{\overline{\boldsymbol{a}}}_{\mathrm{BS}}\left(\mu_{l}^{\mathrm{BS}}, \nu_{l}^{\mathrm{BS}}\right)=$ $\boldsymbol{a}_{\mathrm{v}}\left(\nu_{l}^{\mathrm{BS}}, I_{\mathrm{BS}}^{\mathrm{v}}\right) \otimes \boldsymbol{a}_{\mathrm{h}}\left(\mu_{l}^{\mathrm{BS}}, I_{\mathrm{BS}}^{\mathrm{h}}\right) \in \mathbb{C}^{I_{\mathrm{BS}}}$. Thus, the UL received signal matrix $\boldsymbol{Y}_{\mathrm{UL}, l}$ in (16) can be then rewritten as

$$
\boldsymbol{Y}_{\mathrm{UL}, l}=\gamma_{\mathrm{UL}, l}\left(\overline{\overline{\boldsymbol{a}}}_{\mathrm{BS}}\left(\mu_{l}^{\mathrm{BS}}, \nu_{l}^{\mathrm{BS}}\right) \boldsymbol{s}_{\mathrm{UL}, l}^{\mathrm{T}}\right) \circ \tilde{\boldsymbol{Y}}_{\mathrm{UL}, l}+\boldsymbol{N}_{\mathrm{UL}, l},
$$

where $\gamma_{\mathrm{UL}, l}=\sqrt{P_{l} G_{l}} \alpha_{l}\left(\boldsymbol{q}_{\mathrm{RF}, l}^{[1]}\right)^{\mathrm{H}} \boldsymbol{A}_{\mathrm{UL}, l} \boldsymbol{p}_{\mathrm{RF}, l}$ is the beamaligned effective channel gain.

For the received signal model in (17), we propose a prioraided iterative angle estimation algorithm as follows. At the first iteration, i.e., $i_{\mathrm{BS}}=1$, the azimuth and elevation angles at the $l$ th BS can be first estimated as $\widehat{\theta}_{l}^{\left(i_{\mathrm{BS}}\right)}$ and $\widehat{\varphi}_{l}^{\left(i_{\mathrm{BS}}\right)}$, and the corresponding horizontally and vertically virtual angles are $\widehat{\mu}_{l}^{\left(i_{\mathrm{BS}}\right)}$ and $\widehat{\nu}_{l}^{\left(i_{\mathrm{BS}}\right)}$ for $1 \leq l \leq L$ by applying the TDUESPRIT algorithm [36], [37] to the received signal matrix $\boldsymbol{Y}_{\mathrm{UL}, l}$. Furthermore, to minimize the impact of $\widetilde{\boldsymbol{Y}}_{\mathrm{UL}, l}$ on (17), more accurate angle estimates can be acquired by utilizing the estimated angles above to iteratively compensate $\boldsymbol{Y}_{\mathrm{UL}, l}$ at the subsequent iterations (i.e., $i_{\mathrm{BS}} \geq 2$ ). Specifically, for the $i_{\mathrm{BS}}$ th iteration, according to the rough virtual angle estimates $\widetilde{\mu}_{l}^{\mathrm{BS}}$ and $\widetilde{\nu}_{l}^{\mathrm{BS}}$, and $\widehat{\mu}_{l}^{\left(i_{\mathrm{BS}}-1\right)}$ and $\widehat{\nu}_{l}^{\left(i_{\mathrm{BS}}-1\right)}$ estimated at the $\left(i_{\mathrm{BS}}-1\right)$ th iteration, we define the compensation matrix as $\widetilde{\boldsymbol{Y}}_{\mathrm{UL}, l}^{\left(i_{\mathrm{BS}}-1\right)}=\left[\widetilde{\boldsymbol{y}}_{\mathrm{UL}, l}^{\left(i_{\mathrm{BS}}-1\right)}\left[\left\{\mathcal{K}_{l}\right\}_{1}\right] \cdots \widetilde{\boldsymbol{y}}_{\mathrm{UL}, l}^{\left(i_{\mathrm{BS}}-1\right)}\left[\left\{\mathcal{K}_{l}\right\}_{K_{l}}\right]\right]$, whose the $k_{l}$ th column $\widetilde{\boldsymbol{y}}_{\mathrm{UL}, l}^{\left(i_{\mathrm{BS}}-1\right)}\left[k_{l}\right] \in \mathbb{C}^{I_{\mathrm{BS}}}$ is given by

$$
\begin{aligned}
& \widetilde{\boldsymbol{y}}_{\mathrm{UL}, l}^{\left(i_{\mathrm{BS}}-1\right)}\left[k_{l}\right] \\
& =\left(\overline{\boldsymbol{a}}_{\mathrm{v}}\left(\widetilde{\nu}_{l}^{\mathrm{BS}}, I_{\mathrm{BS}}^{\mathrm{v}}, k_{l}\right) \otimes \overline{\boldsymbol{a}}_{\mathrm{h}}\left(\widetilde{\mu}_{l}^{\mathrm{BS}}, I_{\mathrm{BS}}^{\mathrm{h}}, k_{l}\right)\right)^{*} \\
& \quad \circ\left(\overline{\boldsymbol{a}}_{\mathrm{v}}\left(\widehat{\nu}_{l}^{\left(i_{\mathrm{BS}}-1\right)}, I_{\mathrm{BS}}^{\mathrm{v}}, k_{l}\right) \otimes \overline{\boldsymbol{a}}_{\mathrm{h}}\left(\widehat{\mu}_{l}^{\left(i_{\mathrm{BS}}-1\right)}, I_{\mathrm{BS}}^{\mathrm{h}}, k_{l}\right)\right) .
\end{aligned}
$$

After the compensation matrix $\tilde{\boldsymbol{Y}}_{\mathrm{UL}, l}^{\left(i_{\mathrm{BS}}-1\right)}$ processing, the processed matrix $\boldsymbol{Y}_{\mathrm{UL}, l}^{\left(i_{\mathrm{BS}}\right)}=\left(\widetilde{\boldsymbol{Y}}_{\mathrm{UL}, l}^{\left(i_{\mathrm{BS}}-1\right)}\right)^{*} \circ \boldsymbol{Y}_{\mathrm{UL}, l}$ can be written as

$$
\begin{aligned}
\boldsymbol{Y}_{\mathrm{UL}, l}^{\left(i_{\mathrm{BS}}\right)}= & \gamma_{\mathrm{UL}, l}\left(\overline{\overline{\boldsymbol{a}}}_{\mathrm{BS}}\left(\mu_{l}^{\mathrm{BS}}, \nu_{l}^{\mathrm{BS}}\right) \boldsymbol{s}_{\mathrm{UL}, l}^{\mathrm{T}}\right) \\
& \circ\left(\widetilde{\boldsymbol{Y}}_{\mathrm{UL}, l} \circ\left(\widetilde{\boldsymbol{Y}}_{\mathrm{UL}, l}^{\left(i_{\mathrm{BS}}-1\right)}\right)^{*}\right)+\boldsymbol{N}_{\mathrm{UL}, l}^{\left(i_{\mathrm{BS}}\right),}
\end{aligned}
$$

where $\boldsymbol{N}_{\mathrm{UL}, l}^{\left(i_{\mathrm{BS}}\right)}$ is the processed noise matrix. By applying the TDU-ESPRIT algorithm to those matrices $\left\{\boldsymbol{Y}_{\mathrm{UL}, l}^{\left(i_{\mathrm{BS}}\right)}\right\}_{l=1}^{L}$ again, we can obtain the more accurate angle estimates until the maximum number of iterations $i_{\mathrm{BS}}^{\max }$ is reached, i.e., $i_{\mathrm{BS}}=i_{\mathrm{BS}}^{\max }$. Finally, the estimates of azimuth and elevation angles and the corresponding virtual angles at BSs can be denoted as $\widehat{\theta}_{l}^{\mathrm{BS}}=\widehat{\theta}_{l}^{\left(i_{\mathrm{BS}}^{\max }\right)}, \widehat{\varphi}_{l}^{\mathrm{BS}}=\widehat{\varphi}_{l}^{\left(i_{\mathrm{BS}}^{\mathrm{max}}\right)}, \widehat{\mu}_{l}^{\mathrm{BS}}=\widehat{\mu}_{l}^{\left(i_{\mathrm{BS}}^{\max }\right)}$,

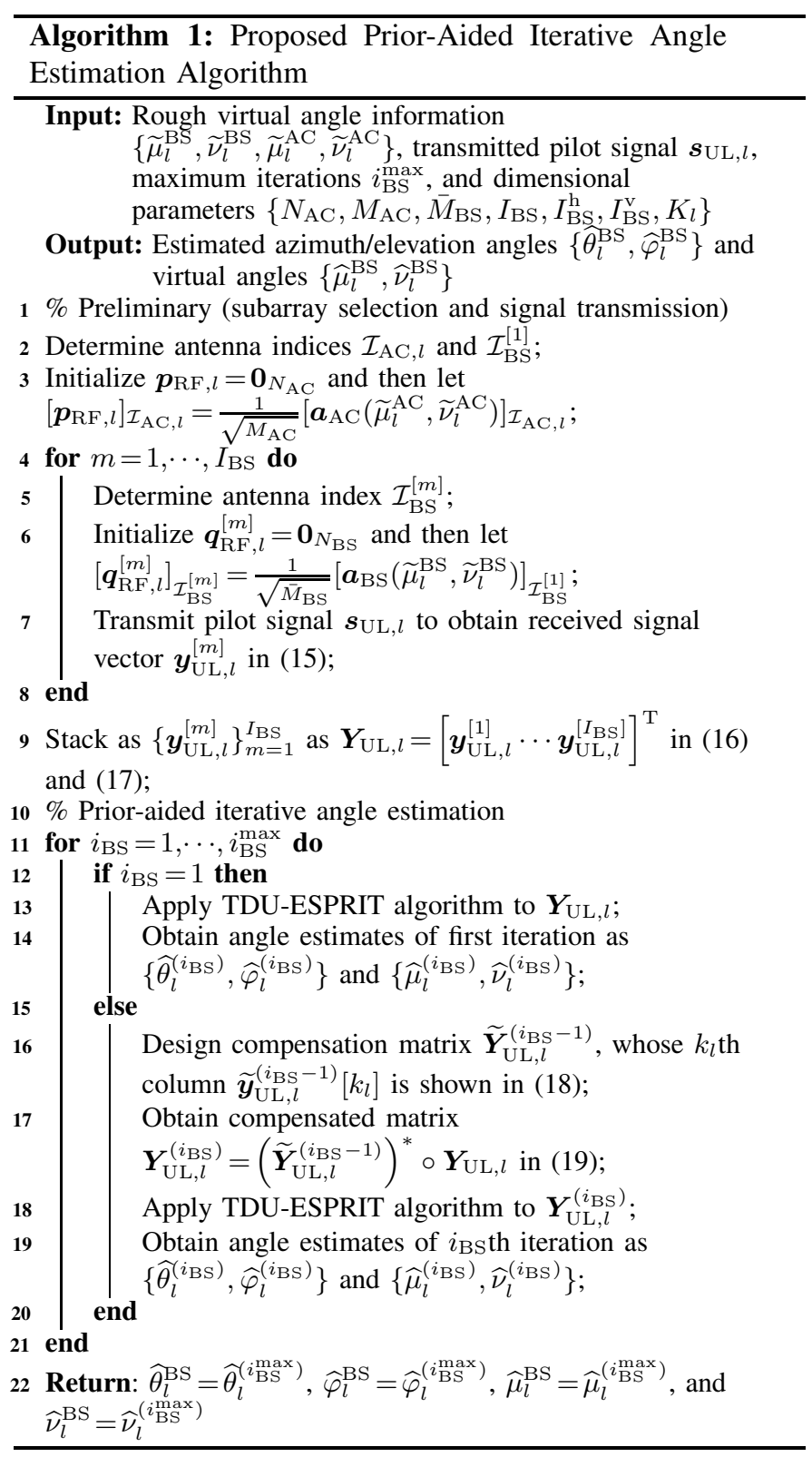

and $\widehat{\nu}_{l}^{\mathrm{BS}}=\widehat{\nu}_{l}^{\left(i_{\mathrm{BS}}^{\max }\right)}$ for $1 \leq l \leq L$. The proposed prior-aided iterative angle estimation algorithm above is summarized in Algorithm 1, where the beam squint effect can be addressed well.

Remark 1: Based on the analysis above, by controlling the connection patterns, the reconfigurable RF selection network can select the desired subarrays to obtain an equivalent lowdimensional fully-digital array, so that the robust array signal processing techniques can be utilized to obtain the accurate angle estimates. On the other hand, the size of each selected subarray, i.e., $\bar{M}_{\mathrm{BS}}^{\mathrm{h}} \times \bar{M}_{\mathrm{BS}}^{\mathrm{v}}$, is large enough. This indicates that at the initial angle estimation stage, we can achieve the sufficient full-dimensional beamforming gain with the aid of rough angle estimates to effectively combat the severe path loss of long-distance THz links and improve the receive SNR.

2) Fine Angle Estimation at Aircraft: Due to the channel reciprocity of UL and DL, the acquisition of fine angle estimates at aircraft in DL is similar to the fine angle estimation at 
BSs. At this stage, instead of using the rough angle estimates, the fine angles estimated at BSs in Section III-A1 can be used not only to design the analog precoding vectors at BSs for beam alignment with improved receive SNR, but also to refine the GTTDU modules at BSs. Specifically, we consider $I_{\mathrm{AC}}=I_{\mathrm{AC}}^{\mathrm{h}} I_{\mathrm{AC}}^{\mathrm{V}}$ OFDM symbols to estimate the fine azimuth and elevation angles at aircraft, where the size of the equivalent low-dimensional fully-digital array is $I_{\mathrm{AC}}^{\mathrm{h}} \times I_{\mathrm{AC}}^{\mathrm{v}}$. Based on the estimated $\left\{\widehat{\mu}_{l}^{\mathrm{BS}}, \widehat{\nu}_{l}^{\mathrm{BS}}\right\}_{l=1}^{L}$, the analog precoding vector can be designed as $\boldsymbol{f}_{\mathrm{RF}, l}=\boldsymbol{a}_{\mathrm{BS}}\left(\widehat{\mu}_{l}^{\mathrm{BS}}, \widehat{\nu}_{l}^{\mathrm{BS}}\right)$ for $1 \leq l \leq L$. By employing the reconfigurable RF selection network, the selected antenna index in the $n$th OFDM sysmbol at the $l$ th aircraft subarray is denoted by $\mathcal{I}_{\mathrm{AC}, l}^{[n]}$ with $\bar{M}_{\mathrm{AC}}=\left|\mathcal{I}_{\mathrm{AC}, l}^{[n]}\right|_{c}$. Then, initialize the analog combining vector as $\boldsymbol{w}_{\mathrm{RF}, l}^{[n]}=\mathbf{0}_{N_{\mathrm{AC}}}$, and then let $\left[\boldsymbol{w}_{\mathrm{RF}, l}^{[n]}\right]_{\mathcal{I}_{\mathrm{AC}, l}^{[n]}}=\frac{1}{\sqrt{\bar{M}_{\mathrm{AC}}}}\left[\boldsymbol{a}_{\mathrm{AC}}\left(\widetilde{\mu}_{l}^{\mathrm{AC}}, \widetilde{\nu}_{l}^{\mathrm{AC}}\right)\right]_{\mathcal{I}_{\mathrm{AC}, l}^{[1]}}$, for $1 \leq n \leq I_{\mathrm{AC}}, 1 \leq l \leq L$.

According to the DL transmission in (2), at the $l$ th RF chain of aircraft, the received signal $y_{\mathrm{DL}, l}^{[n]}\left[k_{l}\right]$ at the $k_{l}$ th subcarrier of the $n$th OFDM symbol corresponding to the $l$ th BS can be expressed as

$y_{\mathrm{DL}, l}^{[n]}\left[k_{l}\right]=\sqrt{P_{l}}\left(\boldsymbol{w}_{\mathrm{RF}, l}^{[n]}\right)^{\mathrm{H}} \widetilde{\boldsymbol{H}}_{\mathrm{DL}, l}^{[n]}\left[k_{l}\right] \boldsymbol{f}_{\mathrm{RF}, l} s_{\mathrm{DL}, l}^{[n]}\left[k_{l}\right]+n_{\mathrm{DL}, l}^{[n]}\left[k_{l}\right]$,

where $k_{l} \in \mathcal{K}_{l}, 1 \leq n \leq I_{\mathrm{AC}}, \widetilde{\boldsymbol{H}}_{\mathrm{DL}, l}^{[n]}\left[k_{l}\right]$ is the compensated DL channel matrix, and $s_{\mathrm{DL}, l}^{[n]}\left[k_{l}\right]$ and $n_{\mathrm{DL}, l}^{[n]}\left[k_{l}\right]$ are the transmitted pilot signal and noise, respectively. Considering the received signals at $K_{l}$ subcarriers of $I_{\mathrm{AC}}$ OFDM symbols, we can obtain the DL received signal matrix $\boldsymbol{Y}_{\mathrm{DL}, l} \in \mathbb{C}^{I_{\mathrm{AC}} \times K_{l}}$ as

$$
\begin{aligned}
& \boldsymbol{Y}_{\mathrm{DL}, l} \\
& \left.=\sqrt{P_{l} G_{l}} \alpha_{l} e^{\mathrm{j} \pi f_{s} \tau_{l}}(\overline{\boldsymbol{W}}_{\mathrm{RF}, l}^{\mathrm{H}} \boldsymbol{A}_{\mathrm{DL}, l} \boldsymbol{f}_{\mathrm{RF}, l} \underbrace{\left(\boldsymbol{a}_{\tau}\left(\mu_{l}^{\tau}, K_{l}\right) \circ \boldsymbol{s}_{\mathrm{DL}, l}\right)}_{\overline{\boldsymbol{s}}_{\mathrm{DL}, l}})^{\mathrm{T}}\right) \\
& \circ \tilde{\boldsymbol{Y}}_{\mathrm{DL}, l}+\boldsymbol{N}_{\mathrm{DL}, l},
\end{aligned}
$$

where $\overline{\boldsymbol{W}}_{\mathrm{RF}, l}=\left[\boldsymbol{w}_{\mathrm{RF}, l}^{[1]} \cdots \boldsymbol{w}_{\mathrm{RF}, l}^{\left[I_{\mathrm{AC}}\right]}\right] \in \mathbb{C}^{N_{\mathrm{AC} \times I_{\mathrm{AC}}}}$ and $\tilde{\boldsymbol{Y}}_{\mathrm{DL}, l}$ are the analog combining and residual beam squint matrices, respectively, $\boldsymbol{s}_{\mathrm{DL}, l}=s_{\mathrm{DL}, l}^{[n]}=$ $\left[s_{\mathrm{DL}, l}^{[n]}\left[\left\{\mathcal{K}_{l}\right\}_{1}\right] \cdots s_{\mathrm{DL}, l}^{[n]}\left[\left\{\mathcal{K}_{l}\right\}_{K_{l}}\right]\right]^{\mathrm{T}} \in \mathbb{C}^{K_{l}}$ for $1 \leq n \leq I_{\mathrm{AC}}$, and $\boldsymbol{N}_{\mathrm{DL}, l}$ is the corresponding noise matrix. In (21), the steering vector associated with path delay $\tau_{l}$ can be defined as $\boldsymbol{a}_{\tau}\left(\mu_{l}^{\tau}, K_{l}\right)=\left[e^{\mathrm{j}\left(\left\{\mathcal{K}_{l}\right\}_{1}-1\right) \mu_{l}^{\tau}} e^{\mathrm{j}\left(\left\{\mathcal{K}_{l}\right\}_{2}-1\right) \mu_{l}^{\tau}} \cdots e^{\mathrm{j}\left(\left\{\mathcal{K}_{l}\right\}_{K_{l}}-1\right) \mu_{l}^{\tau}}\right]^{\mathrm{T}}$ with $\mu_{l}^{\tau}=-2 \pi f_{s} \tau_{l} / K$ being the virtual delay. Similar to (17), $\boldsymbol{Y}_{\mathrm{DL}, l}$ can be rewritten as

$$
\boldsymbol{Y}_{\mathrm{DL}, l}=\gamma_{\mathrm{DL}, l}\left(\overline{\overline{\boldsymbol{a}}}_{\mathrm{AC}}\left(\mu_{l}^{\mathrm{AC}}, \nu_{l}^{\mathrm{AC}}\right) \overline{\boldsymbol{s}}_{\mathrm{DL}, l}^{\mathrm{T}}\right) \circ \widetilde{\boldsymbol{Y}}_{\mathrm{DL}, l}+\boldsymbol{N}_{\mathrm{DL}, l},
$$

where $\gamma_{\mathrm{DL}, l}=\sqrt{P_{l} G_{l}} \alpha_{l} e^{\mathrm{j} \pi f_{s} \tau_{l}}\left(\boldsymbol{w}_{\mathrm{RF}, l}^{[1]}\right)^{\mathrm{H}} \boldsymbol{A}_{\mathrm{DL}, l} \boldsymbol{f}_{\mathrm{RF}, l}$, and $\overline{\overline{\boldsymbol{a}}}_{\mathrm{AC}}\left(\mu_{l}^{\mathrm{AC}}, \nu_{l}^{\mathrm{AC}}\right)=\boldsymbol{a}_{\mathrm{v}}\left(\nu_{l}^{\mathrm{AC}}, I_{\mathrm{AC}}^{\mathrm{v}}\right) \otimes \boldsymbol{a}_{\mathrm{h}}\left(\mu_{l}^{\mathrm{AC}}, I_{\mathrm{AC}}^{\mathrm{h}}\right) \in \mathbb{C}^{I_{\mathrm{AC}}}$ is the effective array response vector of equivalent low-dimensional fully-digital array at the $l$ th subarray of aircraft. For the received signal model in (22), we can also utilize the proposed prior-aided iterative angle estimation algorithm in Algorithm 1 to obtain the more accurate angle estimates. By replacing the input parameters $\left\{\widetilde{\mu}_{l}^{\mathrm{BS}}, \widetilde{\nu}_{l}^{\mathrm{BS}},\left\{\mathcal{I}_{\mathrm{BS}}^{[m]}\right\}_{m=1}^{I_{\mathrm{BS}}}, \boldsymbol{s}_{\mathrm{UL}, l}, \bar{M}_{\mathrm{BS}}, I_{\mathrm{BS}}, I_{\mathrm{BS}}^{\mathrm{h}}, I_{\mathrm{BS}}^{\mathrm{v}}, i_{\mathrm{BS}}, i_{\mathrm{BS}}^{\max }\right\}$ for $\mathrm{BSs}$ with the corresponding parameters $\left\{\widehat{\mu}_{l}^{\mathrm{BS}}, \widehat{\nu}_{l}^{\mathrm{BS}},\left\{\mathcal{I}_{\mathrm{AC}, l}^{[n]}\right\}_{n=1}^{I_{\mathrm{AC}}}, \boldsymbol{s}_{\mathrm{DL}, l}, \bar{M}_{\mathrm{AC}}, I_{\mathrm{AC}}, I_{\mathrm{AC}}^{\mathrm{h}}, I_{\mathrm{AC}}^{\mathrm{v}}, i_{\mathrm{AC}}, i_{\mathrm{AC}}^{\max }\right\}$ for aircraft, the estimates of azimuth and elevation angles and the corresponding virtual angles at aircraft can be obtained as $\widehat{\theta}_{l}^{\mathrm{AC}}=\widehat{\theta}_{l}^{\left(i_{\mathrm{AC}}^{\max }\right)}, \widehat{\varphi}_{l}^{\mathrm{AC}}=\widehat{\varphi}_{l}^{\left(i_{\mathrm{AC}}^{\max }\right)}, \widehat{\mu}_{l}^{\mathrm{AC}}=\widehat{\mu}_{l}^{\left(i_{\mathrm{AC}}^{\max }\right)}$, and $\widehat{\nu}_{l}^{\mathrm{AC}}=\widehat{\nu}_{l}^{\left(i_{\mathrm{AC}}^{\max }\right)}$ for $1 \leq l \leq L$.

\section{B. Fine Doppler Shift Estimation under Doppler-Squint Effect}

Based on the fine angle estimates above, the analog combining vectors of $L$ subarrays at aircraft are designed to achieve beam alignment, i.e., initialize $\boldsymbol{w}_{\mathrm{RF}, l}$ as $\boldsymbol{w}_{\mathrm{RF}, l}=\mathbf{0}_{N_{\mathrm{AC}}}$ and then let $\left[\boldsymbol{w}_{\mathrm{RF}, l}\right]_{\mathcal{I}_{\mathrm{AC}, l}}=\frac{1}{\sqrt{M_{\mathrm{AC}}}}\left[\boldsymbol{a}_{\mathrm{AC}}\left(\widehat{\mu}_{l}^{\mathrm{AC}}, \widehat{\nu}_{l}^{\mathrm{AC}}\right)\right]_{\mathcal{I}_{\mathrm{AC}, l}}$ for $1 \leq l \leq L$. The GTTDU module at aircraft can be also refined to further mitigate the delay-beam squint effects. Since the rough Doppler shift estimates are not precise enough for data transmission, we will use $N_{\text {do }}$ OFDM symbols to estimate the fine Doppler shifts in DL, where how to solve the Doppler squint effect is also considered. To ensure the effective channels within multiple OFDM symbols to be quasi-static observed at the aircraft, the transmitters at BSs still need to perform rough Doppler shift pre-compensation on the transmit signals at this stage.

According to the compensated DL channel matrix $\widetilde{\boldsymbol{H}}_{\mathrm{DL}, l}^{\prime}[\bar{m}]\left[k_{l}\right]$, the received signal $y_{\mathrm{do}, l}^{[\bar{m}]}\left[k_{l}\right]$ at the $k_{l}$ th subcarrier of the $\bar{m}$ th OFDM symbol observed from the $l$ th aircraft RF chain can be expressed as (23) on the bottom of this page. In (23), $k_{l} \in \mathcal{K}_{l}, 1 \leq \bar{m} \leq N_{\mathrm{do}}, \Delta \widetilde{\psi}_{l, k_{l}}=\psi_{l, k_{l}}-\widetilde{\psi}_{l, k_{l}}$ is the residual Doppler shift after compensation with $\widetilde{\psi}_{l, k_{l}}$ being the rough Doppler shift estimates at the $k_{l}$ th subcarrier, and $s_{\mathrm{do}, l}\left[k_{l}\right]=s_{\mathrm{do}, l}^{[\bar{m}]}\left[k_{l}\right]$ for $1 \leq \bar{m} \leq N_{\mathrm{do}}$ and $n_{\mathrm{do}, l}^{[\bar{m}]}\left[k_{l}\right]$ are the transmitted pilot signal and noise, respectively. Since $\Delta \widetilde{\psi}_{l, k_{l}}$ is too small to effectively estimate fine Doppler shifts using the limited OFDM symbols, the compensated phase difference $e^{-\mathrm{j} 2 \pi \widetilde{\psi}_{l, k_{l}}(\bar{m}-1) T_{\text {sym }}}$ of $y_{\mathrm{do}, l}^{[\bar{m}]}\left[k_{l}\right]$ in $(23)$ can be removed to obtain

$$
\begin{aligned}
\bar{y}_{\mathrm{do}, l}^{[\bar{m}]}\left[k_{l}\right] & =\gamma_{\mathrm{do}, l} e^{\mathrm{j}(\bar{m}-1) \nu_{l}^{\psi}} \bar{s}_{\mathrm{do}, l}\left[k_{l}\right] \\
& \times \underbrace{e^{\mathrm{j} \frac{2 \pi f_{s} \underline{v}_{l}}{c}\left(\frac{k_{l}-1}{K}-\frac{1}{2}\right)(\bar{m}-1) T_{\mathrm{sym}}}+\bar{n}_{\mathrm{do}, l}^{[\bar{m}]}\left[k_{l}\right],}_{\widetilde{y}_{\mathrm{do}, l}^{[\bar{m}]}\left[k_{l}\right]\left(\underline{v}_{l}\right)}
\end{aligned}
$$

where $\nu_{l}^{\psi}=2 \pi \psi_{z, l} T_{\text {sym }}$ denotes the virtual Doppler shift, and $\widetilde{y}_{\mathrm{do}, l}^{[\bar{m}]}\left[k_{l}\right]$ and $\bar{n}_{\mathrm{do}, l}^{[\bar{m}]}\left[k_{l}\right]$ are the Doppler squint value and noise, respectively. Considering the signals at $K_{l}$ subcarriers of $N_{\text {do }}$

$$
\begin{aligned}
y_{\mathrm{do}, l}^{[\bar{m}]}\left[k_{l}\right] & =\sqrt{P_{l}} \boldsymbol{w}_{\mathrm{RF}, l}^{\mathrm{H}} \widetilde{\boldsymbol{H}}_{\mathrm{DL}, l}^{\prime[\bar{m}]}\left[k_{l}\right] \boldsymbol{f}_{\mathrm{RF}, l} s_{\mathrm{do}, l}^{[\bar{m}]}\left[k_{l}\right]+n_{\mathrm{do}, l}^{\left[\bar{m}_{l}\right]}\left[k_{l}\right] \\
& =\underbrace{\sqrt{P_{l} G_{l}} \alpha_{l} e^{\mathrm{j} f_{s} \tau_{l}}}_{\gamma_{\mathrm{do}, l}} e^{\mathrm{j} 2 \pi \Delta \widetilde{\psi}_{l, k_{l}}(\bar{m}-1) T_{\mathrm{sym}}} \underbrace{\boldsymbol{w}_{\mathrm{RF}, l}^{\mathrm{H}} \widetilde{\boldsymbol{A}}_{\mathrm{DL}, l}\left[k_{l}\right] \boldsymbol{f}_{\mathrm{RF}, l} e^{\mathrm{j}\left(k_{l}-1\right) \mu_{l}^{\tau}} s_{\mathrm{do}, l}\left[k_{l}\right]}_{\bar{s}_{\mathrm{do}, l}\left[k_{l}\right]}+n_{\mathrm{do}, l}^{[\bar{m}]}\left[k_{l}\right] .
\end{aligned}
$$




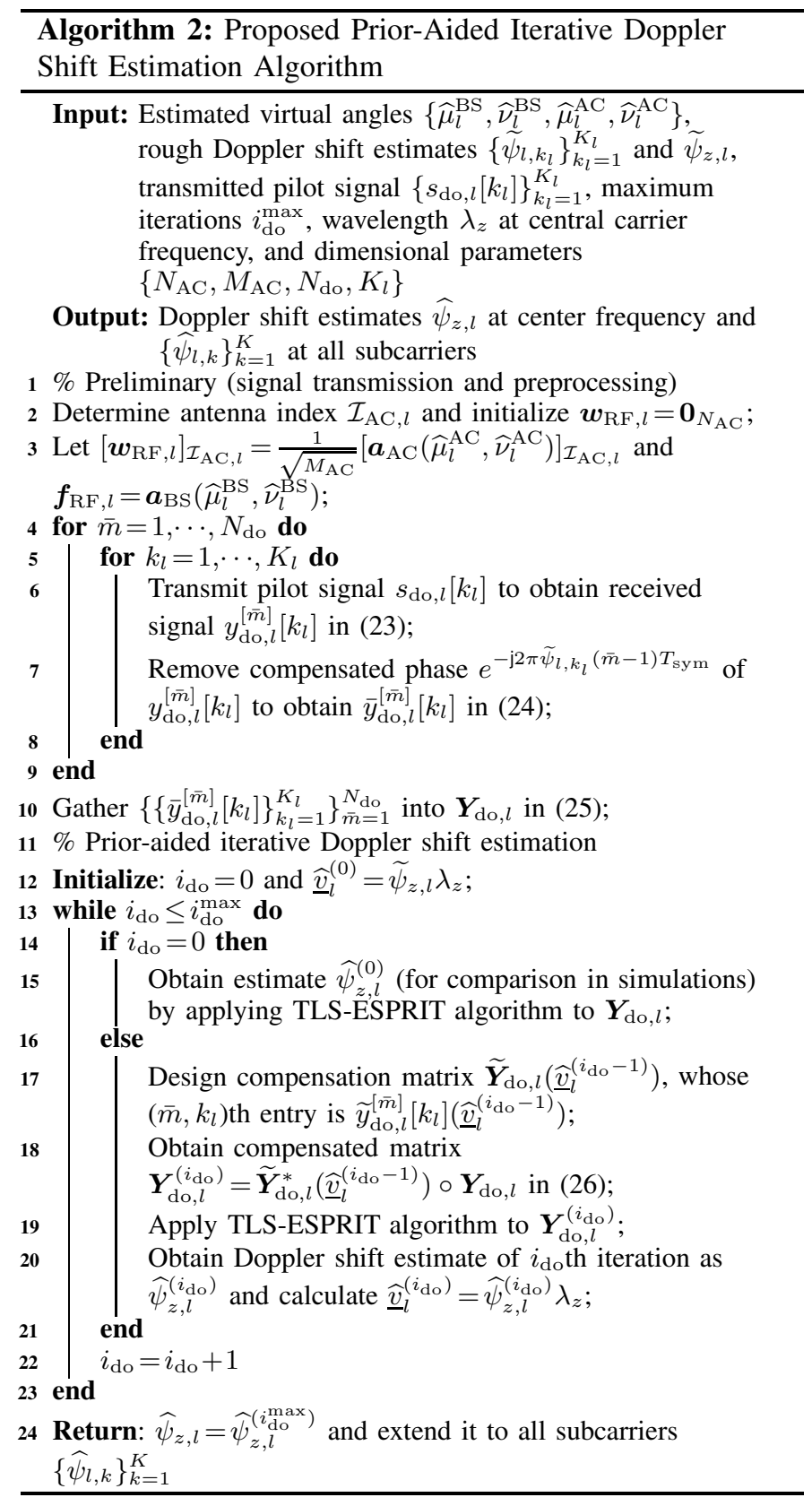

OFDM symbols, we can acquire the received signal matrix $\boldsymbol{Y}_{\mathrm{do}, l} \in \mathbb{C}^{N_{\mathrm{do}} \times K_{l}}$ as

$$
\boldsymbol{Y}_{\mathrm{do}, l}=\gamma_{\mathrm{do}, l}\left(\boldsymbol{a}_{\psi}\left(\nu_{l}^{\psi}, N_{\mathrm{do}}\right) \overline{\boldsymbol{s}}_{\mathrm{do}, l}^{\mathrm{T}}\right) \circ \tilde{\boldsymbol{Y}}_{\mathrm{do}, l}\left(\underline{v}_{l}\right)+\boldsymbol{N}_{\mathrm{do}, l},
$$

where $\boldsymbol{a}_{\psi}\left(\nu_{l}^{\psi}, N_{\mathrm{do}}\right)=\left[1 e^{\mathrm{j} \nu_{l}^{\psi}} \cdots e^{\mathrm{j}\left(N_{\mathrm{do}}-1\right) \nu_{l}^{\psi}}\right]^{\mathrm{T}} \in \mathbb{C}^{N_{\mathrm{do}}}$ denotes the steering vector associated with the Doppler shift $\psi_{z, l}$, $\overline{\boldsymbol{s}}_{\mathrm{do}, l}=\left[\bar{s}_{\mathrm{do}, l}\left[\left\{\mathcal{K}_{l}\right\}_{1}\right] \cdots \bar{s}_{\mathrm{do}, l}\left[\left\{\mathcal{K}_{l}\right\}_{K_{l}}\right]\right]^{\mathrm{T}} \in \mathbb{C}^{K_{l}}, \widetilde{\boldsymbol{Y}}_{\mathrm{do}, l}\left(\underline{v}_{l}\right)$ with $\left[\widetilde{\boldsymbol{Y}}_{\mathrm{do}, l}\left(\underline{v}_{l}\right)\right]_{\bar{m}, k_{l}}=\widetilde{y}_{\mathrm{do}, l}^{[\bar{m}]}\left[k_{l}\right]\left(\underline{v}_{l}\right)$ and $\boldsymbol{N}_{\mathrm{do}, l}$ are the Doppler squint and noise matrices, respectively.

To attenuate the impact of Doppler squint matrix $\widetilde{\boldsymbol{Y}}_{\mathrm{do}, l}\left(\underline{v}_{l}\right)$ on (25), we propose the following prior-aided iterative Doppler shift estimation algorithm. Define the rough Doppler shift estimate at the central carrier frequency as $\widetilde{\psi}_{z, l}$, and the initially relative radial velocity is given by $\underline{\underline{v}}_{l}^{(0)}=\widetilde{\psi}_{z, l} \lambda_{z}$.
At the $i_{\mathrm{do}}$ th iteration, by exploiting the acquired $\widehat{\widehat{v}}_{l}^{\left(i_{\mathrm{do}}-1\right)}$ at the $\left(i_{\mathrm{do}}-1\right)$ th iteration, the compensation matrix can be designed as $\widetilde{\boldsymbol{Y}}_{\mathrm{do}, l}\left(\widehat{\underline{v}}_{l}^{\left(i_{\mathrm{do}}-1\right)}\right)$, and its $\left(\bar{m}, k_{l}\right)$ th element is $\widetilde{y}_{\mathrm{do}, l}^{[\bar{m}]}\left[k_{l}\right]\left(\widehat{\underline{v}}_{l}^{\left(i_{\mathrm{do}}-1\right)}\right)$, which can be acquired by replacing $\underline{v}_{l}$ of $\widetilde{y}_{\mathrm{do}, l}^{[\bar{m}]}\left[k_{l}\right]\left(\underline{v}_{l}\right)$ in (24) with $\underline{\widehat{v}}_{l}^{\left(i_{\mathrm{do}}-1\right)}$. The compensated receive matrix $\boldsymbol{Y}_{\mathrm{do}, l}^{\left(i_{\mathrm{do}}\right)}=\widetilde{\boldsymbol{Y}}_{\mathrm{do}, l}^{*}\left(\underline{\widehat{v}}_{l}^{\left(i_{\mathrm{do}}-1\right)}\right) \circ \boldsymbol{Y}_{\mathrm{do}, l}$ can be then rewritten as

$$
\begin{aligned}
\boldsymbol{Y}_{\mathrm{do}, l}^{\left(i_{\mathrm{do}}\right)}= & \gamma_{\mathrm{do}, l}\left(\boldsymbol{a}_{\psi}\left(\nu_{l}^{\psi}, \bar{m}\right) \overline{\boldsymbol{s}}_{\mathrm{do}, l}^{\mathrm{T}}\right) \\
& \circ\left(\tilde{\boldsymbol{Y}}_{\mathrm{do}, l}\left(\underline{v}_{l}\right) \circ \tilde{\boldsymbol{Y}}_{\mathrm{do}, l}^{*}\left(\widehat{\underline{v}}_{l}^{\left(i_{\mathrm{do}}-1\right)}\right)\right)+\boldsymbol{N}_{\mathrm{do}, l}^{\left(i_{\mathrm{do}}\right)},
\end{aligned}
$$

where $\boldsymbol{N}_{\mathrm{do}, l}^{\left(i_{\mathrm{do}}\right)}$ is the associated noise matrix. According to $\boldsymbol{Y}_{\mathrm{do}, l}^{\left(i_{\mathrm{do}}\right)}$ in (26), we can obtain the Doppler shift estimate at the center frequency of the $i_{\mathrm{do}}$ th iteration, denoted by $\widehat{\psi}_{z, l}^{\left(i_{\text {do }}\right)}$, using Total Least Squares ESPRIT (TLS-ESPRIT) [50]. By employing this estimated $\widehat{\psi}_{z, l}^{\left(i_{\mathrm{do}}\right)}$ to calculate the finely relative radial velocity, i.e., $\widehat{\underline{v}}_{l}^{\left(i_{\mathrm{do}}\right)}=\widehat{\psi}_{z, l}^{\left(i_{\mathrm{do}}\right)} \lambda_{z}$, we can design fine compensation matrix to further improve the accuracy of Doppler estimation. Finally, at the $i_{\mathrm{do}}^{\max }$ th iteration, we can obtain the fine estimates of Doppler shift corresponding to $L$ BSs, i.e., $\widehat{\psi}_{z, l}=\widehat{\psi}_{z, l}^{\left(i_{\mathrm{do}}^{\max }\right)}$, which can be extended to all subcarriers $\left\{\widehat{\psi}_{l, k}\right\}_{k=1}^{K}$ for $1 \leq l \leq L$. The proposed prioraided iterative Doppler shift estimation algorithm above is summarized in Algorithm 2, where the Doppler squint effect can be addressed well.

\section{Path Delay and Channel Gain Estimation}

At the path delay estimation stage, the fine Doppler shift estimates above can be used to accomplish the fine Doppler compensation as shown in Fig. 3 , and $N_{\text {de }}$ OFDM symbols will be utilized to estimate the path delays in DL. Recall that $\boldsymbol{a}_{\tau}\left(\mu_{l}^{\tau}, K_{l}\right)=\left[e^{\mathrm{j}\left(\left\{\mathcal{K}_{l}\right\}_{1}-1\right) \mu_{l}^{\tau}} e^{\mathrm{j}\left(\left\{\mathcal{K}_{l}\right\}_{2}-1\right) \mu_{l}^{\tau}} \cdots e^{\mathrm{j}\left(\left\{\mathcal{K}_{l}\right\}_{K_{l}}-1\right) \mu_{l}^{\tau}}\right]^{\mathrm{T}}$ in (21) denotes the steering vector associated with path delay $\tau_{l}$, and $\mu_{l}^{\tau}=-2 \pi f_{s} \tau_{l} / K$. The DL received signal $y_{\mathrm{de}, l}^{[\bar{n}]}\left[k_{l}\right]$ at the $k_{l}$ th subcarrier of the $\bar{n}$ th OFDM symbol can be expressed as (27) on the top of the next page. In (27), $k_{l} \in \mathcal{K}_{l}, 1 \leq$ $\bar{n} \leq N_{\mathrm{de}}, s_{\mathrm{de}, l}^{[\bar{n}]}=s_{\mathrm{de}, l}^{[\bar{n}]}\left[k_{l}\right]$ for $k_{l} \in \mathcal{K}_{l}$ is the transmitted pilot signal $^{8}, \widetilde{y}_{\mathrm{de}, l}^{[\bar{n}]}\left[k_{l}\right]$ is the error value including the residual beamDoppler squint errors caused by the channel estimation error, and $n_{\mathrm{de}, l}^{[\bar{n}]}\left[k_{l}\right]$ is the noise. By collecting all received signals at $K_{l}$ subcarriers into the vector $\boldsymbol{y}_{\mathrm{de}, l}^{[\bar{n}]} \in \mathbb{C}^{K_{l}}$, we have

$$
\begin{aligned}
\boldsymbol{y}_{\mathrm{de}, l}^{[\bar{n}]} & =\left[y_{\mathrm{de}, l}^{[\bar{n}]}\left[\left\{\mathcal{K}_{l}\right\}_{1}\right] \cdots y_{\mathrm{de}, l}^{[\bar{n}]}\left[\left\{\mathcal{K}_{l}\right\}_{K_{l}}\right]\right]^{\mathrm{T}} \\
& =\gamma_{\mathrm{de}, l} \boldsymbol{a}_{\tau}\left(\mu_{l}^{\tau}, K_{l}\right) \bar{s}_{\mathrm{de}, l}^{[\bar{n}]} \circ \widetilde{\boldsymbol{y}}_{\mathrm{de}, l}^{[\bar{n}]}+\boldsymbol{n}_{\mathrm{de}, l}^{[\bar{n}]},
\end{aligned}
$$

where $\widetilde{\boldsymbol{y}}_{\mathrm{de}, l}^{[\bar{n}]}=\left[\widetilde{y}_{\mathrm{de}, l}^{[\bar{n}]}\left[\left\{\mathcal{K}_{l}\right\}_{1}\right] \cdots \widetilde{y}_{\mathrm{de}, l}^{[\bar{n}]}\left[\left\{\mathcal{K}_{l}\right\}_{K_{l}}\right]\right]^{\mathrm{T}}$ and $\boldsymbol{n}_{\mathrm{de}, l}^{[\bar{n}]}$ denote the error and noise vector, respectively. Considering the received signals of $N_{\text {de }}$ OFDM symbols, we can obtain the matrix $\boldsymbol{Y}_{\mathrm{de}, l}=\left[\boldsymbol{y}_{\mathrm{de}, l}^{[1]} \cdots \boldsymbol{y}_{\mathrm{de}, l}^{\left[N_{\mathrm{de}}\right]}\right] \in \mathbb{C}^{K_{l} \times N_{\mathrm{de}}}$ as

$$
\boldsymbol{Y}_{\mathrm{de}, l}=\gamma_{\mathrm{de}, l}\left(\boldsymbol{a}_{\tau}\left(\mu_{l}^{\tau}, K_{l}\right) \overline{\boldsymbol{s}}_{\mathrm{de}, l}^{\mathrm{T}}\right) \circ \tilde{\boldsymbol{Y}}_{\mathrm{de}, l}+\boldsymbol{N}_{\mathrm{de}, l},
$$

${ }^{8}$ Note that we assume the same pilot signals are adopted by $K_{l}$ subcarriers, which maybe lead to the high PAPR in OFDM systems. Fortunately, we can utilize a predefined pseudo-random descrambling code spread at all subcarriers [37] to reduce the high PAPR effectively. 


$$
\begin{aligned}
& y_{\mathrm{de}, l}^{[\bar{n}]}\left[k_{l}\right]=\sqrt{P_{l}} \boldsymbol{w}_{\mathrm{RF}, l}^{\mathrm{H}} \widetilde{\boldsymbol{H}}_{\mathrm{DL}, l}^{\prime[\bar{n}]}\left[k_{l}\right] \boldsymbol{f}_{\mathrm{RF}, l} s_{\mathrm{de}, l}^{[\bar{n}]}\left[k_{l}\right]+n_{\mathrm{de}, l}^{[\bar{n}]}\left[k_{l}\right] \\
& =\underbrace{\sqrt{P_{l} G_{l}} \alpha_{l} e^{\mathrm{j} \pi f_{s} \tau_{l}} \boldsymbol{w}_{\mathrm{RF}, l}^{\mathrm{H}} \boldsymbol{A}_{\mathrm{DL}, l} \boldsymbol{f}_{\mathrm{RF}, l}}_{\gamma_{\mathrm{de}, l}} e^{\mathrm{j}\left(k_{l}-1\right) \mu_{l}^{\tau}} \underbrace{e^{\mathrm{j} 2 \pi\left(\psi_{z, l}-\widehat{\psi}_{z, l}\right)(\bar{n}-1) T_{\mathrm{sym}}} s_{\mathrm{de}, l}^{[\bar{n}]}}_{\bar{s}_{\mathrm{de}, l}^{(\bar{n})}} \cdot \widetilde{y}_{\mathrm{de}, l}^{[\bar{n}]}\left[k_{l}\right]+n_{\mathrm{de}, l}^{[\bar{n}]}\left[k_{l}\right] .
\end{aligned}
$$

where $\overline{\boldsymbol{s}}_{\mathrm{de}, l}=\left[\bar{s}_{\mathrm{de}, l}^{[1]} \cdots \bar{s}_{\mathrm{de}, l}^{\left[N_{\mathrm{de}}\right]}\right]^{\mathrm{T}} \in \mathbb{C}^{N_{\mathrm{de}}}$, and $\tilde{\boldsymbol{Y}}_{\mathrm{de}, l}=$ $\left[\widetilde{\boldsymbol{y}}_{\mathrm{de}, l}^{[1]} \cdots \widetilde{\boldsymbol{y}}_{\mathrm{de}, l}^{\left[N_{\mathrm{de}}\right]}\right]$ and $\boldsymbol{N}_{\mathrm{de}, l}$ are the residual beam-Doppler squint and noise matrices, respectively. By exploiting the TLSESPRIT algorithm [50], we can obtain the path delay estimates corresponding to $L$ BSs, i.e., $\left\{\widehat{\tau}_{l}\right\}_{l=1}^{L}$. From (29), we observe that the accuracy of path delay estimation depends on the angle and Doppler estimation accuracy, and this conclusion can be further verified by the simulation results in Section VII.

To estimate the channel gains, we need to harness the received signal matrix $\boldsymbol{Y}_{\mathrm{de}, l}$ in (29). Specifically, this matrix $\boldsymbol{Y}_{\mathrm{de}, l}$ can be split into the equivalent channel gain $\bar{\alpha}_{l}$ and $\overline{\boldsymbol{Y}}_{\mathrm{de}, l}$, i.e., $\boldsymbol{Y}_{\mathrm{de}, l}=\bar{\alpha}_{l} \overline{\boldsymbol{Y}}_{\mathrm{de}, l}$, where $\bar{\alpha}_{l}=\sqrt{P_{l} G_{l}} \alpha_{l}$. Regardless of the residual beam-Doppler squint and noise matrices of $\boldsymbol{Y}_{\mathrm{de}, l}$, we can then utilize the previously estimated dominant channel parameters, i.e., the azimuth/elevation angles at BSs and aircraft, Doppler shifts, and path delays, to reestablish the estimated matrix of $\overline{\boldsymbol{Y}}_{\mathrm{de}, l}$ as $\widehat{\overline{\boldsymbol{Y}}}_{\mathrm{de}, l}$. Finally, we can obtain the estimation of $\bar{\alpha}_{l}$, denoted by $\widehat{\alpha}_{l}$, as

$$
\widehat{\alpha}_{l}=\frac{1}{N_{\mathrm{de}} K_{l}} \sum_{\bar{n}=1}^{N_{\mathrm{de}}} \sum_{k_{l}=1}^{K_{l}}\left[\boldsymbol{Y}_{\mathrm{de}, l}\right]_{k_{l}, \bar{n}} /\left[\widehat{\overline{\boldsymbol{Y}}}_{\mathrm{de}, l}\right]_{k_{l}, \bar{n}} .
$$

\section{Data-Aided Channel Tracking}

In Section III, we have acquired the estimates of dominant channel parameters, which will be used for the following data transmission. Although THz UM-MIMO-based aeronautical communication channels exhibit the fast time-varying fading characteristic caused by the large Doppler shifts, the variations of dominant channel parameters, including angles, delays, Doppler shifts, and channel gains, can be relatively smooth within very transitory duration time $T_{\mathrm{sym}}$. Hence, we regard the duration time of $N_{\mathrm{C}}$ OFDM symbols as a Time Interval (TI), and the channel parameters within this TI are assumed to be stationary. Note that after the rough or fine Doppler compensation, the channel related to each OFDM symbol within the same TI is still slowly changing due to the imperfect Doppler compensation. Hence, after a long period of accumulation, the channels can change obviously, which would drastically degrade the detection accuracy of received data. To improve the reliability and efficiency of data transmission, a DADD-based channel tracking algorithm is developed to track the beam-aligned effective channels in real-time, which would save numerous pilot overhead as the time-varying channels should be updated frequently. The proposed DADD-based method utilizes the channel correlation of two adjacent OFDM symbols, where the estimated channels in the previous symbol can be approximately regarded as the real-time channels of the next symbol to detect the data sequentially. Meanwhile, the powerful error correction capability of the channel coding (e.g., Turbo or LDPC codings) can correct part of the erroneous detected data to minimize error propagation during the decision-directed process. Note that at the data transmission stage, we consider $L$ BSs can simultaneously serve the aircraft using the same time-frequency resource to achieve the high spectrum efficiency, i.e., signals associated with different BSs can be distinguished in the spatial domain, rather than the OFDMA utilized for the initial channel estimation. The proposed DADD-based channel tracking algorithm is summarized in Algorithm 3.

Specifically, considering the $r$ th OFDM symbol with $r=$ $(q-1) N_{\mathrm{C}}+p$ that corresponds to the $p$ th OFDM symbol of the $q$ th TI, the DL channel matrix $\boldsymbol{H}_{\mathrm{DL}, l}^{[n]}[k]$ in (4) can be rewritten as $\boldsymbol{H}_{\mathrm{DL}, l}^{[r]}[k]$, which contains the channel parameters $G_{l}^{[q]}, \alpha_{l}^{[q]}$, $\psi_{z, l}^{[q]}, \underline{v}_{l}^{[q]}, \tau_{l}^{[q]}, \theta_{l}^{\mathrm{AC}}[q], \varphi_{l}^{\mathrm{AC}}[q], \theta_{l}^{\mathrm{BS}}[q]$, and $\varphi_{l}^{\mathrm{BS}}[q]$. Define the initial data sequence in the $r$ th OFDM symbol at the $l$ th BS as $\boldsymbol{x}_{l}^{[r]}$, and this sequence can be mapped to $K$ subcarriers via channel coding and modulation to obtain the transmitted signal vector, i.e., $\boldsymbol{s}_{l}^{[r]}=\left[s_{l}^{[r]}[1] \cdots s_{l}^{[r]}[K]\right]^{\mathrm{T}} \in \mathbb{C}^{K}$. The DL baseband signal vector $\boldsymbol{y}^{[r]}[k] \in \mathbb{C}^{L}$ received by aircraft at the $k$ th subcarrier of the $r$ th OFDM symbol can be expressed as

$$
\begin{aligned}
& \boldsymbol{y}^{[r]}[k]=\left[y_{1}^{[r]}[k] \cdots y_{L}^{[r]}[k]\right]^{\mathrm{T}} \\
& =\boldsymbol{W}_{\mathrm{RF}}^{\mathrm{H}}\left(\sum_{l=1}^{L} \sqrt{P_{l}} \widetilde{\boldsymbol{H}}_{\mathrm{DL}, l}^{\prime[r]}[k] \boldsymbol{f}_{\mathrm{RF}, l} s_{l}^{[r]}[k]+\boldsymbol{n}^{[r]}[k]\right),
\end{aligned}
$$

where $1 \leq k \leq K, \boldsymbol{W}_{\mathrm{RF}}=\left[\boldsymbol{w}_{\mathrm{RF}, 1} \cdots \boldsymbol{w}_{\mathrm{RF}, L}\right]$, and $\boldsymbol{n}^{[r]}[k]$ is the noise vector. In (31), the $l$ th received signal $y_{l}^{[r]}[k]$ in $\boldsymbol{y}^{[r]}[k]$ corresponding to the transmitted signal of the $l$ th $\mathrm{BS}$ is given by (32) on the bottom of this page. In (32), the second entry is the interference from other BSs, $n_{l}^{[r]}[k]$ is the combining noise, and $h_{l}^{[r]}[k]$ and $z_{l}^{[r]}[k]$ are the beam-aligned effective channel coefficient and interference plus noise, respectively. Note that the interference entry in (32) is regarded as the additional noise

$$
y_{l}^{[r]}[k]=\underbrace{\sqrt{P_{l}} \boldsymbol{w}_{\mathrm{RF}, l}^{\mathrm{H}} \widetilde{\boldsymbol{H}}_{\mathrm{DL}, l}^{[[r]}[k] \boldsymbol{f}_{\mathrm{RF}, l}}_{h_{l}^{[r]}[k]} s_{l}^{[r]}[k]+\underbrace{\boldsymbol{w}_{\mathrm{RF}, l}^{\mathrm{H}} \sum_{\substack{l^{\prime}=1 \\ l^{\prime} \neq l}}^{L} \sqrt{P_{l^{\prime}}} \widetilde{\boldsymbol{H}}_{\mathrm{DL}, l^{\prime}}^{\prime[r]}[k] \boldsymbol{f}_{\mathrm{RF}, l^{\prime}} s_{l^{\prime}}^{[r]}[k]+n_{l}^{[r]}[k]}_{z_{l}^{[r]}[k]} .
$$




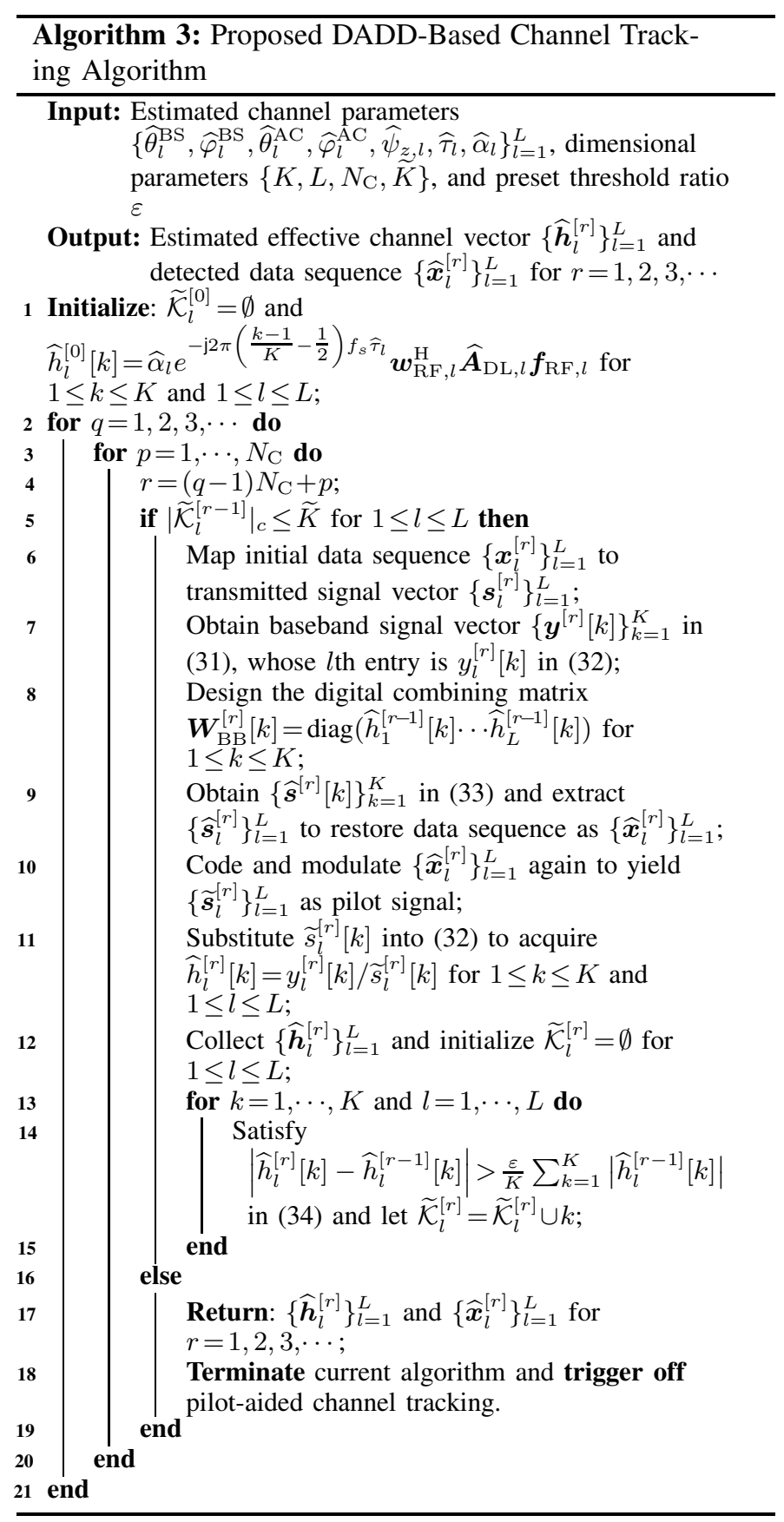

due to the small interference from other BSs caused by the large angle differences among different BSs and the extremely narrow beams formed by $\mathrm{THz}$ UM-MIMO array at aircraft. Thus, (32) can be rewritten as $y_{l}^{[r]}[k]=h_{l}^{[r]}[k] s_{l}^{[r]}[k]+z_{l}^{[r]}[k]$. The $K$ channel coefficients $\left\{h_{l}^{[r]}[k]\right\}_{k=1}^{K}$ can form together the beam-aligned true effective channel vector $\boldsymbol{h}_{l}^{[r]} \in \mathbb{C}^{K}$ at the $k$ th subcarrier in the $r$ th OFDM symbol.

Based on the estimated effective channel coefficient in the $(r-1)$ th OFDM symbol, denoted by $\widehat{h}_{l}^{[r-1]}[k]$ for $1 \leq l \leq L$, we can design the digital combining matrix as $\boldsymbol{W}_{\mathrm{BB}}^{[r]}[k]=$ $\operatorname{diag}\left(\widehat{h}_{1}^{[r-1]}[k] \cdots \widehat{h}_{L}^{[r-1]}[k]\right)$. According to (31), the signal vector $\boldsymbol{s}^{[r]}[k]=\left[s_{1}^{[r]}[k] \cdots s_{L}^{[r]}[k]\right] \in \mathbb{C}^{L}$ can be estimated as

$$
\widehat{\boldsymbol{s}}^{[r]}[k]=\left[\widehat{s}_{1}^{[r]}[k] \cdots \widehat{s}_{L}^{[r]}[k]\right]^{\mathrm{T}}=\left(\boldsymbol{W}_{\mathrm{BB}}^{[r]}[k]\right)^{\mathrm{H}} \boldsymbol{y}^{[r]}[k],
$$

where the $l$ th entry of $\widehat{\boldsymbol{s}}^{[r]}[k]$ is $\widehat{s}_{l}^{[r]}[k]=\frac{\widehat{h}_{l}^{[r]}[k]}{\widehat{h}_{l}^{[r-1]}[k]} s_{l}^{[r]}[k]+\frac{z_{l}^{[r]}[k]}{\widehat{h}_{l}^{[r-1]}[k]}$. By extracting the received signal processed by the $l$ th RF chain and gathering these signals at $K$ subcarriers, the estimation of transmitted signal vector $\boldsymbol{s}_{l}^{[r]}$ can be denoted by $\hat{\boldsymbol{s}}_{l}^{[r]} \in \mathbb{C}^{K}$. To track the effective channel of the current $r$ th OFDM symbol, i.e., $\boldsymbol{h}_{l}^{[r]}$, this signal vector $\widehat{\boldsymbol{s}}_{l}^{[r]}$ can be demodulated and decoded as the detected data sequence $\widehat{\boldsymbol{x}}_{l}^{[r]}$ (i.e., the estimate of initial data sequence $\boldsymbol{x}_{l}^{[r]}$ ). This data sequence $\widehat{x}_{l}^{[r]}$ can be then coded and modulated again to yield the transmitted signal vector $\widetilde{\boldsymbol{s}}_{l}^{[r]}$, which should be more accurate than the estimated $\widehat{\boldsymbol{s}}_{l}^{[r]}$ thanks to the error correction of channel coding. By considering $\widetilde{\boldsymbol{s}}_{l}^{[r]}$ as the pilot signal, we substitute its $k$ th element, denoted by $\widetilde{s}_{l}^{[r]}[k]$, into the received signal $y_{l}^{[r]}[k]$ in (32) to acquire the estimate of effective channel coefficient $h_{l}^{[r]}[k]$, i.e., $\widehat{h}_{l}^{[r]}[k]=y_{l}^{[r]}[k] / \widetilde{s}_{l}^{[r]}[k]$. Finally, considering $K$ subcarriers, the estimated effective channel vector of the $r$ th OFDM symbol is $\widehat{\boldsymbol{h}}_{l}^{[r]} \in \mathbb{C}^{K}$ for $1 \leq l \leq L$. Accordingly, the digital combining matrix at the $k$ th subcarrier in the $(r+1)$ th OFDM symbol can be designed as $\boldsymbol{W}_{\mathrm{BB}}^{[r+1]}[k]=\operatorname{diag}\left(\widehat{h}_{1}^{[r]}[k] \cdots \widehat{h}_{L}^{[r]}[k]\right)$, which is used to perform the subsequent channel equalization. Furthermore, by utilizing the previously estimated channel parameters at the initial channel estimation stage, the estimates of initial beam-aligned effective channel vectors $\left\{\widehat{\boldsymbol{h}}_{l}^{[0]}\right\}_{l=1}^{L}$ can be obtained as $\widehat{h}_{l}^{[0]}[k]=\widehat{\alpha}_{l} e^{-\mathrm{j} 2 \pi\left(\frac{k-1}{K}-\frac{1}{2}\right) f_{s} \widehat{\tau}_{l}} \boldsymbol{w}_{\mathrm{RF}, l}^{\mathrm{H}} \widehat{\boldsymbol{A}}_{\mathrm{DL}, l} \boldsymbol{f}_{\mathrm{RF}, l}$ for $1 \leq k \leq K$ and $1 \leq l \leq L$, where $\widehat{A}_{\mathrm{DL}, l}$ is the reconstructed $\mathrm{DL}$ array response matrix in (5) using the fine angle estimates.

As the time goes on, the previously estimated channel parameters will not match the current effective channels. Therefore, the quality of the tracked effective channel vectors at the data-aided channel tracking stage should be monitored in real-time by exploiting the temporal correlation of two adjacent OFDM symbols. Specifically, for the estimated effective channel vector $\widehat{\boldsymbol{h}}_{l}^{[r]}$ in the $r$ th OFDM symbol, its $k$ th channel coefficient $\widehat{h}_{l}^{[r]}[k]$ can be regarded as a wrong coefficient if $\widehat{h}_{l}^{[r]}[k]$ satisfies

$$
\left|\widehat{h}_{l}^{[r]}[k]-\widehat{h}_{l}^{[r-1]}[k]\right|>\frac{\varepsilon}{K} \sum_{k=1}^{K}\left|\widehat{h}_{l}^{[r-1]}[k]\right|,
$$

where $\varepsilon$ is a preset threshold ratio. The indices of subcarriers involving erroneous coefficients composes a set $\widetilde{\mathcal{K}}_{l}^{[r]}$. Let $\widetilde{K}$ as the acceptable number of erroneous channel coefficients, the tracked effective channel vectors can be regarded as the invalid estimates if $\left|\widetilde{\mathcal{K}}_{l}^{[r]}\right|_{c}>\widetilde{K}$ for $1 \leq l \leq L$, which will trigger off the pilot-aided channel tracking in Section V.

\section{Pilot-Aided Channel Tracking}

In this section, the previously estimated channel parameters in Section III will be exploited as the prior information for facilitating the pilot-aided channel tracking. This is because according to the previous analysis, these channel parameters including angles, Doppler shifts are changing slowly and 


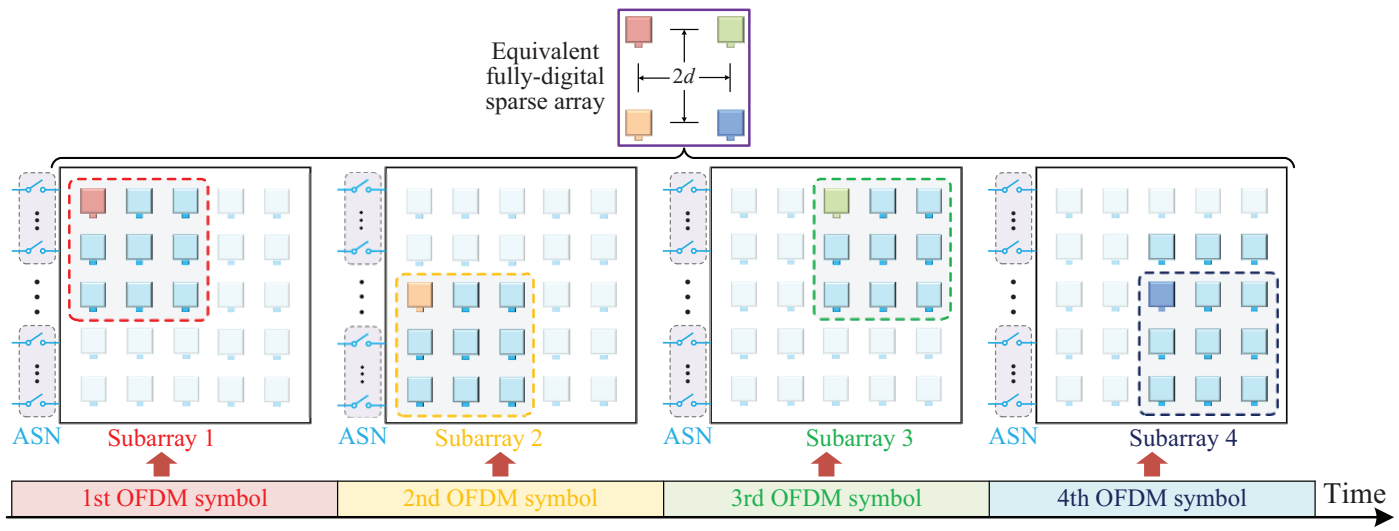

Fig. 8. The schematic diagram of subarray selection based on different antenna connection patterns of the reconfigurable RF selection network at the angle tracking stage. Taking the UPA of size $5 \times 5$ as an example, this UPA can be partitioned into 4 subarrays of size $3 \times 3$, and the interval between each subarray is the width of two antennas. The same RF chain sequentially selects the corresponding subarrays in 4 successive OFDM symbols to receive signals, and these received signals will be equivalent to the signals received by a low-dimensional fully-digital sparse array of size $2 \times 2$ with the sparse spacing $\Omega=2$.

can usually not vary dramatically. Since previously estimated channel parameters can be more accurate than the rough estimates based on navigation information, the tracked channel parameters at this stage would be more accurate than those acquired at the initial channel estimation stage. The main process of the pilot-aided channel tracking is similar to the initial channel estimation in Section III. The difference between them lies in that the azimuth and elevation angles at BSs and aircraft in this section are estimated by forming the array response vector of equivalent low-dimensional fullydigital sparse array. By contrast, an equivalent fully-digital array with critical antenna spacing (i.e., the half-wavelength antenna spacing) is considered in Section III. The existing conclusions indicate that the usage of sparse array can improve the accuracy of angle estimation significantly, but these estimated angles would suffer from the angle ambiguity issue [51], [52]. Fortunately, this angle ambiguity can be solved with the aid of the previously estimated angles. Due to space constraints, this section focuses on the pilot-aided angle tracking at BSs.

Specifically, $I_{\mathrm{BS}}^{\prime}$ OFDM symbols are used to obtain the equivalent fully-digital sparse array of size $I_{\mathrm{BS}}^{\prime \mathrm{h}} \times I_{\mathrm{BS}}^{\prime \mathrm{v}}$ at BSs, where $I_{\mathrm{BS}}^{\prime}=I_{\mathrm{BS}}^{\prime \mathrm{h}} I_{\mathrm{BS}}^{\prime \mathrm{v}}$ subarrays can be acquired by reconfiguring the dedicated connection pattern of the RF selection network. Define $\Omega$ as the sparse antenna spacing relative to the critical antenna spacing $d$. The size of the selected subarray is $\bar{M}_{\mathrm{BS}}^{\prime \mathrm{h}} \times \bar{M}_{\mathrm{BS}}^{\prime \mathrm{v}}$ with $\bar{M}_{\mathrm{BS}}^{\prime}=\bar{M}_{\mathrm{BS}}^{\prime \mathrm{h}} \bar{M}_{\mathrm{BS}}^{\prime \mathrm{v}}$ antenna elements, where $\bar{M}_{\mathrm{BS}}^{\prime \mathrm{h}}=N_{\mathrm{BS}}^{\mathrm{h}}-\Omega\left(I_{\mathrm{BS}}^{\prime \mathrm{h}}-1\right)$ and $\bar{M}_{\mathrm{BS}}^{\prime}=N_{\mathrm{BS}}^{\mathrm{v}}-\Omega\left(I_{\mathrm{BS}}^{\prime} \mathrm{v}-1\right)$. Fig. 8 depicts an example that the UPA with size of $5 \times 5$ can be divided into 4 subarrays of size $3 \times 3$, and these subarrays construct the array response vector of equivalent fully-digital sparse array of size $2 \times 2$ with the sparse spacing $\Omega=2$. Similar to the fine angle estimation at BSs in Section III-A1, we can obtain the homologous UL received signal matrix $\overline{\boldsymbol{Y}}_{\mathrm{UL}, l} \in \mathbb{C}^{I_{\mathrm{BS}}^{\prime} \times K_{l}}$ in (17), where the effective array response vector of the sparse array can be expressed as $\overline{\overline{\boldsymbol{a}}}_{\mathrm{BS}}\left(\bar{\mu}_{l}^{\mathrm{BS}}, \bar{\nu}_{l}^{\mathrm{BS}}\right)=\boldsymbol{a}_{\mathrm{v}}\left(\bar{\nu}_{l}^{\mathrm{BS}}, I_{\mathrm{BS}}^{\prime \mathrm{v}}\right) \otimes \boldsymbol{a}_{\mathrm{h}}\left(\bar{\mu}_{l}^{\mathrm{BS}}, I_{\mathrm{BS}}^{\prime \mathrm{h}}\right) \in \mathbb{C}^{I_{\mathrm{BS}}^{\prime}}$ with $\bar{\mu}_{l}^{\mathrm{BS}}=\Omega \mu_{l}^{\mathrm{BS}}$ and $\bar{\nu}_{l}^{\mathrm{BS}}=\Omega \nu_{l}^{\mathrm{BS}}$. By exploiting the proposed prior-aided iterative angle estimation in Algorithm 1 as before, the estimates of $\bar{\mu}_{l}^{\mathrm{BS}}$ and $\bar{\nu}_{l}^{\mathrm{BS}}$ can be respectively obtained as
$\widehat{\bar{\mu}}_{l}^{\mathrm{BS}}$ and $\widehat{\bar{\nu}}_{l}^{\mathrm{BS}}$ at each iteration. Note that $\widehat{\widehat{\mu}}_{l}^{\mathrm{BS}}$ and $\widehat{\bar{\nu}}_{l}^{\mathrm{BS}}$ suffer from the inherent angle ambiguity problem. To further address this angle ambiguity issue, we define an ordered index set $\mathcal{B}=\left\{-1,-1+\frac{1}{\Omega},-1+\frac{2}{\Omega}, \cdots, 1\right\}$ with $|\mathcal{B}|_{c}=2 \Omega+1$, and let $\widetilde{\bar{\mu}}_{l}^{\mathrm{BS}}=\widehat{\bar{\mu}}_{l}^{\mathrm{BS}} / \Omega$ and $\widetilde{\bar{\nu}}_{l}^{\mathrm{BS}}=\widehat{\bar{\nu}}_{l}^{\mathrm{BS}} / \Omega$. Thus, the estimates of virtual angles corresponding to $\widetilde{\bar{\mu}}_{l}^{\mathrm{BS}}$ and $\widetilde{\bar{\nu}}_{l}^{\mathrm{BS}}$, denoted by $\widehat{\mu}_{l}^{\mathrm{BS}}$ and $\widehat{\nu}_{l}^{\prime} \mathrm{BS}$, should satisfy ${\widehat{\mu}_{l}^{\prime}}^{\mathrm{BS}}=\widetilde{\bar{\mu}}_{l}^{\mathrm{BS}}+b_{\mu}^{\star} \pi$ and ${\widehat{\mu}_{l}^{\prime}}^{\mathrm{BS}}=\widetilde{\bar{\nu}}_{l}^{\mathrm{BS}}+b_{\nu}^{\star} \pi$, where $b_{\mu}^{\star} \in \mathcal{B}$ and $b_{\nu}^{\star} \in \mathcal{B}$ are the optimal indices. Due to the limited elements in $\mathcal{B}$, we adopt the exhaustive method to search for these optimal indices $b_{\mu}^{\star}$ and $b_{\nu}^{\star}$. The previously estimated $\widehat{\mu}_{l}^{\mathrm{BS}}$ and $\widehat{\nu}_{l}^{\mathrm{BS}}$ in Section III-A1 can be regarded as the prior information, i.e., $\widetilde{\mu}_{l}^{\mathrm{BS}}=\widehat{\mu}_{l}^{\mathrm{BS}}$ and $\widetilde{\nu}_{l}^{\mathrm{BS}}=\widehat{\nu}_{l}^{\mathrm{BS}}$, and $b_{\mu}^{\star}$ and $b_{\nu}^{\star}$ can be then obtained as

$$
\begin{aligned}
& b_{\mu}^{\star}=\arg \min _{b_{\mu} \in \mathcal{B}}\left|\widetilde{\bar{\mu}}_{l}^{\mathrm{BS}}+b_{\mu} \pi-\widetilde{\mu}_{l}^{\mathrm{BS}}\right|, \\
& b_{\nu}^{\star}=\arg \min _{b_{\nu} \in \mathcal{B}}\left|\widetilde{\bar{\nu}}_{l}^{\mathrm{BS}}+b_{\nu} \pi-\widetilde{\nu}_{l}^{\mathrm{BS}}\right| .
\end{aligned}
$$

Based on the acquired estimates $\widehat{\mu}_{l}^{\prime} \mathrm{BS}$ and $\widehat{\nu}_{l}^{\prime} \mathrm{BS}$, we can calculate the updated estimates of azimuth and elevation angles at the $l$ th BS as $\widehat{\theta}_{l} \mathrm{BS}$ and $\widehat{\varphi}_{l}^{\prime} \mathrm{BS}$, for $1 \leq l \leq L$. The remaining steps are the same as those in Section III-A1 except that the exhaustive search in (35) and (36) should be taken into account. Finally, we can obtain the fine estimates of azimuth and elevation angles at BSs, denoted by $\left\{\widehat{\theta}_{l}^{\mathrm{BS}}, \widehat{\varphi}_{l}^{\mathrm{BS}}\right\}_{l=1}^{L}$. In a similar way, the fine estimates of azimuth and elevation angles at aircraft can be also acquired as $\left\{\widehat{\theta}_{l}^{\mathrm{AC}}, \widehat{\varphi}_{l}^{\mathrm{AC}}\right\}_{l=1}^{L}$, where $I_{\mathrm{AC}}^{\prime}=I_{\mathrm{AC}}^{\prime \mathrm{h}} I_{\mathrm{AC}}^{\prime} \mathrm{O}$ OFDM symbols are required. Moreover, with the help of the previously estimated Doppler shifts, the updated Doppler shift estimates $\left\{\widehat{\psi}_{z, l}\right\}_{l=1}^{L}$ via the pilot-aided channel tracking will be more accurate than those estimated at the initial channel estimation stage, and so do the estimates of path delays $\left\{\widehat{\tau}_{l}\right\}_{l=1}^{L}$ and channel gains $\left\{\bar{\alpha}_{l}\right\}_{l=1}^{L}$. As shown in Fig. 3, the updated beam-aligned effective channels can be then used for the following data transmission, and the tracked channel parameters will be regarded as the prior information for the next pilot-aided channel tracking.

In order to intuitively describe the relationship among different channel estimation and tracking stages above, the 


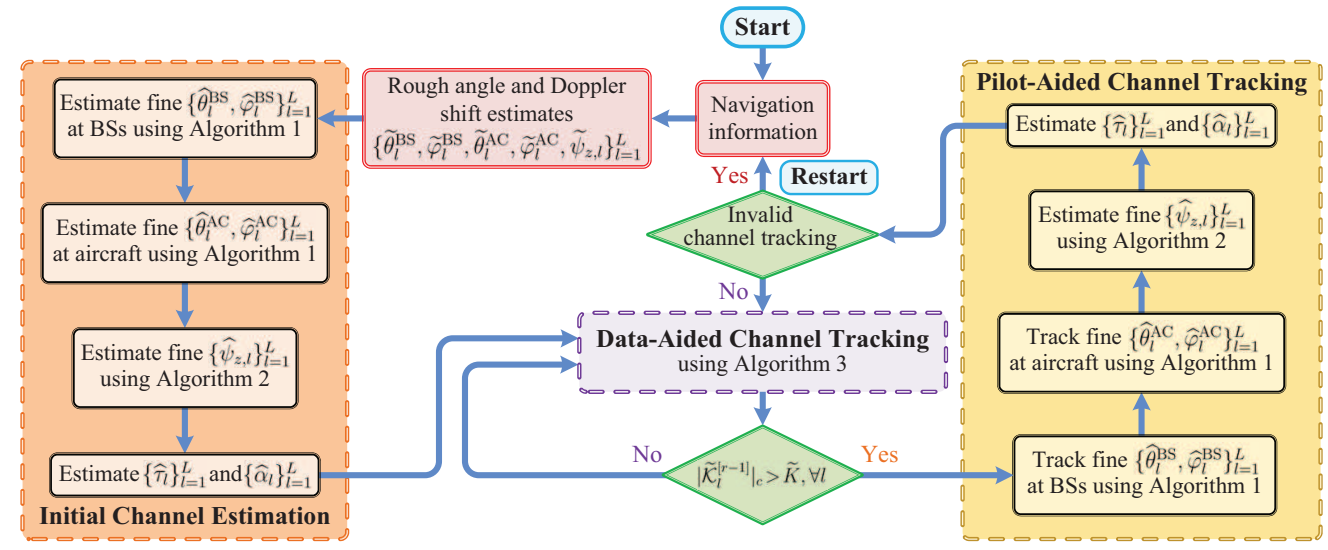

Fig. 9. Flow diagram of the proposed channel estimation and tracking solution.

block diagram of the proposed channel estimation and tracking solution is illustrated in Fig. 9.

\section{PERformance AnAlysis}

\section{A. CRLBs of Channel Parameters}

According to the effective received signal models in Section III, we will investigate the CRLBs of the dominant channel parameters, i.e., azimuth/elevation angles at aerial BSs and aircraft, Doppler shifts, and path delays. Note that practical triple squint effects of aeronautical THz UM-MIMO channels would weaken the accuracy of channel parameter estimation, and these negative effects are not considered in deriving the CRLBs. So these CRLBs serve as the lower-bound of parameter estimation.

1) CRLBs of Angle Estimation at BSs and Aircraft: To investigate the performance at both the initial angle estimation stage and the following angle tracking stage, we consider the received signal model corresponding to the equivalent fullydigital sparse array with size of $\bar{I}_{\mathrm{BS}}^{\mathrm{h}} \times \bar{I}_{\mathrm{BS}}^{\mathrm{v}}$, where the sparse spacing is $\Omega \geq 1$. Based on the expression of (17), the effective received signal model without considering the triple squint effects, denoted by $\overline{\boldsymbol{Y}}_{\mathrm{UL}, l}=\left[\overline{\boldsymbol{y}}_{\mathrm{UL}, l}\left[\left\{\mathcal{K}_{l}\right\}_{1}\right] \cdots \overline{\boldsymbol{y}}_{\mathrm{UL}, l}\left[\left\{\mathcal{K}_{l}\right\}_{K_{l}}\right]\right] \in$ $\mathbb{C}^{\bar{I}_{\mathrm{BS}} \times K_{l}}$, can be written as

$$
\overline{\boldsymbol{Y}}_{\mathrm{UL}, l}=\gamma_{\mathrm{UL}, l} \overline{\overline{\boldsymbol{a}}}_{\mathrm{BS}}\left(\bar{\mu}_{l}^{\mathrm{BS}}, \bar{\nu}_{l}^{\mathrm{BS}}\right) \boldsymbol{s}_{\mathrm{UL}, l}^{\mathrm{T}}+\overline{\boldsymbol{N}}_{\mathrm{UL}, l},
$$

where $1 \leq l \leq L, \bar{I}_{\mathrm{BS}}=\bar{I}_{\mathrm{BS}}^{\mathrm{h}} \bar{I}_{\mathrm{BS}}^{\mathrm{v}}, \quad \overline{\overline{\boldsymbol{a}}}_{\mathrm{BS}}\left(\bar{\mu}_{l}^{\mathrm{BS}}, \bar{\nu}_{l}^{\mathrm{BS}}\right)=$ $\boldsymbol{a}_{\mathrm{v}}\left(\bar{\nu}_{l}^{\mathrm{BS}}, \bar{I}_{\mathrm{BS}}^{\mathrm{v}}\right) \otimes \boldsymbol{a}_{\mathrm{h}}\left(\bar{\mu}_{l}^{\mathrm{BS}}, \bar{I}_{\mathrm{BS}}^{\mathrm{h}}\right) \in \mathbb{C}^{I_{\mathrm{BS}}}$ with $\bar{\mu}_{l}^{\mathrm{BS}}=\Omega \mu_{l}^{\mathrm{BS}}=$ $\Omega \pi \sin \left(\theta_{l}^{\mathrm{BS}}\right) \cos \left(\varphi_{l}^{\mathrm{BS}}\right)$ and $\bar{\nu}_{l}^{\mathrm{BS}}=\Omega \nu_{l}^{\mathrm{BS}}=\Omega \pi \sin \left(\varphi_{l}^{\mathrm{BS}}\right)$, and $\overline{\mathbf{N}}_{\mathrm{UL}, l}$ is the noise matrix with its entry following $\mathcal{C} \mathcal{N}\left(0, \sigma_{n}^{2}\right)$. The likelihood function of $\overline{\boldsymbol{Y}}_{\mathrm{UL}, l}$ is $p\left(\overline{\boldsymbol{Y}}_{\mathrm{UL}, l} ; \boldsymbol{\eta}_{l}\right)$, and the corresponding the log-likelihood function can be expressed as (38) on the bottom of this page by defining $\boldsymbol{\eta}_{l}=\left[\alpha_{l},\left(\boldsymbol{\xi}_{l}^{\mathrm{BS}}\right)^{\mathrm{T}}\right]^{\mathrm{T}}$ with $\boldsymbol{\xi}_{l}^{\mathrm{BS}}=\left[\bar{\nu}_{l}^{\mathrm{BS}}, \bar{\mu}_{l}^{\mathrm{BS}}\right]^{\mathrm{T}}$. Thus, the $(i, j)$ th entry of Fisher Information Matrix (FIM), denoted by $\left[\boldsymbol{G}\left(\boldsymbol{\eta}_{l}\right)\right]_{i, j}$, is given by

$$
\left[\boldsymbol{G}\left(\boldsymbol{\eta}_{l}\right)\right]_{i, j}=-\mathbb{E}\left(\frac{\partial^{2} \ln p\left(\overline{\boldsymbol{Y}}_{\mathrm{UL}, l} ; \boldsymbol{\eta}_{l}\right)}{\partial\left[\boldsymbol{\eta}_{l}\right]_{i} \partial\left[\boldsymbol{\eta}_{l}\right]_{j}}\right) .
$$

According to the results in [53], [54], the CRLB of $\xi_{l}^{\mathrm{BS}}$ consisting of the virtual angles $\bar{\mu}_{l}^{\mathrm{BS}}$ and $\bar{\nu}_{l}^{\mathrm{BS}}$ can be expressed as (40) on the bottom of this page. In (40), $\boldsymbol{B}_{\mathrm{BS}, k_{l}}=\boldsymbol{I}_{2} \otimes s_{\mathrm{UL}, l}\left[\left\{\mathcal{K}_{l}\right\}_{k_{l}}\right], \quad \boldsymbol{\Gamma}_{\mathrm{BS}}=$ $\left[\boldsymbol{a}_{\mathrm{v}}\left(\bar{\nu}_{l}^{\mathrm{BS}}, \bar{I}_{\mathrm{BS}}^{\mathrm{v}}\right) \otimes \frac{\partial \boldsymbol{a}_{\mathrm{h}}\left(\bar{\mu}_{l}^{\mathrm{BS}}, \bar{I}_{\mathrm{BS}}^{\mathrm{h}}\right)}{\partial \bar{\mu}_{l}^{\mathrm{BS}}}, \frac{\partial \boldsymbol{a}_{\mathrm{v}}\left(\bar{\nu}_{l}^{\mathrm{BS}}, \bar{I}_{\mathrm{BS}}^{\mathrm{v}}\right)}{\partial \bar{\nu}_{l}^{\mathrm{BS}}} \otimes \boldsymbol{a}_{\mathrm{h}}\left(\bar{\mu}_{l}^{\mathrm{BS}}, \bar{I}_{\mathrm{BS}}^{\mathrm{h}}\right)\right]$, and the projection operator $\boldsymbol{\Phi}_{\mathrm{BS}}=\overline{\overline{\boldsymbol{a}}}_{\mathrm{BS}}\left(\bar{\mu}_{l}^{\mathrm{BS}}, \bar{\nu}_{l}^{\mathrm{BS}}\right)$ $\times\left(\overline{\overline{\boldsymbol{a}}}_{\mathrm{BS}}^{\mathrm{H}}\left(\bar{\mu}_{l}^{\mathrm{BS}}, \bar{\nu}_{l}^{\mathrm{BS}}\right) \overline{\overline{\boldsymbol{a}}}_{\mathrm{BS}}\left(\bar{\mu}_{l}^{\mathrm{BS}}, \bar{\nu}_{l}^{\mathrm{BS}}\right)\right)^{-1} \overline{\overline{\boldsymbol{a}}}_{\mathrm{BS}}^{\mathrm{H}}\left(\bar{\mu}_{l}^{\mathrm{BS}}, \bar{\nu}_{l}^{\mathrm{BS}}\right)$.

To obtain the CRLBs of azimuth and elevation angles, we define the transformation relationship between the virtual angles and the corresponding physical angles as

$$
\boldsymbol{J}\left(\boldsymbol{\xi}_{l}^{\mathrm{BS}}\right)=\left[\begin{array}{c}
\varphi_{l}^{\mathrm{BS}} \\
\theta_{l}^{\mathrm{BS}}
\end{array}\right]=\left[\begin{array}{c}
\arcsin \left(\frac{\bar{\nu}_{l}^{\mathrm{BS}}}{\Omega \pi}\right) \\
\arcsin \left(\frac{\bar{\mu}_{l}^{\mathrm{BS}}}{\Omega \pi \cos \left(\varphi_{l}^{\mathrm{BS}}\right)}\right)
\end{array}\right] .
$$

Based on the transformation of vector parameter CRLB in [55], defining $\partial \boldsymbol{J}\left(\boldsymbol{\xi}_{l}^{\mathrm{BS}}\right) / \partial \boldsymbol{\xi}_{l}^{\mathrm{BS}}$ as the Jacobian matrix, the CRLBs of azimuth angle $\theta_{l}^{\mathrm{BS}}$ and elevation angle $\varphi_{l}^{\mathrm{BS}}$, denoted by $\operatorname{CRLB}_{\theta_{l}^{\mathrm{BS}}}(\Omega)$ and $\operatorname{CRLB}_{\varphi_{l}^{\mathrm{BS}}}(\Omega)$, can be then formulated as (42) and (43), respectively, on the top of the next page. Finally, the CRLBs of angles at BSs can be obtained as $\operatorname{CRLB}_{\theta^{\mathrm{BS}}}(\Omega)=\frac{1}{L} \sum_{l=1}^{L} \operatorname{CRLB}_{\theta_{l}^{\mathrm{BS}}}(\Omega)$ and $\operatorname{CRLB}_{\varphi^{\mathrm{BS}}}(\Omega)=$ $\frac{1}{L} \sum_{l=1}^{L} \operatorname{CRLB}_{\varphi_{l}^{\mathrm{BS}}}(\Omega)$, respectively. Furthermore, the CRLBs of angles at aircraft, i.e., $\operatorname{CRLB}_{\theta^{\mathrm{AC}}}(\Omega)$ and $\operatorname{CRLB}_{\varphi^{\mathrm{AC}}}(\Omega)$,

$$
\begin{aligned}
\ln p\left(\overline{\boldsymbol{Y}}_{\mathrm{UL}, l} ; \boldsymbol{\eta}_{l}\right)= & -\bar{I}_{\mathrm{BS}} K_{l} \ln \left(\pi \sigma_{n}^{2}\right)-\frac{1}{\sigma_{n}^{2}} \sum_{k_{l}=1}^{K_{l}}\left(\left[\overline{\boldsymbol{y}}_{\mathrm{UL}, l}\left[\left\{\mathcal{K}_{l}\right\}_{k_{l}}\right]-\gamma_{\mathrm{UL}, l} \overline{\overline{\boldsymbol{a}}}_{\mathrm{BS}}\left(\bar{\mu}_{l}^{\mathrm{BS}}, \bar{\nu}_{l}^{\mathrm{BS}}\right) s_{\mathrm{UL}, l}\left[\left\{\mathcal{K}_{l}\right\}_{k_{l}}\right]\right]^{\mathrm{H}}\right. \\
& \left.\times\left[\overline{\boldsymbol{y}}_{\mathrm{UL}, l}\left[\left\{\mathcal{K}_{l}\right\}_{k_{l}}\right]-\gamma_{\mathrm{UL}, l} \overline{\overline{\boldsymbol{a}}}_{\mathrm{BS}}\left(\bar{\mu}_{l}^{\mathrm{BS}}, \bar{\nu}_{l}^{\mathrm{BS}}\right) s_{\mathrm{UL}, l}\left[\left\{\mathcal{K}_{l}\right\}_{k_{l}}\right]\right]\right) . \\
\mathrm{CRLB}_{\boldsymbol{\xi}_{l}^{\mathrm{BS}}}= & \boldsymbol{G}^{-1}\left(\boldsymbol{\eta}_{l}\right)=\frac{\sigma_{n}^{2}}{2\left|\gamma_{\mathrm{UL}, l}\right|^{2}}\left\{\sum_{k_{l}=1}^{K_{l}} \Re\left\{\boldsymbol{B}_{\mathrm{BS}, k_{l}}^{\mathrm{H}} \boldsymbol{\Gamma}_{\mathrm{BS}}^{\mathrm{H}}\left(\boldsymbol{I}_{\bar{I}_{\mathrm{BS}}}-\boldsymbol{\Phi}_{\mathrm{BS}}\right) \boldsymbol{\Gamma}_{\mathrm{BS}} \boldsymbol{B}_{\mathrm{BS}, k_{l}}\right\}\right\}^{-1} .
\end{aligned}
$$




$$
\begin{aligned}
\operatorname{CRLB}_{\varphi_{l}^{\mathrm{BS}}}(\Omega) & =\left[\frac{\partial \boldsymbol{J}\left(\boldsymbol{\xi}_{l}^{\mathrm{BS}}\right)}{\partial \boldsymbol{\xi}_{l}^{\mathrm{BS}}} \operatorname{CRLB}_{\boldsymbol{\xi}_{l}^{\mathrm{BS}}} \frac{\partial \boldsymbol{J}\left(\boldsymbol{\xi}_{l}^{\mathrm{BS}}\right)^{\mathrm{T}}}{\partial \boldsymbol{\xi}_{l}^{\mathrm{BS}}}\right]_{1,1}=\frac{\left[\mathrm{CRLB}_{\boldsymbol{\xi}_{l}^{\mathrm{BS}}}\right]_{1,1}}{\Omega^{2}\left(\pi^{2}-\left(\nu_{l}^{\mathrm{BS}}\right)^{2}\right)}, \\
\mathrm{CRLB}_{\theta_{l}^{\mathrm{BS}}}(\Omega) & =\left[\frac{\partial \boldsymbol{J}\left(\boldsymbol{\xi}_{l}^{\mathrm{BS}}\right)}{\partial \boldsymbol{\xi}_{l}^{\mathrm{BS}}} \operatorname{CRLB}_{\boldsymbol{\xi}_{l}^{\mathrm{BS}}} \frac{\partial \boldsymbol{J}\left(\boldsymbol{\xi}_{l}^{\mathrm{BS}}\right)^{\mathrm{T}}}{\partial \boldsymbol{\xi}_{l}^{\mathrm{BS}}}\right]_{2,2}=\frac{\left[\mathrm{CRLB}_{\boldsymbol{\xi}_{l}^{\mathrm{BS}}}\right]_{2,2}}{\Omega^{2}\left(\pi^{2} \cos ^{2}\left(\varphi_{l}^{\mathrm{BS}}\right)-\left(\mu_{l}^{\mathrm{BS}}\right)^{2}\right)} . \\
\mathrm{CRLB}_{\nu_{l}^{\psi}} & =\frac{\sigma_{n}^{2}}{2\left|\gamma_{\mathrm{do}, l}\right|^{2}}\left\{\sum_{k_{l}=1}^{K_{l}} \Re\left\{\left|\bar{s}_{\mathrm{do}, l}\left[\left\{\mathcal{K}_{l}\right\}_{k_{l}}\right]\right|^{2}\left(\frac{\partial \boldsymbol{a}_{\psi}\left(\nu_{l}^{\psi}, N_{\mathrm{do}}\right)}{\partial \nu_{l}^{\psi}}\right)^{\mathrm{H}}\left(\boldsymbol{I}_{N_{\mathrm{do}}}-\boldsymbol{\Phi}_{\mathrm{Do}}\right) \frac{\partial \boldsymbol{a}_{\psi}\left(\nu_{l}^{\psi}, N_{\mathrm{do}}\right)}{\partial \nu_{l}^{\psi}}\right\}\right\}^{-1}, \\
\mathrm{CRLB}_{\mu_{l}^{\tau}} & =\frac{\sigma_{n}^{2}}{2\left|\gamma_{\mathrm{de}, l}\right|^{2}}\left\{\sum_{n=1}^{N_{\mathrm{de}}} \Re\left\{\left|\bar{s}_{\mathrm{de}, l}^{[n]}\right|^{2}\left(\frac{\partial \boldsymbol{a}_{\tau}\left(\mu_{l}^{\tau}, K_{l}\right)}{\partial \mu_{l}^{\tau}}\right)^{\mathrm{H}}\left(\boldsymbol{I}_{N_{\mathrm{de}}}-\boldsymbol{\Phi}_{\mathrm{De}}\right) \frac{\partial \boldsymbol{a}_{\tau}\left(\mu_{l}^{\tau}, K_{l}\right)}{\partial \mu_{l}^{\tau}}\right\}\right\}^{-1} .
\end{aligned}
$$

can be also acquired in a similar way, where the detailed derivations are omitted due to space constraints.

Remark 2: According to (40), if the system configuration parameters of the transceiver are the same except for different sparse spacing $\Omega$, the CRLB of $\boldsymbol{\xi}_{l}^{\mathrm{BS}}$, i.e., $\mathrm{CRLB}_{\boldsymbol{\xi}_{l}^{\mathrm{BS}}}$, is a constant. Therefore, we can observe from (42) and (43) that $\operatorname{CRLB}_{\theta^{\mathrm{BS}}}(1)$ and $\mathrm{CRLB}_{\varphi_{1}^{\mathrm{BS}}}(1)$ for $\Omega=1$ are the $\Omega^{2}$ times as much as $\operatorname{CRLB}_{\theta_{l}^{\mathrm{BS}}}(\Omega)$ and $\operatorname{CRLB}_{\varphi_{l}^{\mathrm{BS}}}(\Omega)$ for $\Omega>1$, respectively. In other words, compared with the array with critical antenna spacing, the CRLB of sparse array with sparse spacing $\Omega>1$ can achieve the about $20 \lg \Omega \mathrm{dB}$ Mean Square Error (MSE) performance gain, which theoretically testifies the improved accuracy of angle estimation using sparse array.

2) CRLBs of Doppler Shift and Path Delay Estimation: Similar to the CRLB derivations of angle estimation, according to (25) and (29), the CRLBs of virtual Doppler $\nu_{l}^{\psi}$ and virtual delay $\mu_{l}^{\tau}$ can be obtained directly as (44) and (45), respectively, on the top of this page. In (44) and (45), the projection operators $\boldsymbol{\Phi}_{\mathrm{Do}}$ and $\boldsymbol{\Phi}_{\mathrm{De}}$ have the similar form to $\boldsymbol{\Phi}_{\mathrm{BS}}$. By exploiting the transformation of parameter CRLB [55], the CRLBs of Doppler shift $\psi_{z, l}$ and the normalized delay $\bar{\tau}_{l}=f_{s} \tau_{l}$ can be then expressed as $\operatorname{CRLB}_{\psi_{z, l}}=\frac{\mathrm{CRLB}_{\nu} \psi}{\left(2 \pi T_{\mathrm{sym}}\right)^{2}}$ and $\mathrm{CRLB}_{\bar{\tau}_{l}}=\frac{K^{2} \mathrm{CRLB}_{\mu_{l}^{\tau}}}{(2 \pi)^{2}}$, respectively. Finally, the CRLBs of Doppler shift and the normalized delay for $L$ BSs can be acquired as $\mathrm{CRLB}_{\psi_{z}}=\frac{1}{L} \sum_{l=1}^{L} \mathrm{CRLB}_{\psi_{z, l}}$ and $\mathrm{CRLB}_{\bar{\tau}}=$ $\frac{1}{L} \sum_{l=1}^{L} \operatorname{CRLB}_{\bar{\tau}_{l}}$, respectively.

\section{B. Computational Complexity}

The computational complexity of the proposed channel estimation and tracking scheme mainly consists of two portions. The first one is to estimate and track the channel parameters, including the acquisition of azimuth/elevation angles at BSs and aircraft, Doppler shifts, and path delays using TDU-ESPRIT and TLS-ESPRIT algorithms. Since a mass of trivial computations with small computational complexity can be ignored, we focus on the dominant calculation steps involving numerous complex multiplications. For the estimation and tracking of angles at BSs and aircraft, their total computational complexity is $\mathrm{O}\left(2 L I_{\mathrm{BS}} K_{l}+2 L I_{\mathrm{AC}} K_{l}+2 L I_{\mathrm{BS}}^{\prime} K_{l}+2 L I_{\mathrm{AC}}^{\prime} K_{l}\right)$, where $\mathrm{O}(N)$ stands for "on the order of $N$ ". The computational complexity of Doppler shift and path delay estimation is
$\mathrm{O}\left(8 L N_{\mathrm{Do}}^{2} K_{l}+8 L K_{l}^{2} N_{\mathrm{De}}\right)$. The second part is the data-aided channel tracking, and its computational complexity consists of the reestablishment of initial beam-aligned effective channel vectors and the tracking of subsequent effective channel vectors, i.e., $\mathrm{O}\left(L\left(N_{\mathrm{AC}}+N_{\mathrm{BS}}+3 K\right)\right)$ and $\mathrm{O}(L K)$, respectively. It can be seen from the above analysis that although the $\mathrm{THz}$ UM-MIMO arrays employing tens of thousands of antennas are equipped at BSs and aircraft, the computational complexity of the proposed solution is in polynomial time, since the effective low-dimensional signals at the receiver are utilized to estimate and track the aeronautical THz UM-MIMO channels. The state-of-the-art Digital Signal Processing (DSP) hardware devices, such as the latest Field Programmable Gate Array (FPGA), are capable of the operations with the order of trillions of Floating-Point Operations Per Second (FLOPS), which can be used for the proposed solution in $\mathrm{THz}$ UMMIMO-based aeronautical communications with the acceptable processing time.

\section{NUMERICAL EVALUATION}

\section{A. Simulation Setup}

In this section, we evaluate the performance of the proposed channel estimation and tracking scheme for THz UM-MIMObased aeronautical communications, where the simulation scenario considered can be shown in Fig. 10. Without loss of generality, we set the reference altitudes of $L=2$ suspended aerial BSs and an aircraft in Fig. 10(a) are 20 kilometer $(\mathrm{km})$ and $D_{\mathrm{AC}}=10 \mathrm{~km}$ (at the top of the troposphere or the bottom of the stratosphere), respectively, and thus, the vertical distance between the aircraft and BSs is $D_{\mathrm{AB}}=10 \mathrm{~km}$. The distance between two BSs is $D_{\mathrm{BS}}=200 \mathrm{~km}$. In addition, we can abstract a spatial coordinate system as Fig. 10(b) from this real scenario, where point $O$ is the origin of coordinates, and the coordinates of points $A, B$, and $C$ are $\left(0,0, D_{\mathrm{AB}}\right),\left(0, D_{\mathrm{BS}}, D_{\mathrm{AB}}\right)$, and $\left(D_{\mathrm{BS}} / 2, D_{\mathrm{BS}} / 2,0\right)$, respectively. The position coordinate of the aircraft randomly appears in a horizontal circular plane with $C$ as the center and $R_{\mathrm{a}}=50 \mathrm{~km}$ as the radius, and the horizontal direction of aircraft $\boldsymbol{v}_{d}$ with flight speed $v_{\mathrm{AC}}=200$ meter per second $(\mathrm{m} / \mathrm{s})$ falls in the intersection angle OCD. In order to simplify the simulation scenario, we consider that the altitude changes of aerial BSs and aircraft are reflected in the angle change over time. 


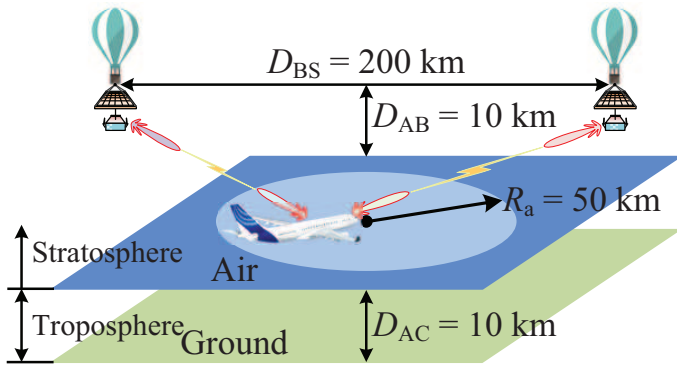

(a)

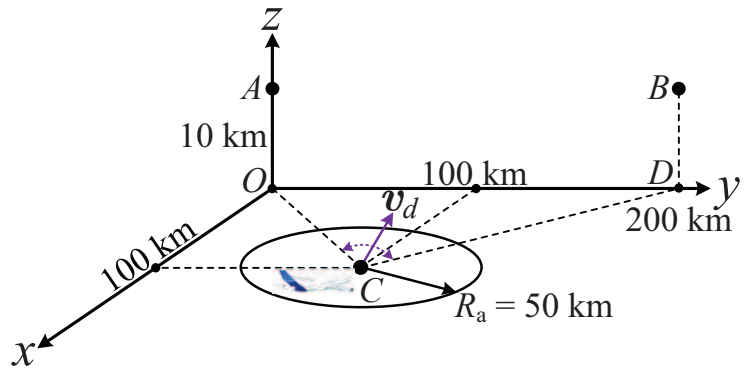

(b)

Fig. 10. (a) Schematic diagram of simulation scenario, and (b) the corresponding spatial coordinate representation.

In simulations, the central carrier frequency is $f_{z}=0.1 \mathrm{THz}$ with system bandwidth $f_{s}=1 \mathrm{GHz}$, the horizontal/vertical antenna numbers of all subarrays at BSs and aircraft are $N_{\mathrm{BS}}^{\mathrm{h}}=N_{\mathrm{BS}}^{\mathrm{v}}=M_{\mathrm{AC}}^{\mathrm{h}}=M_{\mathrm{AC}}^{\mathrm{v}}=200$, and the horizontal and vertical numbers of subarrays at aircraft are $\widetilde{I}_{\mathrm{AC}}^{\mathrm{h}}=1$ and $\widetilde{I}_{\mathrm{AC}}^{\mathrm{v}}=2$, respectively, while the dimensions of the selected equivalent fully-digital (sparse) array are $I_{\mathrm{BS}}^{\mathrm{h}}=I_{\mathrm{BS}}^{\mathrm{v}}=I_{\mathrm{AC}}^{\mathrm{h}}=$ $I_{\mathrm{AC}}^{\mathrm{v}}=5\left(I_{\mathrm{BS}}^{\prime \mathrm{h}}=I_{\mathrm{BS}}^{\prime}=I_{\mathrm{AC}}^{\prime \mathrm{h}}=I_{\mathrm{AC}}^{\prime \mathrm{v}}=5\right)$. The numbers of antennas in each antenna group used for the GTTDU modules at BSs and aircraft are $\widetilde{M}_{\mathrm{BS}}^{\mathrm{h}}=\widetilde{M}_{\mathrm{BS}}^{\mathrm{v}}=\widetilde{M}_{\mathrm{AC}}^{\mathrm{h}}=\widetilde{M}_{\mathrm{AC}}^{\mathrm{v}}=5$. Moreover, the number of OFDM symbols used to estimate and track the Doppler shifts and path delays are $N_{\text {do }}=6$ and $N_{\mathrm{de}}=10$, respectively. The number of subcarriers is set to $K=2048$ with the length of Cyclic Prefix (CP) being $N_{\mathrm{cp}}=128$, and perfect frame synchronization and reliable delay compensation are assumed. The channel parameters are listed as follows. The azimuth and elevation angles at BSs and aircraft $\left\{\theta_{l}^{\mathrm{BS}}, \varphi_{l}^{\mathrm{BS}}, \theta_{l}^{\mathrm{AC}}, \varphi_{l}^{\mathrm{AC}}\right\}_{l=1}^{L}$ are generated from $[-\pi / 3, \pi / 3]$ randomly. Note that due to the long distance between the adjacent BSs, $\left\{\theta_{l}^{\mathrm{AC}}, \varphi_{l}^{\mathrm{AC}}\right\}_{l=1}^{L}$ corresponding to different BSs have the large gaps, and these angles can be set based on the position of aircraft in Fig. 10(b). The Doppler shifts $\left\{\psi_{z, l}\right\}_{l=1}^{L}$ can be set based on $\boldsymbol{v}_{d}$ and the relationship between spatial coordinates of the BSs and aircraft. The path delay $\tau_{l}$ follows uniform distribution $\mathcal{U}\left[0, N_{\mathrm{cp}} T_{s}\right]$ and each of channel gains $\alpha_{l}$ is generated according to $\mathcal{C N}(0,1)$, i.e., $\sigma_{\alpha}^{2}=1$, for $1 \leq l \leq L$. The rough estimates of azimuth/elevation angles at BSs and aircraft $\left\{\widetilde{\theta}_{l}^{\mathrm{BS}}, \widetilde{\varphi}_{l}^{\mathrm{BS}}, \widetilde{\theta}_{l}^{\mathrm{AC}}, \widetilde{\varphi}_{l}^{\mathrm{AC}}\right\}_{l=1}^{L}$ can be randomly selected from the range of these true angles with offset $\pm 5^{\circ}$, while the rough Doppler shift estimate $\widetilde{\psi}_{z, l}$ can be randomly selected from the range of the true $\psi_{z, l}$ with offset $\pm 0.01 \psi_{z, l}$ for $1 \leq l \leq L$. Furthermore, to describe the fast time-varying fading channels, we define the relationship of these channel parameters between the $q$ th and $(q+1)$ th TIs as $x^{[q+1]}=x^{[q]}+s_{\mathrm{pm}} \rho_{x} N_{\mathrm{C}} T_{\mathrm{sym}}$, where $x$ represents the channel parameter coming from $\alpha_{l}, \tau_{l}, \psi_{z, l}, \theta_{l}^{\mathrm{AC}}, \varphi_{l}^{\mathrm{AC}}$, $\theta_{l}^{\mathrm{BS}}$, or $\varphi_{l}^{\mathrm{BS}}$. Here, $s_{\mathrm{pm}}$ denotes a binary variable selected from 1 or -1 randomly, $N_{\mathrm{C}}=70, T_{\mathrm{sym}}=\left(N_{\mathrm{cp}}+K\right) T_{s}=$ 2.176 Microseconds $(\mu \mathrm{s})$, and the duration time of one TI is $T_{\mathrm{TI}}=N_{\mathrm{C}} T_{\mathrm{sym}}=152.32 \mu \mathrm{s}$, while $\rho_{x}$ is the rate of change associated with $x$. We consider $\rho_{\alpha_{l}}=\alpha_{l}^{(1)} / 2, \rho_{\tau_{l}}=\tau_{l}^{(1)} / 2$, $\rho_{\psi_{z, l}}=0.01 \psi_{z, l}^{(1)}, \rho_{\theta}^{\mathrm{AC}}=\rho_{\varphi}^{\mathrm{AC}}=\pi / 4$, and $\rho_{\theta}^{\mathrm{BS}}=\rho_{\varphi}^{\mathrm{BS}}=\pi / 12$. Note that the maximum value of angle changing during one TI can be approximately calculated as $\frac{\pi}{4} \times T_{\mathrm{TI}} \approx 0.0069^{\circ}$, which is extremely small, so that the assumption about TI is reasonable. For the data-aided channel tracking, $\varepsilon=0.2$ and $\widetilde{K}=K / 2$. Note that the relationship between transmit power $P_{l}$ and large-scale fading gain $G_{l}$ is complementary. Without loss of generality, assume that $P_{l} G_{l}=1$ through the transmit power compensation. Therefore, to facilitate the simulation evaluation, we define $\sigma_{\alpha}^{2} / \sigma_{n}^{2}$ with $\sigma_{n}^{2}$ being the noise variance as the transmitted SNR of UL and DL throughout our simulations.

\section{B. Simulation Results}

First the performance of the initial channel estimation is evaluated using the Root-MSE (RMSE) metric given by $\operatorname{RMSE}_{\boldsymbol{x}}=\sqrt{\mathbb{E}\left(\frac{1}{L}\|\boldsymbol{x}-\widehat{\boldsymbol{x}}\|_{2}^{2}\right)}$, where $\boldsymbol{x} \in \mathbb{R}^{L}$ and $\widehat{\boldsymbol{x}}$ represent the true and the estimated channel parameter vectors, and $[\boldsymbol{x}]_{l}$ comes from the parameters $\theta_{l}^{\mathrm{BS}}, \varphi_{l}^{\mathrm{BS}}, \theta_{l}^{\mathrm{AC}}, \varphi_{l}^{\mathrm{AC}}, \psi_{z, l}$, or $\tau_{l}$. For the angle estimation at the BSs and aircraft, the stateof-the-art channel estimation and tracking schemes [21]-[26], [34] are not suitable for the THz UM-MIMO based aeronautical communication channels with fast time-varying fading characteristics. Hence, we consider the beam sweeping method with severe beam squint effect in IEEE standards 802.11ad [56] as one of the benchmarks, where its sweeping ranges are $\pm 5^{\circ}$ around the corresponding rough angle estimates acquired by BSs and aircraft.

Fig. 11 compares the RMSE performance of the proposed fine angle estimation for $\left\{\theta_{l}^{\mathrm{BS}}, \varphi_{l}^{\mathrm{BS}}\right\}_{l=1}^{L}$ at the initial channel estimation stage, where different processing methods are investigated. In Fig. 11, the labels "no TTDU module" and "ideal TTDU module" indicate the transceiver adopting ideal TTDU module and without considering TTDU module, respectively. The label "conventional scheme" indicates directly applying the conventional TDU-ESPRIT algorithm to estimate angles as those used in existing mmWave systems [37], while $i_{\mathrm{BS}}^{\max }=1$ and $i_{\mathrm{BS}}^{\max }=2$ indicate the maximum iterations in the proposed Algorithm 1. From Fig. 11, it can be seen that the RMSE curves of "proposed algorithm 1 with $i_{\mathrm{BS}}^{\max }=2$ " and "conventional scheme" using "ideal TTDU module" almost overlap, and they are very close to the CRLBs of azimuth and elevation angles at high SNR. The proposed Algorithm 1 just needs $i_{\mathrm{BS}}^{\max }=2$ iterations to achieve the performance upper-bound that uses ideal TTDU module without beam squint effect. If the beam squint effect is not well handled as "conventional scheme" with "no TTDU 


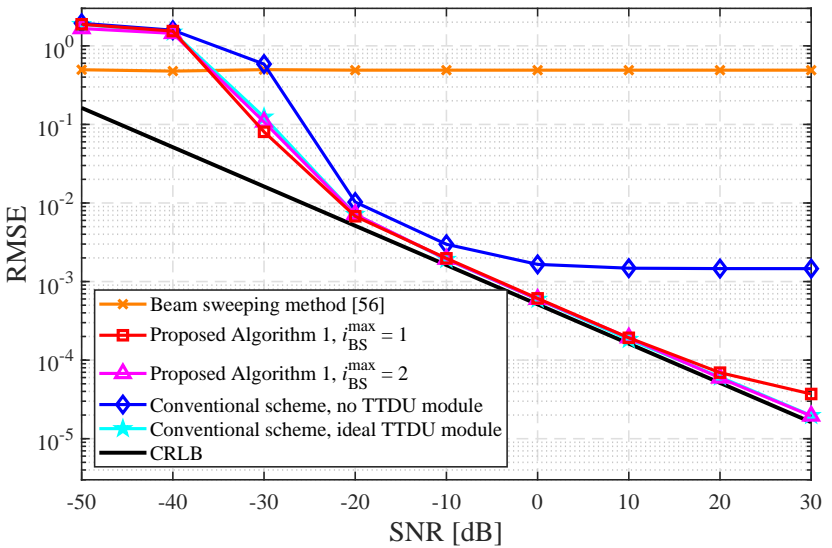

(a) azimuth angle $\theta^{\mathrm{BS}}$

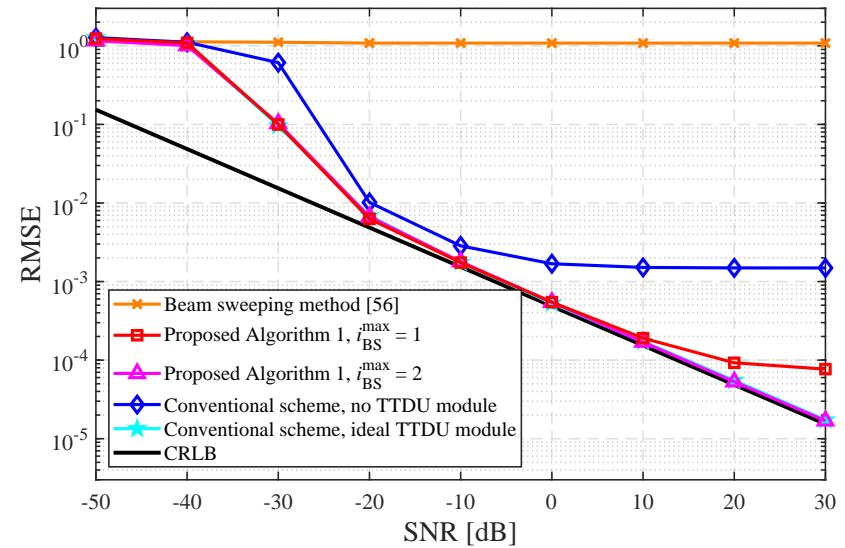

(b) elevation angle $\varphi^{\mathrm{BS}}$

Fig. 11. RMSE comparison of $\left\{\theta^{\mathrm{BS}}, \varphi^{\mathrm{BS}}\right\}$ at the initial angle estimation stage: (a) azimuth angle $\theta^{\mathrm{BS}}$; and (b) elevation angle $\varphi^{\mathrm{BS}}$.

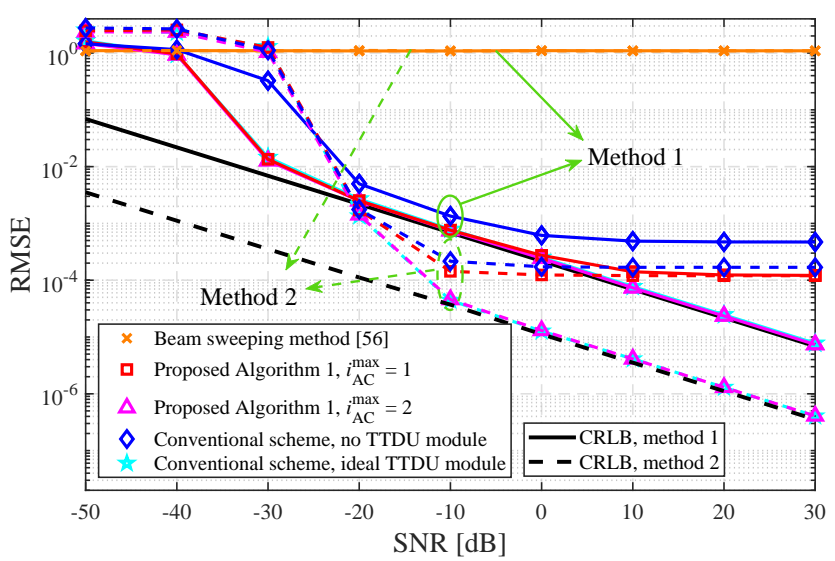

(a) azimuth angle $\theta^{\mathrm{AC}}$

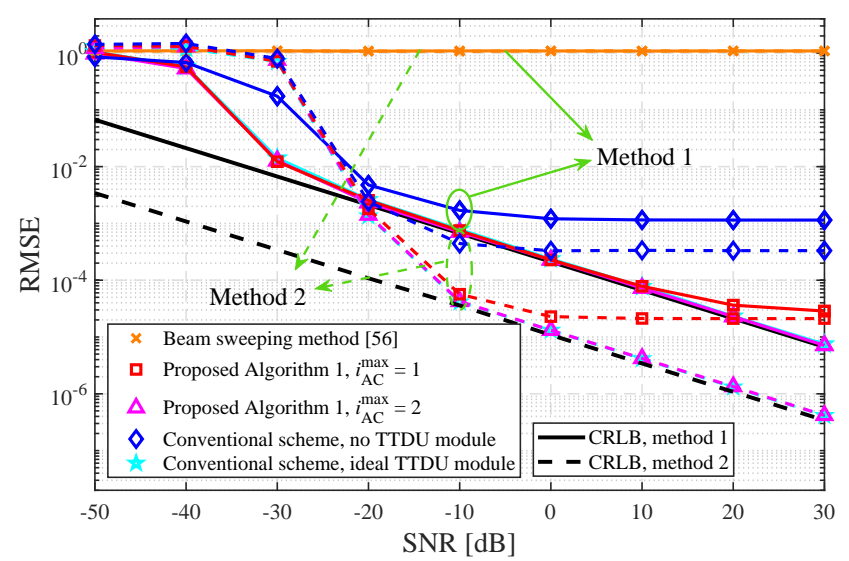

(b) elevation angle $\varphi^{\mathrm{AC}}$

Fig. 12. RMSE comparison of $\left\{\theta^{\mathrm{AC}}, \varphi^{\mathrm{AC}}\right\}$ at the initial angle estimation stage: (a) azimuth angle $\theta^{\mathrm{AC}}$; and (b) elevation angle $\varphi^{\mathrm{AC}}$.

module", its performance of angle estimation will suffer from the obvious RMSE floor at medium-to-high SNR. Note that the angle estimation performance of beam sweeping method is very poor due to the limited training overhead in the fast time-varying channels. Moreover, due to the inaccurately rough angle estimates acquired, "proposed algorithm 1 with $i_{\mathrm{BS}}^{\max }=1$ " only using GTTDU module for compensation at transceiver still suffers from the RMSE floor at high SNR, while "proposed algorithm 1 with $i_{\mathrm{BS}}^{\max }=2$ " can further attenuate this beam squint error by finely compensating the received signal matrix $\boldsymbol{Y}_{\mathrm{UL}, l}$ with the compensation matrix $\tilde{\boldsymbol{Y}}_{\mathrm{UL}, l}^{(1)}$.

Fig. 12 investigates the RMSE performance of the proposed fine angle estimation for $\left\{\theta_{l}^{\mathrm{AC}}, \varphi_{l}^{\mathrm{AC}}\right\}_{l=1}^{L}$ at the initial channel estimation stage. The accurate angle estimation of $\left\{\theta_{l}^{\mathrm{AC}}, \varphi_{l}^{\mathrm{AC}}\right\}_{l=1}^{L}$ relies on the fine estimates of $\left\{\theta_{l}^{\mathrm{BS}}, \varphi_{l}^{\mathrm{BS}}\right\}_{l=1}^{L}$ in Fig. 11. To investigate the impact of the estimated $\left\{\theta_{l}^{\mathrm{BS}}, \varphi_{l}^{\mathrm{BS}}\right\}_{l=1}^{L}$ on the estimation of $\left\{\theta_{l}^{\mathrm{AC}}, \varphi_{l}^{\mathrm{AC}}\right\}_{l=1}^{L}$, we consider "Method 1" and "Method 2". "Method 1" adopts $\left\{\theta_{l}^{\mathrm{BS}}, \varphi_{l}^{\mathrm{BS}}\right\}_{l=1}^{L}$ estimated at BSs for the fixed SNR $=-20 \mathrm{~dB}$, while "Method 2" adopts the $\left\{\theta_{l}^{\mathrm{BS}}, \varphi_{l}^{\mathrm{BS}}\right\}_{l=1}^{L}$ estimated at BSs for the same SNRs with those of the angle estimation at aircraft ${ }^{9}$. From Fig. 12, similar conclusions to those observed for Fig. 11 can be obtained. Moreover, it can be observed that the "Method 2" can obtain more accurate angle estimation than that of "Method 1" when SNR is larger than $-20 \mathrm{~dB}$. For the curves labeled as "proposed algorithm 1 with $i_{\mathrm{AC}}^{\max }=1$ ", "proposed algorithm 1 with $i_{\mathrm{AC}}^{\max }=2$ " and "CRLB", the improvement of RMSE performance are more than $12 \mathrm{~dB}$ when SNR $\geq-10 \mathrm{~dB}$. This is because "Method 2" employs more accurate angles estimated at BSs in high SNR region to obtain the larger beam alignment gain than "Method 1".

Fig. 13 compares the RMSE performance of the proposed fine Doppler estimation for $\left\{\psi_{z, l}\right\}_{l=1}^{L}$ at the initial channel estimation stage with different processing methods, where the angles at BSs and aircraft are estimated at the fixed SNR = $-20 \mathrm{~dB}$. Note that the label "no Doppler squint" denotes the channel model without Doppler squint effect, and the label "proposed algorithm 2 with $i_{\mathrm{do}}^{\max }=0$ " indicates that the TLSESPRIT algorithm is applied directly to $\boldsymbol{Y}_{\mathrm{do}, l}$ for obtaining the estimate $\widehat{\psi}_{z, l}^{(0)}$ in Algorithm 2. From Fig. 13, we observe that the THz UM-MIMO array can provide a large beam alignment

${ }^{9}$ It's worth noting that to ensure the rationality of CRLB at low SNRs for "Method 2", the rough angle estimates $\left\{\widetilde{\theta}_{l}^{\mathrm{BS}}, \widetilde{\varphi}_{l}^{\mathrm{BS}}\right\}_{l=1}^{L}$ rather than the estimated angle $\left\{\widehat{\theta}_{l}^{\mathrm{BS}}, \widehat{\varphi}_{l}^{\mathrm{BS}}\right\}_{l=1}^{L}$ are considered as the beam-aligned angles at BSs when $\mathrm{SNR} \leq-20 \mathrm{~dB}$. 


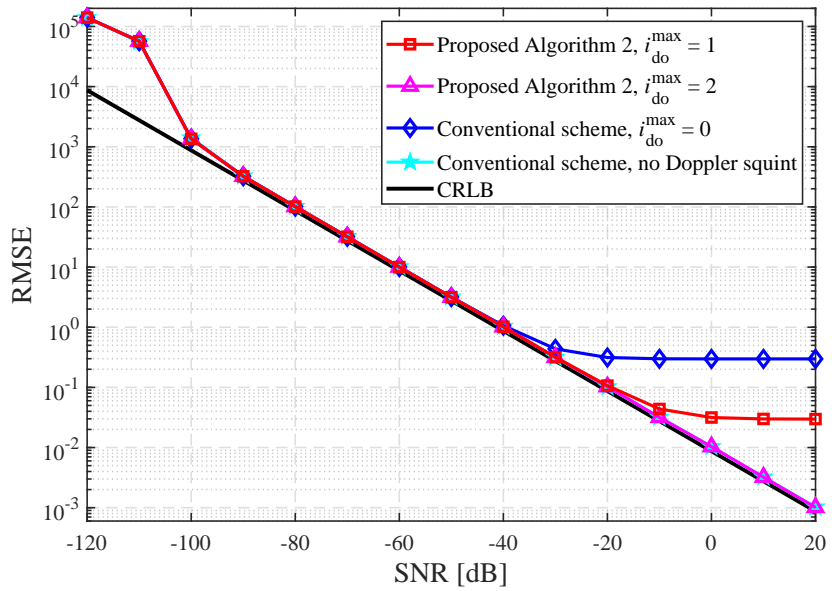

Fig. 13. RMSE comparison of Doppler shift $\psi_{z}$ estimation.

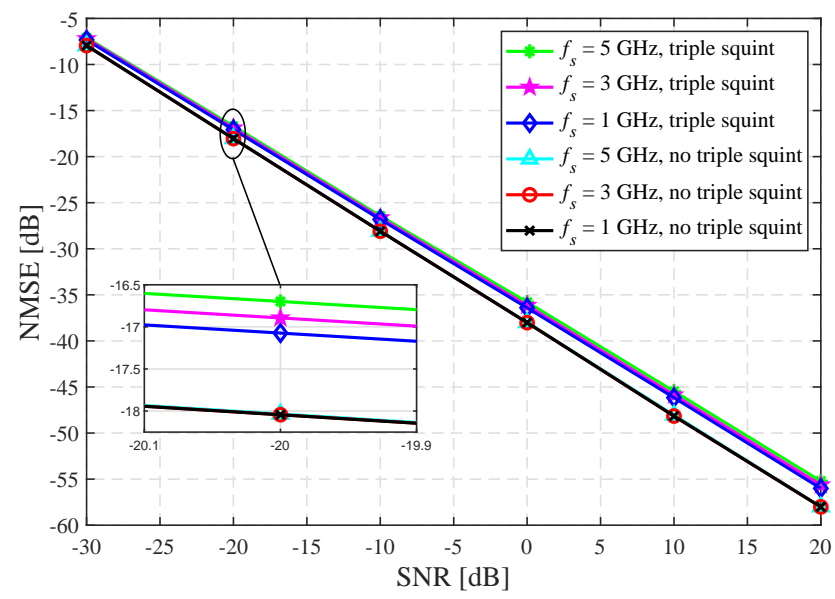

Fig. 15. NMSE comparison with different bandwidths.

gain and greatly improve the receive SNR for Doppler shift estimation, so that the RMSE curves are close to CRLB at very low SNR, even SNR $=-100 \mathrm{~dB}$. Additionally, "proposed algorithm 2 with $i_{\mathrm{do}}^{\max }=0$ " and "proposed algorithm 2 with $i_{\mathrm{do}}^{\max }=1$ " will encounter the RMSE floors at high SNR, while the curve labeled as "proposed algorithm 2 with $i_{\mathrm{do}}^{\max }=2$ " almost overlap with "conventional scheme" with "no beam squint" when SNR $>-100 \mathrm{~dB}$.

Fig. 14 compares the RMSE performance of the proposed path delay estimation for the normalized $\left\{\bar{\tau}_{l}\right\}_{l=1}^{L}$ at the initial channel estimation stage, where the angles and Doppler shifts are estimated at fixed $\mathrm{SNR}=-20 \mathrm{~dB}$ and $\mathrm{SNR}=20 \mathrm{~dB}$, respectively. Note that the labels "triple squint" and "no triple squint" indicate the channel model considering and not considering the practical triple squint effects, respectively. Clearly, when the triple squint effects are considered, the higher angle and Doppler estimation accuracy at SNR $=20 \mathrm{~dB}$ will attenuate the impact of triple squint effects to acquire more accurate path delay estimation than that estimated at

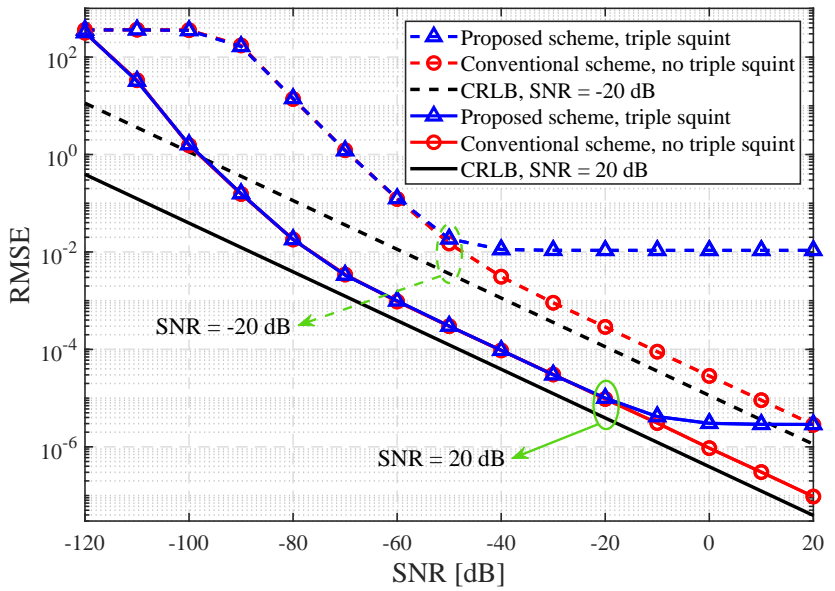

Fig. 14. RMSE comparison of the normalized delay $\bar{\tau}$ estimation.

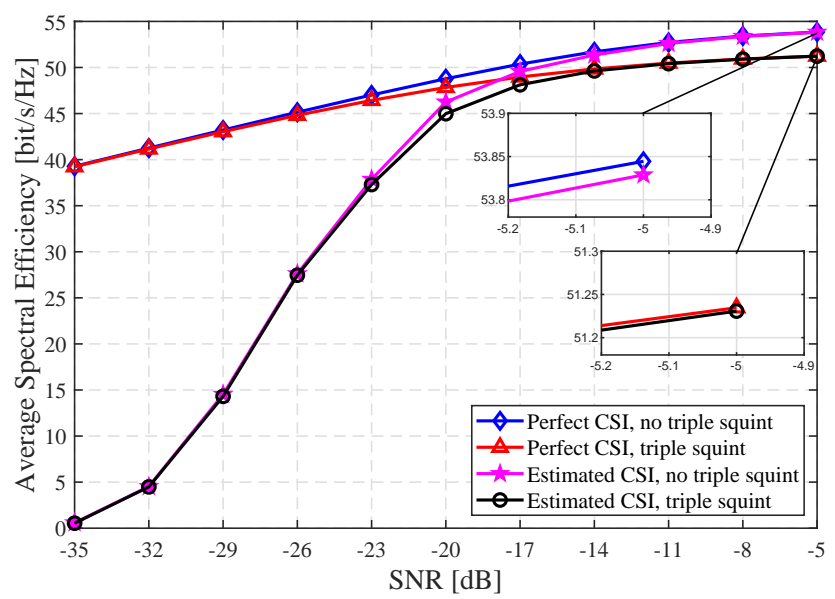

Fig. 16. ASE comparison with different CSI.

$\mathrm{SNR}=-20 \mathrm{~dB}$. Note that the errors of the previously estimated angles $\left\{\widehat{\theta}_{l}^{\mathrm{BS}}, \widehat{\varphi}_{l}^{\mathrm{BS}}, \widehat{\theta}_{l}^{\mathrm{AC}}, \widehat{\varphi}_{l}^{\mathrm{AC}}\right\}_{l=1}^{L}$ and Doppler shifts $\left\{\widehat{\psi}_{z, l}\right\}_{l=1}^{L}$ impact on the estimation of $\left\{\bar{\tau}_{l}\right\}_{l=1}^{L}$, which leads to the RMSE floors of the normalized delay estimation at high SNR.

According to the estimated channel parameters, the Normalized-MSE (NMSE) metric [37] for the initial channel estimation can be expressed as (46) on the bottom of this page. In (46), $\boldsymbol{H}_{\mathrm{DL}, l}^{[2]}[k]$ and $\widehat{\boldsymbol{H}}_{\mathrm{DL}, l}^{[2]}[k]$ denote the DL spatialfrequency channel matrix at the $k$ th subcarrier of the 2nd OFDM symbol (considering the impact of Doppler shifts) in (4) and the reestablished channel matrix based on the estimated channel parameters, respectively. Fig. 15 compares the NMSE performance at the initial channel estimation stage for different system bandwidths $f_{s}=\{1,3,5\}$ GHz. From Fig. 15, we can observe that the channel estimation performance of the proposed solution under triple squint effects is very close to that of the proposed solution without triple squint effects, where the NMSE performance gap between them is about $1 \mathrm{~dB}$

$$
\operatorname{NMSE}_{\boldsymbol{H}_{\mathrm{DL}}^{[2]}}=\mathbb{E}\left(\frac{1}{L} \sum_{l=1}^{L}\left(\sum_{k=1}^{K}\left\|\boldsymbol{H}_{\mathrm{DL}, l}^{[2]}[k]-\widehat{\boldsymbol{H}}_{\mathrm{DL}, l}^{[2]}[k]\right\|_{F}^{2} / \sum_{k=1}^{K}\left\|\boldsymbol{H}_{\mathrm{DL}, l}^{[2]}[k]\right\|_{F}^{2}\right)\right) .
$$






(a)

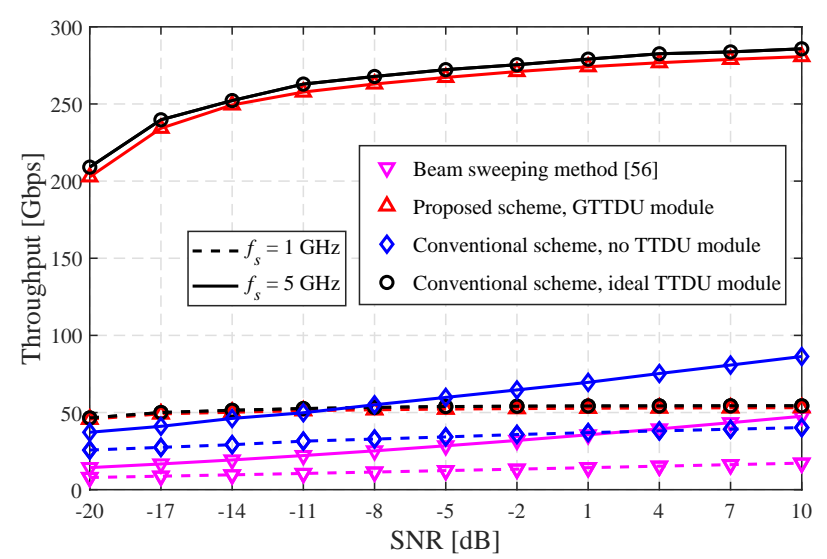

(b)

Fig. 17. Throughput performance comparison of THz UM-MIMO system adopting different TTDU modules: (a) maximum bandwidth is $f_{s}=1 \mathrm{GHz}$ with perfect and the estimated CSI at SNR $=10 \mathrm{~dB}$; and (b) bandwidth $f_{s}=1 \mathrm{GHz}$ and $5 \mathrm{GHz}$ with the estimated CSI.



Fig. 18. Throughput performance comparison of THz UM-MIMO system adopting different dimensions of UPA at $\mathrm{SNR}=10 \mathrm{~dB}$.

at $\mathrm{SNR}=-20 \mathrm{~dB}$. Furthermore, the results of Fig. 15 show that compared with the system bandwidth $f_{s}=1 \mathrm{GHz}$, the NMSE performance of the proposed solution using the larger bandwidth $f_{s}=5 \mathrm{GHz}$ does not deteriorate significantly.

Moreover, we consider the Average Spectral Efficiency (ASE) performance metric [37], [57] at the data transmission stage, defined as ASE = $\sum_{l=1}^{L}\left(\frac{1}{K} \sum_{k=1}^{K} \log _{2}\left(1+\left|h_{l}^{[2]}[k]\right|^{2} /\left|\mathbb{E}\left(z_{l}^{[2]}[k]\right)\right|^{2}\right)\right)$, where $h_{l}^{[2]}[k]$ and $z_{l}^{[2]}[k]$ are the beam-aligned effective channel coefficient and interference plus noise at the $k$ th subcarrier of the 2nd OFDM symbol, respectively. Fig. 16 compares the ASE performance of the proposed solution with different CSI, where the perfect CSI known at both the BSs and aircraft is adopted as the performance upper bound. It can be observed from Fig. 16 that the ASE performance using the estimated CSI almost attains the performance upper bound when SNR $\geq-14 \mathrm{~dB}$ whether or not the triple squint effects are considered. In addition, since the practicable GTTDU module still has residual beam alignment error caused by beam squint effect, the ASE performance gain achieved by our solution with triple squint effects is $2.5[\mathrm{bit} / \mathrm{s} / \mathrm{Hz}]$ lower than the other one at high SNR.
Fig. 17 compares the throughput performance of THz UMMIMO system adopting different TTDU modules, where the transceivers using ideal TTDU module, the proposed GTTDU module, and without TTDU module are considered. Note that $\Delta f$ denotes the frequency spacing between adjacent subcarriers, typically, $\Delta f \approx 0.488$ Megahertz $(\mathrm{MHz})$ for $f_{s}=1 \mathrm{GHz}$ and $K=2048$. In Fig. 17(a), for maximum bandwidth $f_{s}=1 \mathrm{GHz}$, an obvious throughput ceiling can be observed in "beam sweeping method" and conventional scheme with "no TTDU module" as the bandwidth increases, in other words, the severe beam squint effect will restrict the throughput of THz UM-MIMO systems. On the contrary, the throughputs adopting the proposed GTTDU module and ideal TTDU module present a linear growth with the increase of bandwidth. For the estimated CSI at $f_{s}=2048 \Delta f$, the throughput improvements of more than 15 Gigabit per second (Gbps) and 35 Gbps can be acquired by both "ideal TTDU module" and "proposed GTTDU module" compared with the throughput of "no TTDU module" and beam sweeping method in [56], respectively. Furthermore, it can be also observed from Fig. 17(b) that the increase of throughput in the THz UMMIMO system with severe beam squint effect is extremely limited when the bandwidth is increased to $f_{s}=5 \mathrm{GHz}$.

Fig. 18 compares the throughput performance of $\mathrm{THz}$ UM-MIMO system adopting different dimensions of UPA at $\mathrm{SNR}=10 \mathrm{~dB}$, where bandwidth $f_{s}=1 \mathrm{GHz}$ and the same transmit power are considered. From Fig. 18, it can be observed that the usage of regular UPA with size of $16 \times 16$ in the ultra-long-distance $\mathrm{THz}$ aeronautical communications cannot establish an efficient communication link, which causes the degraded throughput performance. Due to the pencil-like beams and less interference, the system throughput will be improved significantly as the dimension of UPA equipped at the transceiver increases. However, the increase of array dimension leads to more obvious beam squint effect, which inhibits the improvement of throughput performance in turn (observed from the curves labeled as "no TTDU module"). For the transceiver equipped with UM-MIMO array of size $256 \times 256$, the throughput adopting the proposed GTTDU module is closed to the throughput of "ideal TTDU mod- 


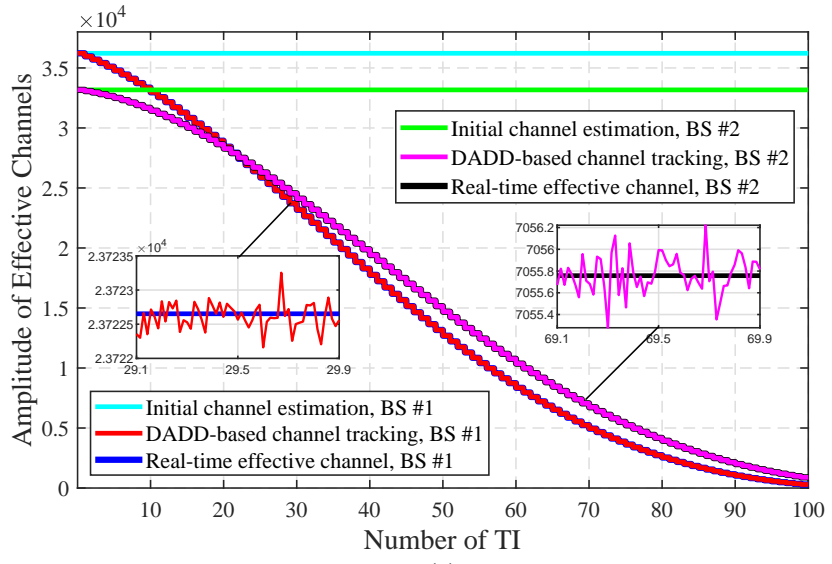

(a)

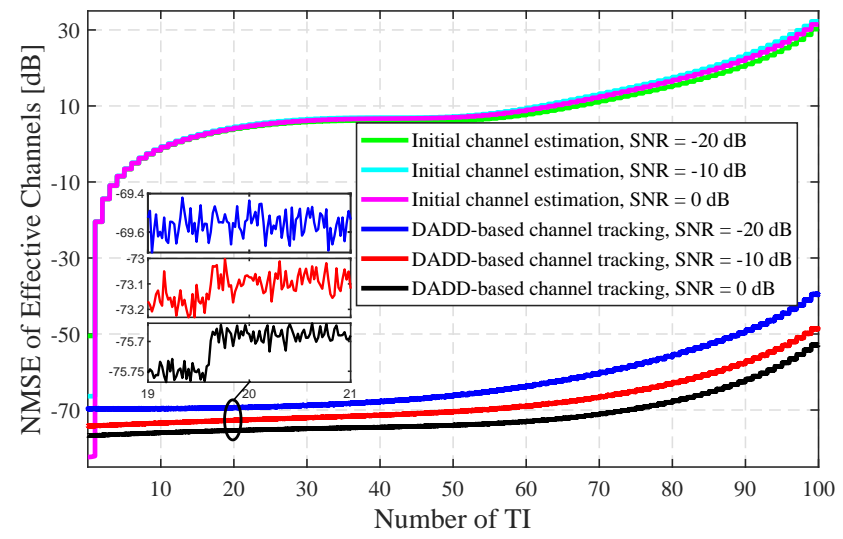

(b)

Fig. 19. Performance comparison of the proposed DADD-based channel tracking: (a) amplitude of effective channels; and (b) NMSE of effective channels.

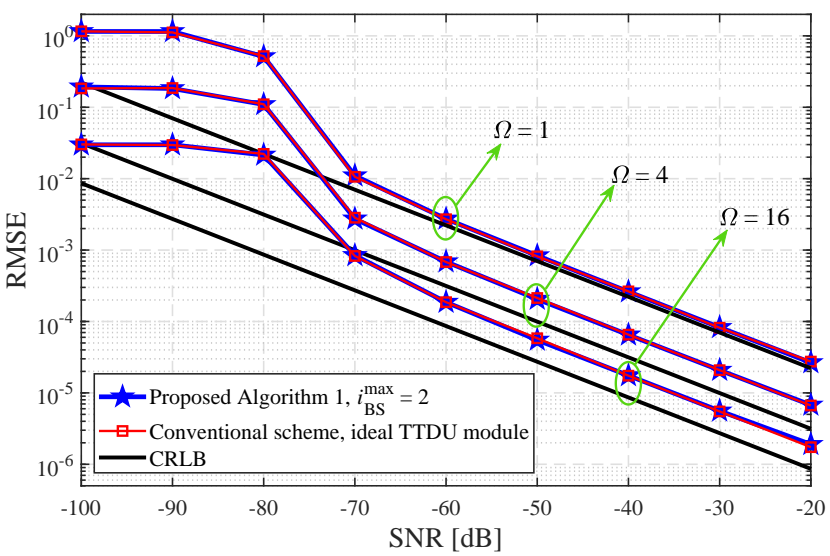

(a) azimuth angle at $\mathrm{BS}: \theta^{\mathrm{BS}}$

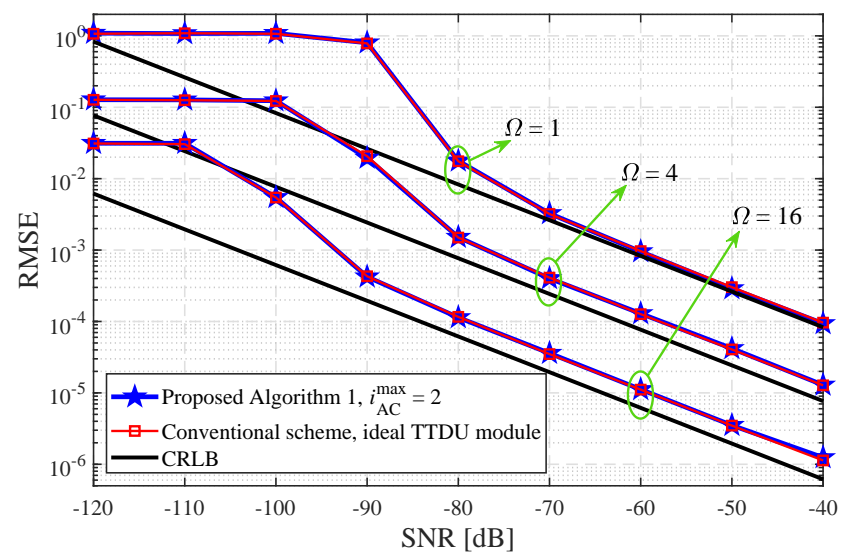

(b) azimuth angle at aircraft: $\theta^{\mathrm{AC}}$

Fig. 20. RMSE performance at pilot-aided angle tracking stage: (a) azimuth angle $\theta^{\mathrm{BS}}$ at BS; and (b) azimuth angle $\theta^{\mathrm{AC}}$ at aircraft.

ule", and it can achieve throughput improvement more than 55 Gbps compared with that of transceiver using UPA of size $16 \times 16$. Therefore, it is necessary to use UM-MIMO array in aeronautical communications to cater for the high data rate requirements of hundreds of users in the cabin.

Next, the performance of the proposed DADD-based channel tracking algorithm is evaluated according to the metrics of effective channels' amplitude and NMSE, where the NMSE of effective channels for the $r$ th OFDM symbol is given by $\operatorname{NMSE}_{\boldsymbol{h}^{[r]}}=\mathbb{E}\left(\frac{1}{L} \sum_{l=1}^{L}\left(\left\|\boldsymbol{h}_{l}^{[r]}-\widehat{\boldsymbol{h}}_{l}^{[r]}\right\|_{2}^{2} /\left\|\boldsymbol{h}_{l}^{[r]}\right\|_{2}^{2}\right)\right)$. For the data-aided channel tracking scheme, Fig. 19 compares the effective channels' amplitude performance (at SNR $=-20 \mathrm{~dB}$ ) and NMSE performance (at SNR $=-20,-10$, and $0 \mathrm{~dB}$ ) for the different numbers of TI. Here, the Turbo coding and QPSK modulation are considered during the data transmission. From Fig. 19(a), we can observe that the amplitude of effective channels decreases rapidly as time goes by, where the proposed DADD-based channel tracking method can track the amplitude changes of true effective channels in real-time. This decreasing amplitudes mean that the gains of beam alignment becomes small. Also observe in Fig. 19(b) that the NMSE performance of the proposed DADD-based channel tracking method slowly worsens as the number of TI increases, while the NMSE of the initial channel estimation without tracking will deteriorate rapidly after several TIs.

Fig. 20 investigates the RMSE performance of the proposed pilot-aided angle tracking scheme against different sparse spacing $\Omega=1, \Omega=4$, and $\Omega=16$. Here the angle tracking at aircraft adopts the angles $\left\{\widehat{\theta}_{l}^{\mathrm{BS}}, \widehat{\varphi}_{l}^{\mathrm{BS}}\right\}_{l=1}^{L}$ estimated at BSs using the fixed $\mathrm{SNR}=-60 \mathrm{~dB}$. Note that the RMSE curves of the elevation angles $\varphi^{\mathrm{BS}}$ and $\varphi^{\mathrm{AC}}$ are omitted due to the similar performance to the azimuth angles. From Fig. 20, it can be observed that the usage of sparse array can significantly improve the accuracy of angle estimation, and these results testify that the improved RMSE performance is consistent with the conclusion in Remark 2, i.e., the proposed solution using the sparse array with sparse spacing $\Omega$ can achieve about $20 \lg \Omega \mathrm{dB}$ performance gain.

\section{CONCLUSIONS}

We have proposed an effective channel estimation and tracking scheme for THz UM-MIMO-based aeronautical communications in SAGIN, which can solve the unique triple delay-beam-Doppler squint effects not considered in the sub$6 \mathrm{GHz}$ or mmWave systems. The proposed solution includes the initial channel estimation, data-aided channel tracking, and pilot-aided channel tracking. Specifically, based on the rough angle estimates acquired from navigation information, 
the initial THz UM-MIMO link can be established, where the delay-beam squint effects at transceiver can be significantly mitigated by employing the proposed GTTDU module. By exploiting the proposed prior-aided iterative angle estimation algorithm, the fine azimuth/elevation angles can be estimated based on the equivalent low-dimensional fully-digital array. These estimated angles can be used not only to achieve a highly accurate beam alignment, but also to refine the GTTDU module at the transceiver for further eliminating the delaybeam squint effects. The Doppler shifts can be subsequently estimated using the proposed prior-aided iterative Doppler shift estimation algorithm. On this basis, path delays and channel gains can be estimated accurately, where Doppler squint effect can be attenuated vastly via fine compensation process. At the data transmission stage, a DADD-based channel tracking algorithm is developed to track the beam-aligned effective channels. When the data-aided channel tracking is invalid, the pilot-aided channel tracking is proposed to reestimate the angles at transceiver using an equivalent fullydigital sparse array, where the angle ambiguity issue derived from sparse array can be addressed based on the previously estimated angles. Finally, the CRLBs of dominant channel parameters and the simulation results evaluate the effectiveness of the proposed solution for THz UM-MIMO-based aeronautical communications.

It is worth mentioning that the proposed solution in this paper still has some improvements in the following aspects. First, the proposed Rotman lens-based GTTDU module of transceiver in Fig. 6 can be further researched. Second, the signal frame structure (e.g., the length of OFDM symbols, CP length) in $\mathrm{THz}$ communications can be also optimized based on the parameter configurations of specific scenarios. Third, some new data-aided channel tracking methods with the lower computational complexity can be considered in Section IV, such as uniformly-spaced pilot interpolation in the frequency domain. Fourth, according to the specific communication scenarios, the transmit power at the transceiver can be also further optimized to improve the spectrum efficiency of systems and reduce the bit error rate.

For future work, our proposed $\mathrm{THz}$ UM-MIMO-based aeronautical communication solution can be also suitable for the long distance communications or backhaul in SAGIN such as the space information network consisting of aircrafts/UAVs, aerial BSs, and LEO/MEO/GEO satellites, or the air-ground communication links between the high-altitude terrestrial stations and the LEO satellite systems. Potential research directions in the THz UM-MIMO-based aeronautical communications include more specific and universal THz UM-MIMO channel modeling under LoS path scenario [9], long-distance air-ground communication scheme design, low-complexity signal transmission and tracking methods for the large bandwidth and high dynamic environment, $\mathrm{THz}$ transceiver design using more practical hardware components (e.g., TTDU module, high-frequency switch [58], and lowenergy antenna array [2]), advanced DSP module design supporting ultra-high data rate with the order of Tbps, modulation and coding design at the physical layer [10], as well as the deployment and power optimization of aerial BSs at the network and transport layer.

\section{APPENDIX A \\ Derivation of DL Channel MATRIX $\boldsymbol{H}_{\mathrm{DL}, l}^{[n]}[k]$}

By taking the Fourier transform of (3) with respect to $\tau$, the frequency response of $\left[\overline{\boldsymbol{H}}_{\mathrm{DL}, l}^{(t)}(\tau)\right]_{n_{\mathrm{AC}}, n_{\mathrm{BS}}}$ is given by

$$
\begin{aligned}
{\left[\overline{\boldsymbol{H}}_{\mathrm{DL}, l}^{(t)}\left(f_{c}\right)\right]_{n_{\mathrm{AC}}, n_{\mathrm{BS}}}=} & \sqrt{G_{l}} \alpha_{l} e^{\mathrm{j} 2 \pi \psi_{l} t} e^{-\mathrm{j} 2 \pi f_{c} \tau_{l}} e^{-\mathrm{j} 2 \pi f_{c} \tau_{l}^{\left[n_{\mathrm{AC}}\right]}} \\
& \times e^{-\mathrm{j} 2 \pi f_{c} \tau_{l}^{\left[n_{\mathrm{BS}}\right]}}
\end{aligned}
$$

where the large-scale fading gain $G_{l}$ can be modeled as $G_{l}=\lambda_{c}^{2} /\left(4 \pi D_{l}\right)^{2}$ based on the free-space path loss of Friis' formula with $D_{l}$ being the communication distance between the aircraft and the lth BS. Considering the large system bandwidth $f_{s}$, the carrier frequency can be expressed as $f_{c}=f_{z}+f$, where $f$ denotes the baseband frequency satisfying $-f_{s} / 2 \leq f \leq f_{s} / 2$ and the wavelength corresponding to the central carrier frequency $f_{z}$ is $\lambda_{z}$. After the down-conversion and focussing on the baseband frequency, we can obtain the $\left(n_{\mathrm{AC}}, n_{\mathrm{BS}}\right)$ th element of the DL baseband channel matrix $\boldsymbol{H}_{\mathrm{DL}, l}^{(t)}(f)$ in the spatial-frequency domain [29], [34], [59], i.e., (48) on the bottom of this page.

Due to the large bandwidth in THz UM-MIMO, the carrier frequencies and wavelengths at different subcarriers are different, so the frequency-dependent Doppler shift at the $k$ th subcarrier is given by $\psi_{l, k}=\psi_{z, l}+\frac{\underline{v}_{l}}{c}\left(\frac{k-1}{K}-\frac{1}{2}\right) f_{s}$ with $\psi_{z, l}=\underline{v}_{l} / \lambda_{z}$. Let the antenna spacing $d=\lambda_{z} / 2$, the baseband frequency response in (48) can be further expressed as the spatial-frequency channel coefficient at the $k$ th subcarrier, i.e., (49) on the bottom of this page. In (49), $\boldsymbol{a}_{\mathrm{AC}}\left(\mu_{l}^{\mathrm{AC}}, \nu_{l}^{\mathrm{AC}}, k\right) \in$ $\mathbb{C}^{N_{\mathrm{AC}}}$ and $\boldsymbol{a}_{\mathrm{BS}}\left(\mu_{l}^{\mathrm{BS}}, \nu_{l}^{\mathrm{BS}}, k\right) \in \mathbb{C}^{N_{\mathrm{BS}}}$ are the array response vectors associated with the $k$ th subcarrier at aircraft and the $l$ th $\mathrm{BS}$, respectively, and $\left[\boldsymbol{a}_{\mathrm{AC}}\left(\mu_{l}^{\mathrm{AC}}, \nu_{l}^{\mathrm{AC}}, k\right)\right]_{n_{\mathrm{AC}}}$ and

$$
\begin{gathered}
{\left[\boldsymbol{H}_{\mathrm{DL}, l}^{(t)}(f)\right]_{n_{\mathrm{AC}}, n_{\mathrm{BS}}}=\sqrt{G_{l}} \alpha_{l} e^{\mathrm{j} 2 \pi \psi_{l} t} e^{-\mathrm{j} 2 \pi f \tau_{l}} e^{\mathrm{j} \frac{2 d}{\lambda_{c}}\left(\left(n_{\mathrm{AC}}^{\mathrm{h}}-1\right) \mu_{l}^{\mathrm{AC}}+\left(n_{\mathrm{AC}}^{\mathrm{v}}-1\right) \nu_{l}^{\mathrm{AC}}\right)} e^{-\mathrm{j} \frac{2 d}{\lambda_{c}}\left(\left(n_{\mathrm{BS}}^{\mathrm{h}}-1\right) \mu_{l}^{\mathrm{BS}}+\left(n_{\mathrm{BS}}^{\mathrm{v}}-1\right) \nu_{l}^{\mathrm{BS}}\right)} .} \\
{\left[\boldsymbol{H}_{\mathrm{DL}, l}^{(t)}[k]\right]_{n_{\mathrm{AC}}, n_{\mathrm{BS}}}=\sqrt{G_{l}} \alpha_{l} e^{\mathrm{j} 2 \pi \psi_{l, k} t} e^{-\mathrm{j} 2 \pi\left(\frac{k-1}{K}-\frac{1}{2}\right) f_{s} \tau_{l}}\left[\boldsymbol{a}_{\mathrm{AC}}\left(\mu_{l}^{\mathrm{AC}}, \nu_{l}^{\mathrm{AC}}, k\right)\right]_{n_{\mathrm{AC}}}\left[\boldsymbol{a}_{\mathrm{BS}}^{*}\left(\mu_{l}^{\mathrm{BS}}, \nu_{l}^{\mathrm{BS}}, k\right)\right]_{n_{\mathrm{BS}}} \cdot} \\
{\left[\boldsymbol{a}_{\mathrm{AC}}\left(\mu_{l}^{\mathrm{AC}}, \nu_{l}^{\mathrm{AC}}, k\right)\right]_{n_{\mathrm{AC}}}=e^{\mathrm{j}\left(\left(n_{\mathrm{AC}}^{\mathrm{h}}-1\right) \mu_{l}^{\mathrm{AC}}+\left(n_{\mathrm{AC}}^{\mathrm{v}}-1\right) \nu_{l}^{\mathrm{AC}}\right)} e^{\mathrm{j}\left(\frac{k-1}{K}-\frac{1}{2}\right) \frac{f_{s}}{f_{z}}\left(\left(n_{\mathrm{AC}}^{\mathrm{h}}-1\right) \mu_{l}^{\mathrm{AC}}+\left(n_{\mathrm{AC}}^{\mathrm{v}}-1\right) \nu_{l}^{\mathrm{AC}}\right)},} \\
{\left[\boldsymbol{a}_{\mathrm{BS}}\left(\mu_{l}^{\mathrm{BS}}, \nu_{l}^{\mathrm{BS}}, k\right)\right]_{n_{\mathrm{BS}}}=e^{\mathrm{j}\left(\left(n_{\mathrm{BS}}^{\mathrm{h}}-1\right) \mu_{l}^{\mathrm{BS}}+\left(n_{\mathrm{BS}}^{\mathrm{v}}-1\right) \nu_{l}^{\mathrm{BS}}\right)} e^{\mathrm{j}\left(\frac{k-1}{K}-\frac{1}{2}\right) \frac{f_{s}}{f_{z}}\left(\left(n_{\mathrm{BS}}^{\mathrm{h}}-1\right) \mu_{l}^{\mathrm{BS}}+\left(n_{\mathrm{BS}}^{\mathrm{v}}-1\right) \nu_{l}^{\mathrm{BS}}\right)} .}
\end{gathered}
$$




$$
\begin{aligned}
\boldsymbol{A}_{\mathrm{DL}, l}[k]= & \boldsymbol{a}_{\mathrm{AC}}\left(\mu_{l}^{\mathrm{AC}}, \nu_{l}^{\mathrm{AC}}, k\right) \boldsymbol{a}_{\mathrm{BS}}^{\mathrm{H}}\left(\mu_{l}^{\mathrm{BS}}, \nu_{l}^{\mathrm{BS}}, k\right) \\
= & \left(\boldsymbol{a}_{\mathrm{AC}}\left(\mu_{l}^{\mathrm{AC}}, \nu_{l}^{\mathrm{AC}}\right) \circ \overline{\boldsymbol{a}}_{\mathrm{AC}}\left(\mu_{l}^{\mathrm{AC}}, \nu_{l}^{\mathrm{AC}}, k\right)\right)\left(\boldsymbol{a}_{\mathrm{BS}}\left(\mu_{l}^{\mathrm{BS}}, \nu_{l}^{\mathrm{BS}}\right) \circ \overline{\boldsymbol{a}}_{\mathrm{BS}}\left(\mu_{l}^{\mathrm{BS}}, \nu_{l}^{\mathrm{BS}}, k\right)\right)^{\mathrm{H}} \\
\stackrel{(a)}{=} & \left(\boldsymbol{a}_{\mathrm{AC}}\left(\mu_{l}^{\mathrm{AC}}, \nu_{l}^{\mathrm{AC}}\right) \boldsymbol{a}_{\mathrm{BS}}^{\mathrm{H}}\left(\mu_{l}^{\mathrm{BS}}, \nu_{l}^{\mathrm{BS}}\right)\right) \circ\left(\overline{\boldsymbol{a}}_{\mathrm{AC}}\left(\mu_{l}^{\mathrm{AC}}, \nu_{l}^{\mathrm{AC}}, k\right) \overline{\boldsymbol{a}}_{\mathrm{BS}}^{\mathrm{H}}\left(\mu_{l}^{\mathrm{BS}}, \nu_{l}^{\mathrm{BS}}, k\right)\right) . \\
\widetilde{\tau}_{l}^{\left[n_{\mathrm{AC}}\right]}= & \left(\left(n_{\mathrm{AC}}^{\mathrm{h}}-1\right) d \sin \left(\widetilde{\theta}_{l}^{\mathrm{AC}}\right) \cos \left(\widetilde{\varphi}_{l}^{\mathrm{AC}}\right)+\left(n_{\mathrm{AC}}^{\mathrm{v}}-1\right) d \sin \left(\widetilde{\varphi}_{l}^{\mathrm{AC}}\right)\right) / c, \\
\widetilde{\tau}_{l}^{\left[n_{\mathrm{BS}}\right]}= & -\left(\left(n_{\mathrm{BS}}^{\mathrm{h}}-1\right) d \sin \left(\widetilde{\theta}_{l}^{\mathrm{BS}}\right) \cos \left(\widetilde{\varphi}_{l}^{\mathrm{BS}}\right)+\left(n_{\mathrm{BS}}^{\mathrm{v}}-1\right) d \sin \left(\widetilde{\varphi}_{l}^{\mathrm{BS}}\right)\right) / c . \\
{\left[\widetilde{\boldsymbol{H}}_{\mathrm{DL}, l}^{(t)}[k]\right]_{n_{\mathrm{AC}}, n_{\mathrm{BS}}}=} & {\left[\overline{\boldsymbol{a}}_{\mathrm{AC}}^{*}\left(\widetilde{\mu}_{l}^{\mathrm{AC}}, \widetilde{\nu}_{l}^{\mathrm{AC}}, k\right)\right]_{n_{\mathrm{AC}}}\left[\boldsymbol{H}_{\mathrm{DL}, l}^{(t)}[k]\right]_{n_{\mathrm{AC}}, n_{\mathrm{BS}}}\left[\overline{\boldsymbol{a}}_{\mathrm{BS}}\left(\widetilde{\mu}_{l}^{\mathrm{BS}}, \widetilde{\nu}_{l}^{\mathrm{BS}}, k\right)\right]_{n_{\mathrm{BS}}} . } \\
\widetilde{\boldsymbol{A}}_{\mathrm{DL}, l}[k]= & \left(\boldsymbol{a}_{\mathrm{AC}}\left(\mu_{l}^{\mathrm{AC}}, \nu_{l}^{\mathrm{AC}}\right) \circ \overline{\boldsymbol{a}}_{\mathrm{AC}}\left(\mu_{l}^{\mathrm{AC}}, \nu_{l}^{\mathrm{AC}}, k\right) \circ \overline{\boldsymbol{a}}_{\mathrm{AC}}^{*}\left(\widetilde{\mu}_{l}^{\mathrm{AC}}, \widetilde{\nu}_{l}^{\mathrm{AC}}, k\right)\right) \\
& \times\left(\boldsymbol{a}_{\mathrm{BS}}\left(\mu_{l}^{\mathrm{BS}}, \nu_{l}^{\mathrm{BS}}\right) \circ \overline{\boldsymbol{a}}_{\mathrm{BS}}\left(\mu_{l}^{\mathrm{BS}}, \nu_{l}^{\mathrm{BS}}, k\right) \circ \overline{\boldsymbol{a}}_{\mathrm{BS}}^{*}\left(\widetilde{\mu}_{l}^{\mathrm{BS}}, \widetilde{\nu}_{l}^{\mathrm{BS}}, k\right)\right)^{\mathrm{H}} \\
= & \boldsymbol{A}_{\mathrm{DL}, l}[k] \circ\left(\overline{\boldsymbol{a}}_{\mathrm{AC}}^{*}\left(\widetilde{\mu}_{l}^{\mathrm{AC}}, \widetilde{\nu}_{l}^{\mathrm{AC}}, k\right) \overline{\boldsymbol{a}}_{\mathrm{BS}}^{\mathrm{T}}\left(\widetilde{\mu}_{l}^{\mathrm{BS}}, \widetilde{\nu}_{l}^{\mathrm{BS}}, k\right)\right) .
\end{aligned}
$$

$\left[\boldsymbol{a}_{\mathrm{BS}}\left(\mu_{l}^{\mathrm{BS}}, \nu_{l}^{\mathrm{BS}}, k\right)\right]_{n_{\mathrm{BS}}}$ can be expressed as (50) and (51), respectively, on the bottom of the previous page.

Taking all $N_{\mathrm{AC}}$ and $N_{\mathrm{BS}}$ antennas of THz UM-MIMO arrays at aircraft and the $l$ th BS into consideration, the complete DL spatial-frequency channel matrix at the $k$ th subcarrier of the $n$th OFDM symbol, i.e., $\boldsymbol{H}_{\mathrm{DL}, l}^{[n]}[k]$ in (2), can be then formulated as

$$
\begin{aligned}
\boldsymbol{H}_{\mathrm{DL}, l}^{[n]}[k]= & \sqrt{G_{l}} \alpha_{l} e^{\mathrm{j} 2 \pi \psi_{l, k}(n-1) T_{\mathrm{sym}}} e^{-\mathrm{j} 2 \pi\left(\frac{k-1}{K}-\frac{1}{2}\right) f_{s} \tau_{l}} \\
& \times \boldsymbol{A}_{\mathrm{DL}, l}[k],
\end{aligned}
$$

where the DL array response matrix $\boldsymbol{A}_{\mathrm{DL}, l}[k] \in \mathbb{C}^{N_{\mathrm{AC}} \times N_{\mathrm{BS}}}$ associated with the array response vectors at aircraft and the $l$ th BS is given by (53) on the top of this page. In (53), we have used the identity $(\boldsymbol{a} \circ \boldsymbol{b})(\boldsymbol{c} \circ \boldsymbol{d})^{\mathrm{H}}=\left(\boldsymbol{a} \boldsymbol{c}^{\mathrm{H}}\right) \circ\left(\boldsymbol{b} \boldsymbol{d}^{\mathrm{H}}\right)[60]$ in equation $(a)$.

\section{APPENDIX B}

\section{PROOF OF LEMMA 1}

After compensating the antenna transmission delay via the ideal TTDU module, the compensated $\left(n_{\mathrm{AC}}, n_{\mathrm{BS}}\right)$ th element of DL spatial-delay domain passband channel matrix $\overline{\boldsymbol{H}}_{\mathrm{DL}, l}^{(t)}(\tau)$ in $(3)$, denoted by $\left[\widetilde{\overline{\boldsymbol{H}}}_{\mathrm{DL}, l}^{(t)}(\tau)\right]_{n_{\mathrm{AC}}, n_{\mathrm{BS}}}$, can be expressed as

$$
\begin{aligned}
\left.\widetilde{\widetilde{\boldsymbol{H}}}_{\mathrm{DL}, l}^{(t)}(\tau)\right]_{n_{\mathrm{AC}}, n_{\mathrm{BS}}}= & \delta\left(\tau-\widetilde{\tau}_{l}^{\left[n_{\mathrm{AC}}\right]}\right) \circledast\left[\overline{\boldsymbol{H}}_{\mathrm{DL}, l}^{(t)}(\tau)\right]_{n_{\mathrm{AC}}, n_{\mathrm{BS}}} \\
& \circledast \delta\left(\tau-\widetilde{\tau}_{l}^{\left[n_{\mathrm{BS}}\right]}\right),
\end{aligned}
$$

where $\circledast$ represent the linear convolution operation, and $\widetilde{\tau}_{l}^{\left[n_{\mathrm{AC}}\right]}$ and $\widetilde{\tau}_{l}^{\left[n_{\mathrm{BS}}\right]}$ are the compensated transmission delays yielded by TTDUs at aircraft and BSs, respectively, denoted by (55) and (56), respectively, on the top of this page. Similar to (48), by taking the Fourier transform of (54) and the down-conversion, the baseband frequency domain response of $\left[\widetilde{\overline{\boldsymbol{H}}}_{\mathrm{DL}, l}^{(t)}(\tau)\right]_{n_{\mathrm{AC}}, n_{\mathrm{BS}}}$ is given by

$$
\begin{aligned}
{\left[\widetilde{\boldsymbol{H}}_{\mathrm{DL}, l}^{(t)}(f)\right]_{n_{\mathrm{AC}}, n_{\mathrm{BS}}}=} & e^{\left.-\mathrm{j} 2 \pi f \widetilde{\tau}_{l}^{[n} \mathrm{AC}\right]}\left[\boldsymbol{H}_{\mathrm{DL}, l}^{(t)}(f)\right]_{n_{\mathrm{AC}}, n_{\mathrm{BS}}} \\
& \times e^{-\mathrm{j} 2 \pi f \widetilde{\tau}_{l}^{\left.n n_{\mathrm{BS}}\right]}} .
\end{aligned}
$$

The spatial-frequency channel coefficient at the $k$ th subcarrier $\left[\widetilde{\boldsymbol{H}}_{\mathrm{DL}, l}^{(t)}[k]\right]_{n_{\mathrm{AC}}, n_{\mathrm{BS}}}$ can be then written as (58) on the top of this page. Finally, by collecting all $N_{\mathrm{AC}}$ and $N_{\mathrm{BS}}$ antennas of THz UM-MIMO arrays at aircraft and the $l$ th BS, the compensated DL spatial-frequency channel matrix at the $k$ th subcarrier of the $n$th OFDM symbol, i.e., $\widetilde{\boldsymbol{H}}_{\mathrm{DL}, l}^{[n]}[k]$ in (12), can be formulated as

$$
\begin{aligned}
\widetilde{\boldsymbol{H}}_{\mathrm{DL}, l}^{[n]}[k]= & \sqrt{G_{l}} \alpha_{l} e^{\mathrm{j} 2 \pi \psi_{l, k}(n-1) T_{\mathrm{sym}}} e^{-\mathrm{j} 2 \pi\left(\frac{k-1}{K}-\frac{1}{2}\right) f_{s} \tau_{l}} \\
& \times \widetilde{\boldsymbol{A}}_{\mathrm{DL}, l}[k],
\end{aligned}
$$

where the compensated DL array response matrix $\widetilde{\boldsymbol{A}}_{\mathrm{DL}, l}[k]$ is given by (60) on the top of this page.

The proof of Lemma 1 is completed.

\section{REFERENCES}

[1] P. Yang, Y. Xiao, M. Xiao, and S. Li, "6G wireless communications: Vision and potential techniques," IEEE Netw., vol. 33, no. 4, pp. 70-75, Jul. 2019.

[2] I. F. Akyildiz, J. M. Jornet, and C. Han, "Terahertz band: Next frontier for wireless communications," Phys. Commun., vol. 12, pp. 16-32, Sept. 2014.

[3] C. Han, A. O. Bicen, and I. F. Akyildiz, "Multi-ray channel modeling and wideband characterization for wireless communications in the terahertz band," IEEE Trans. Wireless Commun., vol. 14, no. 5, pp. $2402-$ 2412, May 2015.

[4] I. F. Akyildiz, C. Han, and S. Nie, "Combating the distance problem in the millimeter wave and terahertz frequency bands," IEEE Commun. Mag., vol. 56, no. 6, pp. 102-108, Jun. 2018.

[5] H. Sarieddeen, M.-S. Alouini, and T. Y. Al-Naffouri, "Terahertz-band ultra-massive spatial modulation MIMO,” IEEE J. Sel. Areas Commun., vol. 37, no. 9, pp. 2040-2052, Sept. 2019.

[6] I. F. Akyildiz and J. M. Jornet, "Realizing ultra-massive MIMO (1024× 1024) communication in the (0.06-10) terahertz band," Nano Commun. Netw., vol. 8, pp. 46-54, Jun. 2016.

[7] I. F. Akyildiz, J. M. Jornet, and S. Nie, "A new CubeSat design with reconfigurable multi-band radios for dynamic spectrum satellite communication networks," Ad Hoc Netw., vol. 86, pp. 166-178, Apr. 2019.

[8] I. F. Akyildiz, A. Kak, and S. Nie, "6G and beyond: The future of wireless communications systems," IEEE Access, vol. 8, pp. 133995 134030, Jul. 2020.

[9] C. Han and Y. Chen, "Propagation modeling for wireless communications in the terahertz band," IEEE Commun. Mag., vol. 56, no. 6, pp. 96-101, Jun. 2018. 
[10] I. F. Akyildiz, J. M. Jornet, and C. Han, "TeraNets: Ultra-broadband communication networks in the terahertz band," IEEE Wireless Commun., vol. 21, no. 4, pp. 130-135, Aug. 2014.

[11] C. Han, A. O. Bicen, and I. F. Akyildiz, "Multi-wideband waveform design for distance-adaptive wireless communications in the terahertz band," IEEE Trans. Signal Process., vol. 64, no. 4, pp. 910-922, Feb. 2016.

[12] C. Han and I. F. Akyildiz, "Distance-aware bandwidth-adaptive resource allocation for wireless systems in the terahertz band," IEEE Trans. THz Sci. Technol., vol. 6, no. 4, pp. 541-553, Jul. 2016.

[13] X. He and X. Xu, "Physics-based prediction of atmospheric transfer characteristics at terahertz frequencies," IEEE Trans. Antennas Propag., vol. 67, no. 4, pp. 2136-2141, Apr. 2019.

[14] H. Elayan, O. Amin, B. Shihada, R. M. Shubair, and M.-S. Alouini, "Terahertz band: The last piece of RF spectrum puzzle for communication systems," IEEE Open J. Commun. Society, vol. 1, pp. 1-32, Nov. 2019.

[15] A. Saeed, O. Gurbuz, and M. A. Akkas, "Terahertz communications at various atmospheric altitudes," Phys. Commun., vol. 41, pp. 1-15, Aug. 2020.

[16] J. Liu, Y. Shi, Z. M. Fadlullah, and N. Kato, "Space-air-ground integrated network: A survey," IEEE Commun. Surveys Tuts., vol. 20, no. 4, pp. 2714-2741, 4th Quart., 2018.

[17] X. Huang, J. A. Zhang, R. P. Liu, Y. J. Guo, and L. Hanzo, "Airplaneaided integrated networking for $6 \mathrm{G}$ wireless: Will it work?" IEEE Veh. Technol. Mag., vol. 14, no. 3, pp. 84-91, Sept. 2019.

[18] J. Zhang et al., "Regularized zero-forcing precoding-aided adaptive coding and modulation for large-scale antenna array-based air-to-air communications," IEEE J. Sel. Areas Commun., vol. 36, no. 9, pp. 20872103, Sept. 2018.

[19] J. Zhang, T. Chen, S. Zhong, J. Wang, W. Zhang, X. Zuo, R. G. Maunder, and L. Hanzo, "Aeronautical ad hoc networking for the internet-abovethe-clouds," Proc. IEEE, vol. 107, no. 5, pp. 868-911, May 2019.

[20] D. Ogbe, D. J. Love, M. Rebholz, and T. P. Bidigare, "Efficient channel estimation for aerial wireless communications," IEEE Trans. Aerosp. Electron. Syst., vol. 55, no. 6, pp. 2774-2785, Dec. 2019.

[21] Z. Gong, F. Jiang, and C. Li, "Angle domain channel tracking with large antenna array for high mobility V2I millimeter wave communications," IEEE J. Sel. Topics Signal Process., vol. 13, no. 5, pp. 1077-1089, Sept. 2019.

[22] J. Ma, S. Zhang, H. Li, F. Gao, and S. Jin, "Sparse bayesian learning for the time-varying massive MIMO channels: Acquisition and tracking," IEEE Trans. Commun., vol. 67, no. 3, pp. 1925-1938, Mar. 2019.

[23] M. Li, S. Zhang, N. Zhao, W. Zhang, and X. Wang, "Time-varying massive MIMO channel estimation: Capturing, reconstruction, and restoration," IEEE Trans. Commun., vol. 67, no. 11, pp. 7558-7572, Nov. 2019.

[24] Q. Qin, L. Gui, P. Cheng, and B. Gong, "Time-varying channel estimation for millimeter wave multiuser MIMO systems," IEEE Trans. Veh. Technol., vol. 67, no. 10, pp. 9435-9448, Oct. 2018.

[25] L. Cheng, G. Yue, X. Xiong, Y. Liang, and S. Li, "Tensor decompositionaided time-varying channel estimation for millimeter wave MIMO systems," IEEE Wireless Commun. Lett., vol. 8, no. 4, pp. 1216-1219, Aug. 2019.

[26] X. Gao, L. Dai, Y. Zhang, T. Xie, X. Dai, and Z. Wang, "Fast channel tracking for terahertz beamspace massive MIMO systems," IEEE Trans. Veh. Technol., vol. 66, no. 7, pp. 5689-5696, Jul. 2017.

[27] B. Peng and T. Kürner, "Three-dimensional angle of arrival estimation in dynamic indoor terahertz channels using a forward-backward algorithm," IEEE Trans. Veh. Technol., vol. 66, no. 5, pp. 3798-3811, May 2017.

[28] B. Wang, F. Gao, S. Jin, H. Lin, G. Y. Li, S. Sun, and T. S. Rappaport, "Spatial-wideband effect in massive MIMO with application in mmWave systems," IEEE Commun. Mag., vol. 56, no. 12, pp. 134-141, Dec. 2018.

[29] B. Wang, F. Gao, S. Jin, H. Lin, and G. Y. Li, "Spatial- and frequencywideband effects in millimeter-wave massive MIMO systems," IEEE Trans. Signal Process., vol. 66, no. 13, pp. 3393-3406, Jul. 2018.

[30] V. Krozer et al., "Terahertz imaging systems with aperture synthesis techniques," IEEE Trans. Microw. Theory Techn., vol. 58, no. 7, pp. 2027-2039, Jul. 2010.

[31] K. Venugopal, N. G.-Prelcic, and R. W. Heath, "Optimal frequencyflat precoding for frequency-selective millimeter wave channels," IEEE Trans. Wireless Commun., vol. 18, no. 11, pp. 5098-5112, Nov. 2019.

[32] Y. Chen, Y. Xiong, D. Chen, T. Jiang, S. X. Ng, and L. Hanzo, "Hybrid precoding for wideband millimeter wave MIMO systems in the face of beam squint," IEEE Trans. Wireless Commun., doi: 10.1109/TWC.2020.3036945.
[33] M. Jian, F. Gao, Z. Tian, S. Jin, and S. Ma, "Angle-domain aided UL/DL channel estimation for wideband mmWave massive MIMO systems with beam squint," IEEE Trans. Wireless Commun., vol. 18, no. 7, pp. 35153527, Jul. 2019.

[34] B. Wang, M. Jian, F. Gao, G. Y. Li, and H. Lin, "Beam squint and channel estimation for wideband mmWave massive MIMO-OFDM systems," IEEE Trans. Signal Process., vol. 67, no. 23, pp. 5893-5908, Dec. 2019.

[35] M. Wang, F. Gao, N. Shlezinger, M. F. Flanagan, and Y. C. Eldar, "A block sparsity based estimator for mmWave massive MIMO channels with beam squint," IEEE Trans. Signal Process., vol. 68, pp. 49-64, Nov. 2020.

[36] M. Haardt and J. A. Nossek, "Simultaneous Schur decomposition of several nonsymmetric matrices to achieve automatic pairing in multidimensional harmonic retrieval problems," IEEE Trans. Signal Process., vol. 46, no. 1, pp. 161-169, Jan. 1998.

[37] A. Liao, Z. Gao, H. Wang, S. Chen, M.-S. Alouini, and H. Yin, "Closedloop sparse channel estimation for wideband millimeter-wave fulldimensional MIMO systems," IEEE Trans. Commun., vol. 67, no. 12, pp. 8329-8345, Dec. 2019 .

[38] C. Han and I. F. Akyildiz, "Three-dimensional End-to-End modeling and analysis for graphene-enabled terahertz band communications," IEEE Trans. Veh. Technol., vol. 66, no. 7, pp. 5626-5634, Jul. 2017.

[39] J. M. Jornet and I. F. Akyildiz, "Channel modeling and capacity analysis for electromagnetic wireless nanonetworks in the terahertz band," IEEE Trans. Wireless Commun., vol. 10, no. 10, pp. 3211-3221, Oct. 2011.

[40] H. Hashemi, T. Chu, and J. Roderick, "Integrated true-time-delay based ultra-wideband array processing," IEEE Commun. Mag., vol. 46, no. 9, pp. 162-172, Sept. 2008.

[41] C. Lin, G. Y. Li, and L. Wang, "Subarray-based coordinated beamforming training for mmWave and sub-THz communications," IEEE J. Sel. Areas Commun., vol. 35, no. 9, pp. 2115-2126, Sept. 2017.

[42] C. Han, J. M. Jornet, and I. F. Akyildiz, "Ultra-massive MIMO channel modeling for graphene-enabled terahertz-band communications," in Proc. IEEE Veh. Technol. Conf. (VTC Spring), Porto, PT, 2018, pp. 1-5.

[43] D. P.-López, E. Sánchez, and J. Capmany, "Programmable true time delay lines using integrated waveguide meshes," J. Lightwave Technol., vol. 36, no. 19 , pp. $4591-4601$, Oct. 2018

[44] F. Lin and H. Deng, "Continuously tunable true-time-delay phase shifter based on transmission lines with simultaneously reconfigurable impedance and phase constant," IEEE Trans. Microw. Theory Techn., vol. 67, no. 12, pp. 4714-4723, Dec. 2019.

[45] L. Yan, C. Han, and J. Yuan, "A dynamic array-of-subarrays architecture and hybrid precoding algorithms for terahertz wireless communications," IEEE J. Sel. Areas Commun., vol. 38, no. 9, pp. 2041-2056, Sept. 2020.

[46] O. Kodheli et al., "Satellite communications in the new space era: A survey and future challenges," IEEE Commun. Surveys Tuts., doi: 10.1109/COMST.2020.3028247.

[47] R. Rotman, M. Tur, and L. Yaron, "True time delay in phased arrays," Proc. IEEE, vol. 104, no. 3, pp. 504-518, Mar. 2016.

[48] Y. Gao, M. Khaliel, F. Zheng, and T. Kaiser, "Rotman lens based hybrid analog-digital beamforming in massive MIMO systems: Array architectures, beam selection algorithms and experiments," IEEE Trans. Veh. Technol., vol. 66, no. 10, pp. 9134-9148, Oct. 2017.

[49] X. Wang, A. Akbarzadeh, L. Zou, and C. Caloz, "Flexible-resolution, arbitrary-input, and tunable Rotman lens spectrum decomposer," IEEE Trans. Antennas Propag., vol. 66, no. 8, pp. 3936-3947, Aug. 2018.

[50] R. Roy and T. Kailath, "ESPRIT-estimation of signal parameters via rotational invariance techniques," IEEE Trans. Acoust., Speech, Signal Process., vol. 37, no. 7, pp. 984-995, Jul. 1989.

[51] D. Tse and P. Viswanath, Fundamentals of Wireless Communication. New York, NY, USA: The Cambridge Univ. Press, 2005.

[52] S. Chuang, W. Wu, and Y. Liu, "High-resolution AoA estimation for hybrid antenna arrays," IEEE Trans. Antennas Propag., vol. 63, no. 7, pp. 2955-2968, Jul. 2015.

[53] P. Stoica and A. Nehorai, "MUSIC, maximum likelihood, and CramerRao bound," IEEE Trans. Acoust., Speech, Signal Process., vol. 37, no. 5, pp. 720-741, May 1989.

[54] M. C. Vanderveen, A.-J. Van der Veen, and A. Paulraj, "Estimation of multipath parameters in wireless communications," IEEE Trans. Signal Process., vol. 46, no. 3, pp. 682-690, Mar. 1998.

[55] S. M. Kay, Fundamentals of Statistical Signal Processing: Estimation Theory. Engle-wood Cliffs, NJ, USA: Prentice-Hall, 1993.

[56] Wireless LAN Medium Access Control (MAC) and Physical Layer (PHY) Specifications. Amendment 3: Enhancements for Very High Throughput in the $60 \mathrm{GHz}$ Band, IEEE Std. 802.11ad, 2012. 
[57] Z. Wang, M. Li, X. Tian, and Q. Liu, "Iterative hybrid precoder and combiner design for mmWave multiuser MIMO systems," IEEE Commun. Lett., vol. 21, no. 7, pp. 1581-1584, Jul. 2017.

[58] H. Ghaleb, P. V. Testa, S. Schumann, C. Carta, and F. Ellinger, "A 160-GHz switched injection-locked oscillator for phase and amplitude regenerative sampling," IEEE Microw. Wireless Compon. Lett., vol. 27, no. 9 , pp. 821-823, Sept. 2017

[59] X. Lin, S. Wu, L. Kuang, Z. Ni, X. Meng, and C. Jiang, "Estimation of sparse massive MIMO-OFDM channels with approximately common support," IEEE Commun. Lett., vol. 21, no. 5, pp. 1179-1182, May 2017.

[60] S. Liu and G. Trenkler, "Hadamard, Khatri-Rao, Kronecker and other matrix products," Int. J. Inf. Syst. Sci., vol. 4, no. 1, pp. 160-177, 2008. 